SLAC-R-771

August 2005

\title{
ATF2 Proposal
}

\author{
ATF2 Collaboration
}




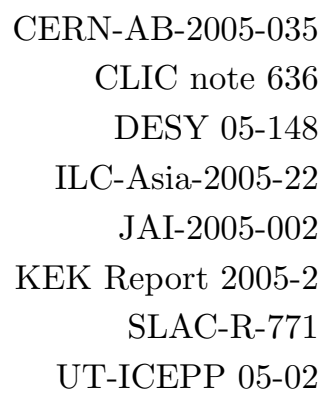

\section{ATF2 Proposal}

\section{ATF2 Collaboration}

August 11, 2005 



\section{Foreword}

A decade of dedicated R\&D at KEK, DESY, CERN, SLAC and other laboratories were crucial to the successful development of the concepts for a linear collider and for demonstrating that the technical goals are achievable. We are now entering the global design phase for the ILC, and test facilities, demonstration experiments and fundamental R\&D will continue to be very important to helping us develop the best possible ILC design, and one that employs forward looking technology.

The ATF2 builds on the considerable investment, success and strong team that were responsible for the ATF. The new features provided by ATF2 will enable us to embark on a program to test the very demanding beam delivery requirements for the ILC. In addition, this project has the feature that it is being planned and executed internationally. Therefore, it represents a useful testing ground for managing and executing a complex international accelerator project.

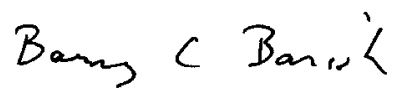

Barry Barish

GDE Director 

Boris Ivanovich Grishanov, Pavel Logachev, Fedor Podgorny, Valery Telnov (BINP SB RAS, Novosibirsk)

Deepa Angal-Kalinin, Robert Appleby, James Jones, Alexander Kalinin (CCLRC/DL/ASTeC,Daresbury, Warrington, Cheshire)

Olivier Napoly, Jacques Payet (CEA/DSM/DAPNIA, Gif-sur-Yvette)

Hans-Heinrich Braun, Daniel Schulte, Frank Zimmermann (CERN, Geneva)

Roger Barlow, Ian Bailey, Leo Jenner, Roger Jones, German Kourevlev (Cockcroft Institute, Daresbury, Warrington, Cheshire)

Nick Walker (DESY, Hamburg)

Tohru Takahashi (Hiroshima University, Higashi-Hiroshima)

Jie Gao, Weibin Liu, Guo-Xi Pei, Jiu-Qing Wang (IHEP, Beijing)

Nicolas Delerue, Sudhir Dixit, David Howell, Armin Reichold, David Urner (John Adams Institute at Oxford University)

Alessio Bosco, Ilya Agapov, Grahame A. Blair ${ }^{1}$, Gary Boorman, John Carter, Chafik Driouichi, Michael Price

(John Adams Institute at Royal Holloway, Univ. of London)

Sakae Araki, Hitoshi Hayano, Yasuo Higashi, Yosuke Honda, Ken-ichi Kanazawa, Kiyoshi Kubo, Tatsuya Kume, Masao Kuriki, Shigeru Kuroda, Mika Masuzawa, Takashi Naito, Toshiyuki Okugi, Ryuhei Sugahara, Toshiaki Tauchi, ${ }^{1}$ Nobuhiro Terunuma, Nobu Toge, Junji Urakawa, Vladimir Vogel, Hiroshi Yamaoka, Kaoru Yokoya (KEK, Ibaraki)

Yoshihisa Iwashita, Takanori Mihara

(Kyoto ICR, Uji, Kyoto)

Philip Bambade

(LAL, Orsay)

Andy Wolski

(LBL, Berkeley, California)

Jeff Gronberg

(LLNL, Livermore, California) 
Stewart Takashi Boogert, Alexey Liapine, Stephen Malton, David J. Miller, Matthew Wing (University College London, London)

Masayuki Kumada

(NIRS, Chiba-shi)

Samuel Danagoulian, Sekazi Mtingwa

(North Carolina A $B$ T State University, North Carolina)

Eric Torrence

(University of Oregon, Eugene, Oregon)

Jinhyuk Choi, Jung-Yun Huang, Heung Sik Kang, Eun-San Kim, Seunghwan Kim, In Soo Ko (Pohang Accelerator Laboratory)

Philip Burrows, Glenn Christian, Christine Clarke, Anthony Hartin, Hamid Dabiri Khah, Stephen Molloy, Glen White (Queen Mary University of London, London)

Karl Bane, Axel Brachmann, Thomas Himel, Thomas Markiewicz, Janice Nelson, Yuri Nosochkov, Nan Phinney, Mauro Torino Francesco Pivi, Tor Raubenheimer, Marc Ross, Robert Ruland, Andrei Seryi ${ }^{1}$, Cherrill M. Spencer, Peter Tenenbaum, Mark Woodley (SLAC, Menlo Park, California)

Sachio Komamiya, Tomoyuki Sanuki ${ }^{1}$, Taikan Suehara

(University of Tokyo, Tokyo)

${ }^{1}$ The Editorial Board 


\section{Contents}

1 Introduction \& Executive Summary $\quad 3$

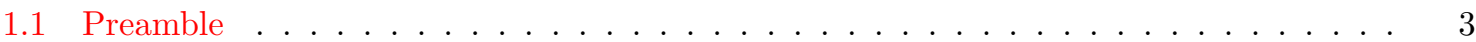

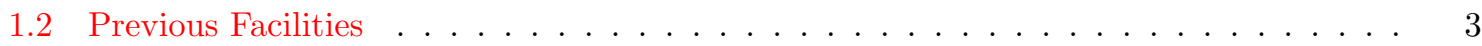

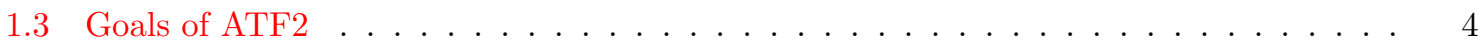

1.4 Requirements on the ATF Beam . . . . . . . . . . . . . . . . . . . 5

1.5 Comparison with ILC FFS . . . . . . . . . . . . . . . . . . . 5

1.6 Scope, Timeline and Budget . . . . . . . . . . . . . . . . . . . 6

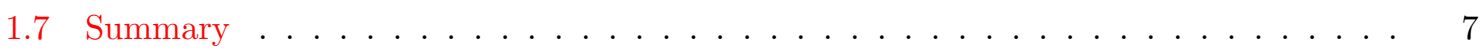

2 Overview of the ATF2 project $\quad 9$

$\begin{array}{llr}3 & \text { Optics } & 11\end{array}$

3.1 ATF2 FF optics and comparison with the ILC-FF . . . . . . . . . . . . . . 11

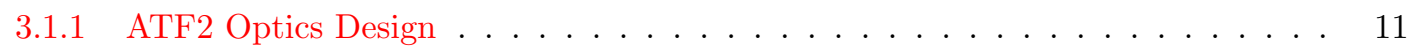

3.1 .2 Proposed optics designs . . . . . . . . . . . . . . . . 12

3.1.3 Bandwidth and tracking results . . . . . . . . . . . . . . . 14

3.1.4 Sensitivity to errors in ATF2 and ILC-FF . . . . . . . . . . . . 15

3.2 Tolerances and tuneability . . . . . . . . . . . . . . . . . . . . . 18

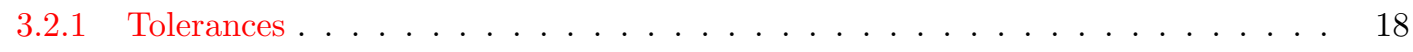

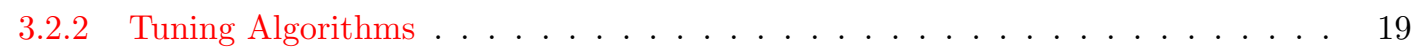

3.2.3 Beam Matrix Method ......................... 19

3.2.4 Analysis of the FFS using traditional methods . . . . . . . . . . . . . . 20

3.2.5 Tolerance Specification . . . . . . . . . . . . . . . . 21

3.2.6 Tuning example on NLC BDS . . . . . . . . . . . . . . . . . . . . 23

3.3 Beam Diagnosis . . . . . . . . . . . . . . . . . . . . . . . . 24

3.3.1 Twiss Parameters and Emittance at the Entrance of Final Focus(FF) Line . . 24

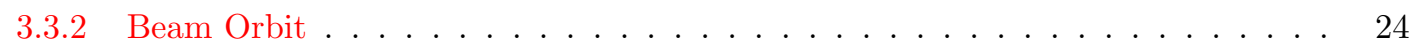


3.3 .3 Beam Size at IP . . . . . . . . . . . . . . . . . 26

3.3 .4 Cavity BPM at IP . . . . . . . . . . . . . . . . 26

3.3 .5 Other Monitors . . . . . . . . . . . . . . . . . 26

$\begin{array}{llr}4 & \text { Instrumentation } & 27\end{array}$

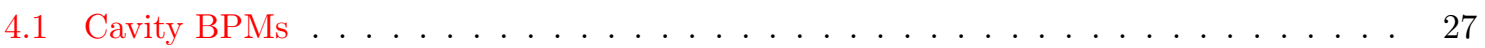

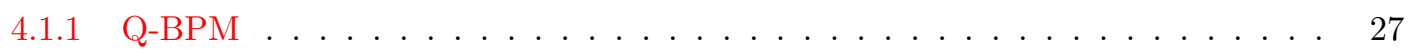

$4.1 .2 \quad \mathrm{IP}-\mathrm{BPM} \ldots \ldots \ldots \ldots \ldots \ldots \ldots \ldots$

4.2 Wakefield effects due to Cavity BPMs . . . . . . . . . . . . . . . . . . 30

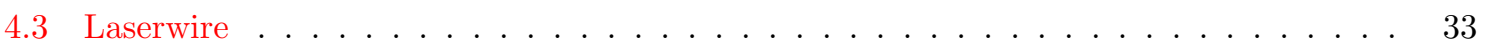

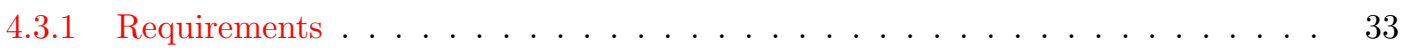

4.3.2 ATF Extraction line laserwire . . . . . . . . . . . . . . 35

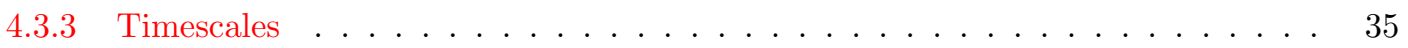

4.4 IP beam size monitor . . . . . . . . . . . . . . . . . . . . . . 36

4.4 Introduction $\ldots \ldots \ldots \ldots \ldots \ldots$

4.4 Compton scattering for ATF-2 beam conditions . . . . . . . . . . . . 36

4.4 .3 Laser system . . . . . . . . . . . . . . . . . . . . . 38

4.4 Laser system alternative . . . . . . . . . . . . . . . . . . 39

4.4.5 Launch Optics system . . . . . . . . . . . . . . . . . . . 39

4.4.6 Overlap with polarized source development . . . . . . . . . . . . . . 39

5 ATF extraction line \& extraction line diagnostics $\quad 41$

5.1 Emittance and orbit jitters in the extraction line . . . . . . . . . . . . . . . 41

5.1 .1 Vertical Emittance . . . . . . . . . . . . . . . . . 41

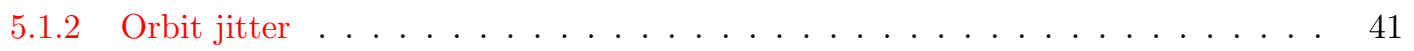

5.1 .3 Plan for improving the beam quality . . . . . . . . . . . . . . . 43

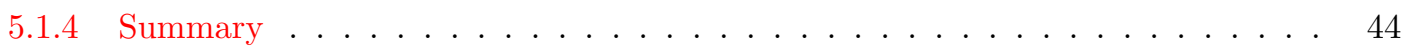

5.2 Vertical dispersion, 2nd order dispersion, and coupling correction in extraction line . . 44 
5.2.1 Measurement and correction of 2 nd order dispersion . . . . . . . . . . 46

5.2 .2 Design of an expanded diagnostics section . . . . . . . . . . . . . 47

5.2.3 Simulation of beam correction with new diagnostics . . . . . . . . . 50

$\begin{array}{lrr}6 & \text { Kicker } & 53\end{array}$

6.1 Kicker design, to produce the ILC-like train . . . . . . . . . . . . . . . . . . . 53

$\begin{array}{llr}7 & \text { Beam stabilization } & 57\end{array}$

7.1 Intra-train feedback and possible active stabilization . . . . . . . . . . . . . 57

7.1 .1 ATF2 jitter requirements . . . . . . . . . . . . . . . 57

7.1.2 Current ATF extraction line jitter situation . . . . . . . . . . . . 57

7.1.3 Intra-train beam feedback at ATF2 . . . . . . . . . . . . . . . 58

7.1.4 Ring-to-extraction-line feed-forward system . . . . . . . . . . . . 59

7.1.5 System integration issues ............................ 59

7.2 Alignment \& stabilization hardware and procedures . . . . . . . . . . . . . . . 60

7.2 .1 Initial alignment of magnets . . . . . . . . . . . . . . . . . 60

7.2.2 Control of position of quadrupole and sextupole magnets . . . . . . . . . 60

7.2.3 Control and Stabilization of the position of the final quadrupole magnets . . . 60

7.3 Ground motion in the ATF and ATF2 areas . . . . . . . . . . . . . . . . . 61

7.3 .1 Floor tilt measurements . . . . . . . . . . . . . . . . 61

7.3 .2 Vibration measurements .................... 63

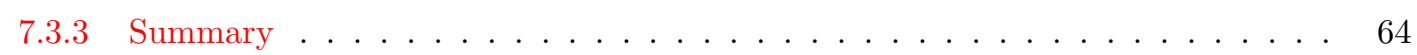

8 Strategy of Commissioning the ATF2 Beam $\quad 65$

$9 \quad r$ ATF2 magnets $\quad 69$

9.1 Introduction. . . . . . . . . . . . . . . . . . . . . . 69

9.1.1 Choice of magnets' effective length and apertures. . . . . . . . . . . . . 69

9.2 Performance Requirements of the ATF2 Magnets. . . . . . . . . . . . . . . . . . . 71 
9.3 Acquisition of the ATF2 magnets. . . . . . . . . . . . . . . . . . . . 71

9.4 Choice of Existing Quadrupole Design for most of the ATF2 Quads. . . . . . . . . . . 72

9.4.1 Details of the TOKIN 3390B style quad chosen for the new ATF2 quads. . . . 72

9.4.2 Field Quality of the TOKIN 3390B quadrupole design. . . . . . . . . . . . . 73

9.5 Meeting the Relative Field Errors Requirements and Power Supplies. . . . . . . . . . . 74

9.5.1 Meeting the Eddy Current and BBA Requirements. . . . . . . . . . . . . 77

9.6 Procurement of the ATF2 quadrupole magnets: potential vendor and schedule. . . . . 77

9.7 Choice of a design for the ATF2 chicane dipoles and FF bends. . . . . . . . . . . . . . 77

9.7.1 More information on the FFTB Magnet Power Supplies. . . . . . . . . . . . . . 78

9.8 Information on the SLAC FFTB Magnet Movers. . . . . . . . . . . . . . . . . . . . 78

10 ATF DR performance with ILC train $\quad 81$

10.1 Train format and emittance in ATF . . . . . . . . . . . . . . . . . . 81

10.2 Options for DR studies in ATF . . . . . . . . . . . . . . . . . 83

A Proposal of laser facility $\quad 85$

A.1 Description of the Project . . . . . . . . . . . . . . . . . . 85

A.2 High photon flux facilities . . . . . . . . . . . . . . . . . . . . . 85

A.3 Low photon flux facilities . . . . . . . . . . . . . . . . . . 88

A.4 Requirements .............................. . . . 88

B BINP kicker design proposal $\quad 89$

B.1 Low aperture extraction kicker . . . . . . . . . . . . . . . . . . 89

B.2 Wakes due to Extraction Kicker . . . . . . . . . . . . . . . . . . . . 93 


\section{List of Figures}

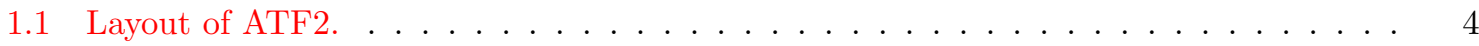

3.1 New final focus optics [5]. Chromaticity is corrected locally by the sextupoles in the final doublet region. . . . . . . . . . . . . . . . . . . 11

3.2 Classical CCX and CCY chromaticity correction optics. Cancellation of geometric aberrations by SF2 and SD1, etc. . . . . . . . . . . . . . .

3.3 ATF2 Optics showing the existing extraction line, extended diagnostic section and new final focus. . . . . . . . . . . . . . . . . . . . . . . . .

3.4 Bandwidth of the IP beta functions for the proposed ATF2 optics, as computed by SAD (left). Remaining second and third order aberrations corresponding to the $T_{i j k}$ and $U_{i j k l}$ matrix terms (right), computed by Transport code, where bars with different colors represent off-momentum particles with $\Delta E / E=0.2 \%$. . . . . . . . . . .

3.5 Bandwidth of proposed ATF2 optics computed with tracking by SAD (left) and TURTLE (right), with which it was optimized. . . . . . . . . . . . .

3.6 Field strength error (top plot) and magnet tilt error (bottom plot) giving $2 \%$ effect on beam size. Comparison of ATF2 optics and ILC optics. . . . . . . . . . . . . .

3.7 Jitter position error (top plot) static position error (bottom plot) giving $2 \%$ effect on beam size. Comparison of ATF2 optics and ILC optics. . . . . . . . . . . . . . .

3.8 Results from simulation of the effectiveness of R-matrix tuning knobs at restoring the nominal beam parameters under random error conditions (Horizontally: brown = error beam, red $=$ corrected beam, black =nominal beam. Vertically: green $=$ error beam, blue $=$ corrected beam, black $=$ nominal beam) $\ldots \ldots \ldots \ldots$. . . . . . . .

3.9 Tolerances on FF line quadrupoles in terms of individual quadrupoles (left) and as all quadrupoles together (right) and over all normal multipole orders. . . . . . . . . . . .

3.10 Tuning example for NLC BDS optics with errors shown in Table 3.8, showing horizontal and vertical beam sizes during the orbit correction and tuning procedure. The dash line shows the beam sizes without errors. See text for explanation of the knobs. The histogram bars show standard rms beam size for full beam and the x symbols show the gaussian fit sigma for the beam core. . . . . . . . . . . . . . . .

3.11 An example of WS signals. From top to bottom, signals from $y,+10 \mathrm{deg},-10 \mathrm{deg}$, and $x$ wire are shown. . . . . . . . . . . . . . . . . .

3.12 An example of WS analysis. Five WSs are used in this analysis. . . . . . . . . . .

4.1 Cavity BPM attached on a quadrupole magnet. . . . . . . . . . . . . . 
4.2 Structure of the Q-BPM. . . . . . . . . . . . . . . . . . . . 28

4.3 Electronics of the Q-BPM. . . . . . . . . . . . . . . . . . . . . 29

4.4 Electric field of the dipole mode in the IP-BPM. . . . . . . . . . . . . . . . . . . 30

4.5 Layout of the IP-BPM. . . . . . . . . . . . . . . . . . . . . . . . . . . . . . 31

4.6 Electronics for IP-BPM. . . . . . . . . . . . . . . . . . . . . . . . 31

4.7 Geometry used for wakefield calculation. . . . . . . . . . . . . . . . . . . . 32

4.8 Transverse wake of one cavity BPM module. The bunch shape, with head to the left, is given by dashes. . . . . . . . . . . . . . . . . . . . . . . . 32

4.9 Proposed location of laserwire $(\mathrm{s}) \ldots \ldots \ldots \ldots$

4.10 Ratio of Compton and Thompson cross section as a function of beam energy. . . . . . 37

4.11 Compton cross section as a function of laser wavelength. . . . . . . . . . . . . . 37

4.12 Conceptual diagram of laser system components. . . . . . . . . . . . . . . . . . . . 38

4.13 Scheme of launch system optical layout showing crossing angles of $6^{\circ}, 30^{\circ}$ and $174^{\circ} \cdot \quad 39$

5.1 Vertical emittance vs. bunch intensity $N$, measured in the extraction line using wire scanners (EXT) and measured in the damping ring using the laserwire monitor (DR-LW). 42

5.2 Layout of existing EXT line showing locations of various R\&D experiments. . . . . . 44

5.3 Existing ATF EXT diagnostic section showing skew quads (SQ), wire scanners (WS), and betatron phases. . . . . . . . . . . . . . . . . 45

5.4 Results of a typical measurement of 1st and 2nd order horizontal dispersion in EXT. . 46

5.5 Variation of horizontal beam position with energy offset at 2 diagnostic section BPMs showing quadratic dependence. This illustrates the procedure for measuring 2 nd order dispersion and typical measurement errors. . . . . . . . . . . . . . . . . . . 46

5.6 Measured second order dispersion and its derivative (along z) versus strength of sex-

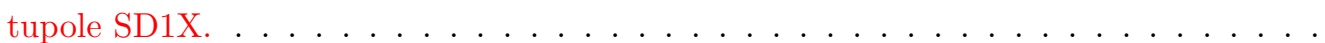

5.7 Ideal skew correction / emittance measurement section for NLC (top plot) and the new ATF2 skew correction / emittance measurement section (bottom plot). . . . . . . .

5.8 ATF2 extraction line (EXT2) with expanded skew correction / emittance measurement section showing location of an additional quad. The partial -I transfer matrix between kickers for this optics is also shown. . . . . . . . . . . . . . . . .

5.9 Simulation of correction in EXT2, beam orbit before and after steer/launch correction. 51 
5.10 Results of simulated corrections in EXT2, vertical IP spot size versus seed number at various stages of the procedure . . . . . . . . . . . . . . . . 52

6.1 Results of tests of MOS-FET power transistor-based pulser at ATF. . . . . . . . . . 54

7.1 Measurement locations for tilt meters and accelerometers. . . . . . . . . . . . . . . 62

7.2 Floor tilt measurements in the ATF area (a) and ATF2 area (b). Blue and red lines show floor tilt and outside air temperature, respectively. . . . . . . . . . . .

7.3 Integrated amplitude measured in the ATF beam line, in the ATF2 area and in the clean room. The ground motion is smallest in the ATF beam line, where the floor is reinforced. . . . . . . . . . . . . . . . . . . .

7.4 Amplitude ratio of the girder motion relative to that of the floor (ATF beam line). . . 64

9.1 Snapshot of top assembly drawing of the TOKIN 3390B quad. . . . . . . . . . . 73

9.2 Snapshot of coil drawing of the TOKIN 3390B quad. . . . . . . . . . . . . . . . . 74

9.3 PHOTOS of a TOKIN 3390B quadrupole in use at ATF, KEK. . . . . . . . . . . . . 75

9.4 FFTB magnet mover. . . . . . . . . . . . . . . . . . . . . . . . . 78

A.1 Schematic Layout of the laser interaction region and recirculating cavities. . . . . . 86

A.2 Expected photon energy spectrum simulated by CAIN. . . . . . . . . . . . . . 87

B.1 Cross-section of the vacuum chamber with built-in low aperture kicker (left) and close view of the kicker part with dimensions (right) . . . . . . . . . . . . 89

B.2 Longitudinal cross-section of the vacuum chamber with built-in low aperture kicker and schematics of the beam orbits for the nominal and extracted beam. . . . . . . . .

B.3 Two groups of kickers, working on odd and even pulses, allow halving the repetition rate of the switches. The difference of the drift length can be corrected downstream. .

B.4 Calculated field in the low aperture kicker. . . . . . . . . . . . . . . . . . . . . 91

B.5 Kicker pulse shape with fixed amplitude of the traveling wave pulse and for various length of the kicker $(15,20,25,30,40,60 \mathrm{~cm})$. Calculated for quasi-square pulse with $2.5 \mathrm{~ns}$ FWHM duration and with raise/fall (with $\sin ^{2}$ shape) duration of $1.5 \mathrm{~ns}$. This picture shows that the length of the kicker should not be longer than $20 \mathrm{~cm}$. . . . . .

B.6 Geometry used for wakefield calculation. . . . . . . . . . . . . . . . . . . 94 


\section{List of Tables}

2.1 Beam parameters achieved at ATF and planned for ATF2, goals $\mathbf{A}$ and $\mathbf{B}$. The ring energy is $E_{0}=1.3 \mathrm{GeV}$, the typical bunch length and energy spread are $\sigma_{z} \sim 8 \mathrm{~mm}$ and $\Delta E / E=0.08 \% \ldots \ldots \ldots \ldots \ldots \ldots \ldots \ldots$

3.1 ATF2 proposed IP parameters compared with ILC. . . . . . . . . . . . . . . . . . . . 12

3.2 Jitter tolerance specification. . . . . . . . . . . . . . . . . . . . . . 21

3.3 Fast error tolerance specification. . . . . . . . . . . . . . . . . . . . . . . . 21

3.4 Fast error tolerance specification. . . . . . . . . . . . . . . . . . . . . 21

3.5 Tunable errors tolerance specification. . . . . . . . . . . . . . . . . . . . . 22

3.6 Tolerance specifications for the quadrupole magnets. . . . . . . . . . . . . . . . . . 23

3.7 Tolerance specifications for the Sextupole magnets. . . . . . . . . . . . . . . . . . 23

3.8 Errors used in tuning simulations of NLC BDS optics. . . . . . . . . . . . . . . . . . . 24

4.1 Laser spot-sizes for green laser light of wavelength $532 \mathrm{~nm}$ and optimised laser optics, assuming an electron-bunch aspect ratio $\sigma_{x}^{e} / \sigma_{y}^{e}$ of $10 \ldots \ldots \ldots \ldots$

4.2 ATF-2 conditions (compared to FFTB conditions) . . . . . . . . . . . . . . 37

4.3 Minimum measurable spotsize using $532 \mathrm{~nm}$ photons for modulation depths $10 \%$ and $90 \% \ldots \ldots \ldots \ldots \ldots \ldots \ldots \ldots$

6.1 Kicker parameter comparison. . . . . . . . . . . . . . . . . . . 53

6.2 Ring and extracted bunch timing. . . . . . . . . . . . . . . . . . . 55

9.1 List of new magnets in the optimal beamline. . . . . . . . . . . . . . . . . . 70

9.2 Achievable magnet stability if (unmodified) FFTB power supplies are used. Here $I_{O}$ is operating current with suggested ATF magnet design. $\triangle B / B_{F F T B}-$ PS stability at the operating current if a $250 \mathrm{amp}$ FFTB PS is used. $\Delta B / B$ - Tolerable relative field error. Magnets showing in italic do not meet their published stability tolerance if powered by FFTB power supplies. . . . . . . . . . . . . . . . . . . 76

10.1 Parameters of the injected beam. . . . . . . . . . . . . . . . . . . 81

10.2 Parameters and achieved performance of ATF-DR . . . . . . . . . . . 83

A.1 Parameters for high intensity photon facility. . . . . . . . . . . . . . . . . . 86

B.1 Tentative parameters of the low aperture kicker for the ATF2. *The number of modules depends on the possibility to provide orbit correction and modify the septum. . . . . . 


\section{Introduction \& Executive Summary}

\section{$1.1 \quad$ Preamble}

This document is the first of two volumes describing the ATF2 project. The present volume discusses the technical justification for ATF2 and presents a design description.

Since the International Committee for Future Accelerator (ICFA) decision on the choice of technology, a world-wide collaboration on the design of the International Linear Collider (ILC) has rapidly progressed [1]. The formation of the Global Design Effort (GDE) will accelerate the work towards a final design. An important technical challenge is obviously the high gradient acceleration but what is similarly challenging is the collision of extremely small beams of a few nanometer size. The latter challenge has three distinct issues: creating small emittance beams, preserving the emittance during acceleration and transport, and focusing the beams to nanometers. Most studies have been done using computer simulations but many issues still remain that require experimental verification. Accelerator Test Facility (ATF) at KEK was built to create small emittance beams, and succeeded in obtaining an emittance that almost satisfies the ILC requirements [2]. In this proposal we present a project, ATF2, which addresses the focusing of the beam into a nanometer spot.

The ATF2 project will extend the extraction beamline of the ATF with an ILC-type final focus system to create a tightly focused, stable beam by making use of the small emittance of the ATF. The layout is shown in Figure 1.1. ${ }^{1}$ In the longer term, the ATF2 project would also provide invaluable input for the CLIC design of a future multi-TeV collider.

\subsection{Previous Facilities}

The ATF2 facility will be a continuation of the successful results achieved at the Final Focus Test Beam (FFTB) at SLAC [3]. The FFTB, which achieved a beam size of $70 \mathrm{~nm}$, provided invaluable experience and confidence in design and operation of the final focus. However, it could not address questions of reliably maintaining the beam size over the long term or of beam stability. Between 1994 and 1997, there were a number of short runs of 1-3 weeks duration. The small beam size was achieved in about half of the runs. The measured beam size was also much larger than the $40 \mathrm{~nm}$ value expected given the input beam emittance. The difference was attributed to significant jitter of the focused beam and was also partly due to limited accuracy in tuning the linear optics and

\footnotetext{
${ }^{1}$ Another layout which is slightly to the south (lower in the figure) has also been discussed. It was designed to avoid a possible conflict with existing facilities should ATF2 be built before the other work is completed. In this proposal we present results for the layout shown in the figure, anticipating that issues such as tolerances will be essentially the same for either layout.
} 


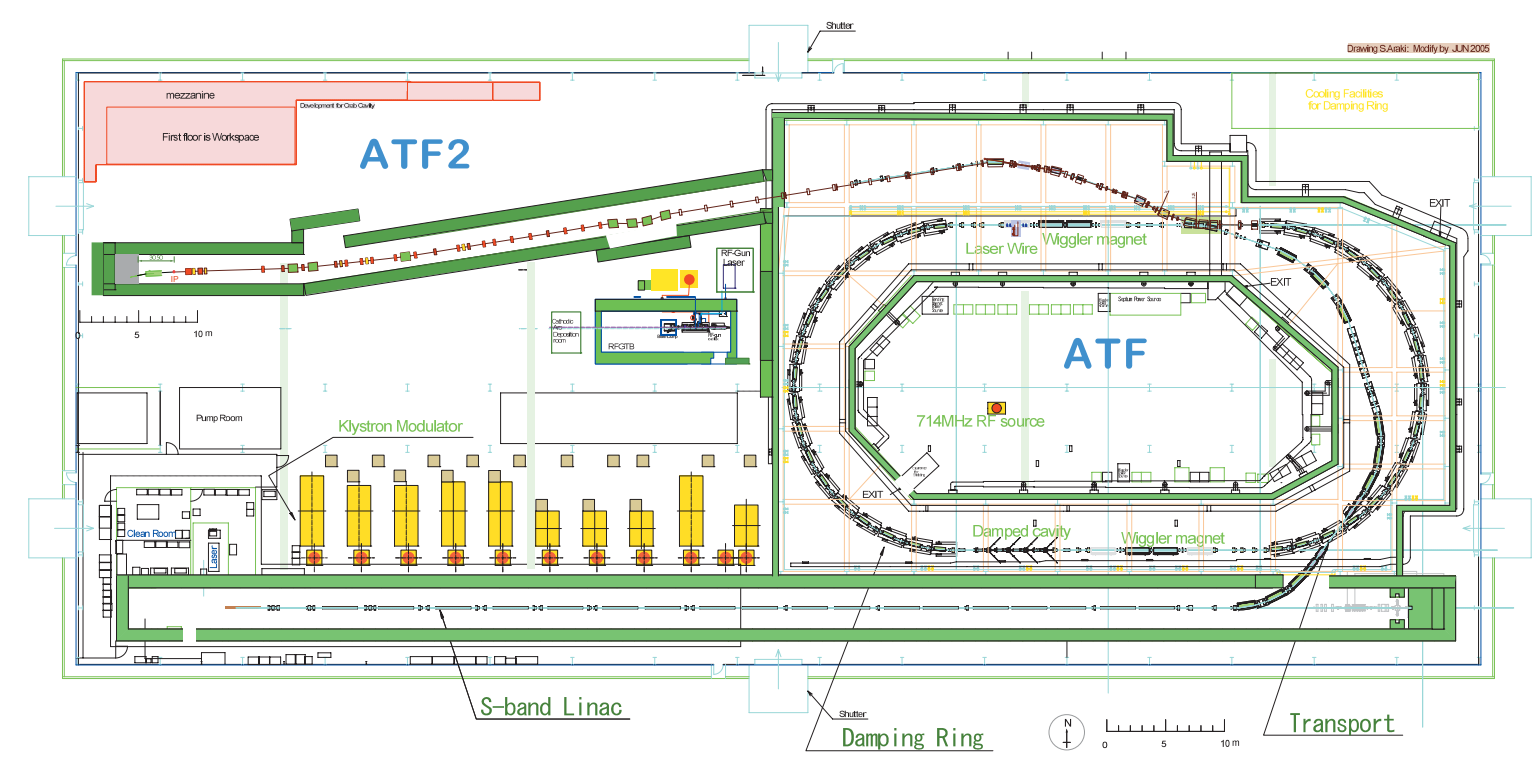

Figure 1.1: Layout of ATF2.

the aberrations [4]. Since FFTB runs were not compatible with the Stanford Linear Collider (SLC) operation, detailed investigations of these important issues could not be pursued.

Since the FFTB era, the ILC Beam Delivery System (BDS) design has changed significantly. The recently proposed compact final focus optics with local chromaticity correction [5] has better performance in a much shorter system and can more easily be extended to multi-TeV. This is now the basis of the ILC BDS design but has never been tested experimentally. Prior to ILC construction, it would be important to obtain real experience with the compact final focus optics. The ATF2 facility would give important operating experience during preparation of the ILC Technical Design Report (TDR), construction and early years of operation, and will become the alma mater for the generation of physicists needed to complete the ILC design and operate the ILC.

\subsection{Goals of ATF2}

The ATF2 will address two major challenges of the ILC BDS: focusing the beams to nanometer size and providing sub-nanometer stability. The ATF has the high quality beams required to achieve these goals - it has successfully produced beams with the lowest emittance ever achieved, in both single and multi-bunch $[2,6]$ modes, which are very similar to those for ILC. ATF2 will require further development of hardware and diagnostics and improvement of the extracted beam quality. The schedule for ATF2 and hardware development will successively address the two major goals:

(A) Achievement of $37 \mathrm{~nm}$ beam size

(A1) Demonstration of a compact final focus system based on local chromaticity correction 
(A2) Maintenance of the small beam size

(B) Control of beam position

(B1) Demonstration of beam orbit stabilization with nano-meter precision at the IP

(B2) Establishment of beam jitter controlling techniques at the nano-meter level with an ILC-like beam

Ultimately, the ATF2 will aim at achieving the small beam size and nanometer beam stability simultaneously [7].

\subsection{Requirements on the ATF Beam}

Achieving these goals will place additional requirements on the diagnostic hardware and the ATF extraction beam quality.

First, the target beam size assumes a value of $3 \times 10^{-8} \mathrm{~m} \cdot \mathrm{rad}$ for the vertical normalized emittance. Although the present emittance of the stored beam has been measured to be $1.5 \times 10^{-8} \mathrm{~m} \cdot \mathrm{rad}$, the extracted beam is considerably larger (factor $\sim 3$ more than in the ring). While an effort will be made to improve the extracted emittance, the ATF2 proposal assumes only a factor of 2 reduction in the extracted beam emittance compared to present performance.

More important is the bunch-to-bunch position jitter of the extracted beam, quoted for the target beam emittance. For the goal A the r.m.s fluctuation has to be less than $\sim 1 / 3$ sigma, which is nearly satisfied by the present beam. The goal $\mathbf{B}$, on the other hand, requires jitter less than $\sim 1 / 20$ sigma, which imposes tight constraints on the extraction kicker, etc. This requirement will take time to achieve. One may have to wait for B2 where a feedback system is implemented on an ILC-like beam to fully achieve the B goals. A Beam Position Monitor (BPM) with resolution of a few nanometers is also required at the Interaction Point (IP). These requirements are discussed below in the main body of the Proposal.

\subsection{Comparison with ILC FFS}

Many features of the ATF2 are common to the ILC Final Focus System (FFS) in spite of the two orders of magnitude lower beam energy. As stated above, the principle of the ATF2 optics design is identical to that for the ILC. The natural chromaticity and the relative beam energy spread are quite similar. Most of the tolerances of the subsystems are comparable, such as the tolerances on magnetic field, jitter vibration and power supply stability. Since the absolute beam size at the IP is larger by factor of 5, the tolerance on magnet position jitter for goal $\mathbf{A}$ is obviously somewhat looser. However, the jitter tolerance for goal $\mathbf{B}$ is similar to ILC. The required resolution for the BPMs attached to the quadrupole magnets are about the same as for ILC. Thus, the success of ATF2 is directly related to ILC. 
On the other hand, some conditions at ATF2 will be different from the situation at the ILC FFS. Since the ATF2 beam does not come from a long linac, it will not show the time variation due to the integrated effects of ground motion and wakefields over the long ILC linac. Since the total length of the ATF2 final focus system is an order of magnitude shorter than for ILC, the differential ground (floor) motion can be considerably smaller.

The ATF2 is also susceptible to complications which are absent in the ILC. For example, ATF2 requires a BPM at the IP with a resolution of a few nanometers. In the real machine, this BPM is not needed, because the beam-beam interaction will act as a position diagnostic. To achieve the goal (B1) it is necessary to suppress the bunch-to-bunch position fluctuations at the exit of the damping ring below $\sim 1 / 20$ of the r.m.s. beam size, which is tighter than in the ILC by a factor of 2-3. This is because the geometric emittance of the ATF2 beam is much bigger than that at the ILC interaction point. Also, the ATF bunch length $\left(\sigma_{z}\right)$ is $\sim 8 \mathrm{~mm}$ which is much longer than that of the ILC FF: i.e. $\sigma_{z}=150$ to $500 \mu \mathrm{m}$. The longer bunch length demands a design of a cavity-type BPM with a lower resonant frequency. Environmental changes at the ATF building, such as temperature fluctuations and ground motion, are expected to be more severe than in the ILC tunnel, which will be underground. All these effects make the ATF2 more difficult than the ILC FFS.

In spite of all the differences stated above, the ATF2 will be a very good model for the ILC FFS. We believe the success of ATF2 will greatly help the design and operation of the ILC FFS.

\subsection{Scope, Timeline and Budget}

The timeline of the construction will be described in the second volume. Here, we briefly mention the relationship to the proposed timeline of the GDE.

The scientific goals of the ATF2 have little to do with the BCD (Baseline Conceptual Design) which is to be completed by the end of 2006. For example, ATF2 cannot exert a large influence on the choice of the crossing angle. On the other hand, ATF2 can have a big impact on the TDR (Technical Design Report) which is to be finished in 2008 or later. Thus, the goals A and B for ATF2 should be accomplished well before the TDR. However, the mission of ATF2 will not finish then. The effort of improving the final focus design will still continue until the beamline components are to be ordered from industry, which will be considerably later than the TDR. Moreover, the experience with tuning procedures for ATF2 will serve to minimize the commissioning period of the ILC FFS. Thus, the study at ATF2 will continue even after the start of ILC construction.

In addition, the high quality beams available at ATF2 would generate many other opportunities for experiments. As a future option, a photon linear collider (PLC) test facility is also being considered. At the PLC test facility, a photon beam would be produced from the Compton scattering with a laser beam synchronized to the electron beam, with high intensity and multi-bunch structure similar to the ILC. Experiments could also be conducted to test QED in the strong field of a high intensity laser.

The cost and the organizational issues will also be described in detail in the second volume. The total cost of the ATF 2 construction is estimated to be about $4 \times 10^{8}$ Yen (about $4 \mathrm{M}$ US\$). This number 
includes all the hardware components and the infrastructure such as the floor refurbishment, but it does not include the staff salaries or contingency. KEK would cover the expenses of the infrastructure but the rest of the costs would be shared more or less equally among the three regions, Asia, Americas and Europe.

\subsection{Summary}

This document describes the proposal for an international final focus ATF2 facility for consideration by the worldwide International Linear Collider collaboration. The ATF2 facility will benefit from the uniquely small beam emittances achievable at KEK ATF, and will provide valuable experience in achieving, maintaining and stabilizing nanometer scale beams. Such a facility will be invaluable for the successful design and operation of ILC, provide a test bed for development of instrumentation and accelerator physics ideas, train the next generation of accelerator physicists and promote truly international collaboration building a new facility. 


\section{Overview of the ATF2 project}

The ATF2 design and schedule for ATF2 hardware development address two major goals described in detail in Section 1, namely achieving a $37 \mathrm{~nm}$ beam size (goal A) and nanometer control of beam position (goal $\mathbf{B}$ ). The ATF has the high quality beams required to achieve these goals - it has successfully produced beams with the lowest emittance ever achieved, in both single and multi-bunch $[2,6]$ modes, which are very similar to those for ILC. Further hardware and diagnostics must be developed and the extracted beam quality improved for ATF2. The ATF and ATF2 beam parameters are shown in Table 2.1.

The ATF2 final focus design is based on the recently proposed compact final focus optics with local chromaticity correction [5], which now serves as the basis for the ILC FF design. The optics design is described in Section 3.

In addition to the final focus proper, the ATF2 design includes an extended new diagnostics section, that would allow coupling correction of the beam and accurate measurements of its properties. Design of this diagnostics section is described in Section 5.2.

To fully realize the ATF2 program and fulfill both goals $\mathbf{A}$ and $\mathbf{B}$, a number of hardware developments and other improvements are needed to the ATF damping ring and extraction system.

For goal $\mathbf{A}$, an interferometer-based beam size monitor (BSM, also called Shintake monitor, described in Section 4.4) will be installed at the IP. The laser will operate with higher modes than those used for the FFTB measurements [8]. To measure the beam orbit and maintain the beam size with feedback, the beamline magnets will be equipped with $100 \mathrm{~nm}$ resolution cavity-BPMs (see Section 4.1.1) and will be placed on movers. Tuning methods will be established based on BSMs as well as BPMs, as discussed in Section 3.2. To achieve the goal B1, a set of precision "nano-BPMs" will be installed at the IP. A set of BPMs with a resolution of better than $2 \mathrm{~nm}$ are now being developed by the Japan-US-UK group (see Section 4.1.2). The possibility of combining the two goals, with both the BSM at the IP and nano-BPMs nearby to achieve both the small beam size and nanometer stability, is under investigation.

The beam quality must also be improved for ATF2. The present normalized emittance in the ATF extraction line is estimated to be $4.8 \times 10^{-8} \mathrm{~m}$, three times larger than that in the damping ring. The emittance can be reduced to the nominal value of $3 \times 10^{-8} \mathrm{~m}$ by correcting the $\mathrm{x}-\mathrm{y}$ coupling. The beam jitter must be reduced to about $30 \%$ of the beam size for goal $\mathbf{A}$, and $5 \%$ for goal $\mathbf{B}$. The vertical beam jitter in the ATF extraction line is now typically about $30 \%$ of the beam size (up to $100 \%$ on a time scale of several minutes), which is much larger than the $10 \%$ beam jitter observed in the damping ring. The slow drift is believed to come from the extraction kicker system, which may be improved by a double kicker scheme together with an additional feed-forward system. This is expected to reduce the jitter to the same level as in the damping ring. This is discussed further in Section 7.1. 
Table 2.1: Beam parameters achieved at ATF and planned for ATF2, goals A and $\mathbf{B}$. The ring energy is $E_{0}=1.3 \mathrm{GeV}$, the typical bunch length and energy spread are $\sigma_{z} \sim 8 \mathrm{~mm}$ and $\Delta E / E=0.08 \%$.

\begin{tabular}{llll}
\hline & Measured & $(\mathbf{A})$ & $(\mathbf{B})$ \\
\hline Single Bunch & & & \\
$N_{\text {bunch }}\left[10^{10}\right]$ & $0.2-1.0$ & 0.5 & 0.5 \\
DR $\gamma \varepsilon_{y}\left[10^{-8} \mathrm{~m}\right]$ & 1.5 & 3 & 3 \\
Extr. $\gamma \varepsilon_{y}\left[10^{-8} \mathrm{~m}\right]$ & $3.0-6.5$ & 3 & 3 \\
Multi Bunch & & & \\
$n_{\text {bunches }}$ & 20 & $1-20$ & $3-20$ \\
$N_{\text {bunch }}\left[10^{10}\right]$ & $0.3-0.5$ & 0.5 & 0.5 \\
DR $\gamma \varepsilon_{y}\left[10^{-8} \mathrm{~m}\right]$ & $3.0-4.5$ & 3 & 3 \\
Extr. $\gamma \varepsilon_{y}\left[10^{-8} \mathrm{~m}\right]$ & $\sim 6$ & 3 & 3 \\
\hline IP $\sigma_{y}^{*}[\mathrm{~nm}]$ & & 37 & 37 \\
IP $\Delta y / \sigma_{y}^{*}[\%]$ & & 30 & 5 \\
\hline
\end{tabular}

The beam stability can be further improved with a new 300 ns kicker which will make it possible to extract the beam-train in 3 bunches separated by 150 ns. A fast feedback system being developed by KEK and UK groups, FEATHER and FONT, will be used to further stabilize the third bunch. The nano-BPM system will be able to verify the performance of the fast feedback system at the nanometer level. Further beam jitter control at the nanometer level with an ILC-type beam (goal B2) will require a very fast kicker with less than a ns rise time and stable pulse height. This will make it possible to extract an ILC-type train, i.e. 20 bunches with about $300 \mathrm{~ns}$ separation at $5 \mathrm{~Hz}$, see Section 6.1. These developments may evolve over several years including continuing during ILC construction.

Finally, before installation of the ATF2 components, the floor under the new beamlines must be reinforced similarly to what was done for the ATF damping ring. Studies with seismometers and tiltmeters, described in Section 7.3 have shown that the present floor in the ATF2 area is much less stable and has a large sensitivity to temperature variation. Reinforcement of the floor will help mitigate stability issues.

The high quality beams available at ATF2 would generate many other opportunities for experiments. As an option, a photon linear collider (PLC) test facility has been considered, and described in Section A. At the PLC test facility, a photon beam would be produced with high intensity and multibunch structure similar to the ILC. Experiments could also be conducted to test QED in the strong field of a high intensity laser. 


\section{Optics}

\subsection{ATF2 FF optics and comparison with the ILC-FF}

\subsubsection{ATF2 Optics Design}

The ATF2 will be the test bench for the ILC final focus (FF) design. The optics design is the Next Linear Collider (NLC) compact final focus [5] scaled down to match the beam energy of $1.3 \mathrm{GeV}$ and fit within a beamline length of about $36 \mathrm{~m}$ available at the KEK ATF. The optics is based on the novel concept of chromatic correction sextupoles interleaved with the final doublet (FD) quadrupoles, leading to a more compact final focus design, instead of the classical scheme with separate chromatic correction sections for the horizontal and vertical planes CCX and CCY (see Figs. 3.1 and 3.2).

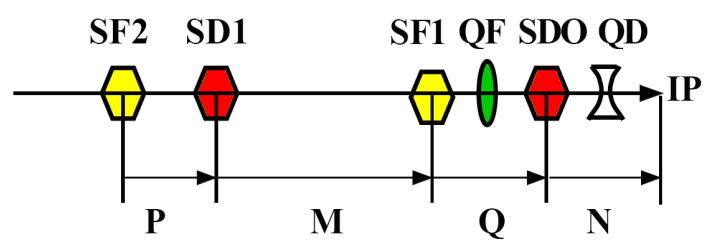

Figure 3.1: New final focus optics [5]. Chromaticity is corrected locally by the sextupoles in the final doublet region.

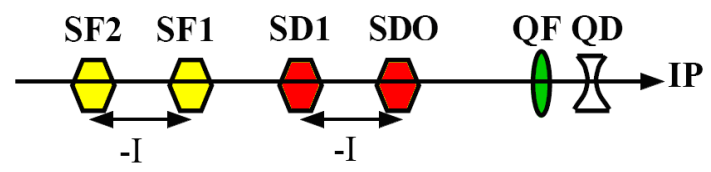

Figure 3.2: Classical CCX and CCY chromaticity correction optics. Cancellation of geometric aberrations by $\mathrm{SF} 2$ and $\mathrm{SD} 1$, etc.

One of the critical issues in designing a final focus optics is how to suppress the beam size growth due to the beam energy spread $\delta_{E}=\left(E-E_{0}\right) / E_{0}$. The beam growth is approximately expressed as

$$
\frac{\Delta \sigma_{x, y}^{*}}{\sigma_{x, y}^{*}} \approx W_{x, y} \delta_{E}
$$

where $W$ is the chromaticity and $\sigma^{*}$ is the geometrical beam size at the IP. The value of the chromaticity is approximately $W \sim L^{*} / \beta^{*}$ and for a typical final focus line the vertical chromaticity is in the order of $10^{4}$. Thus even with a small energy spread $\delta_{E}=10^{-3}$ the beam size may easily grow by an order of magnitude. The chromaticity is corrected by introducing sextupole magnets in dispersive regions. Most of the chromaticity comes from the final doublet quadrupole magnets, therefore it is 
most effective to have the sextupoles in the final doublet, providing local compensation of chromaticity. The second order dispersion, arising from the sextupoles, can be compensated simultaneously with the chromaticity if one allows half of the total horizontal chromaticity to come from upstream of the FD. Higher order aberrations can be cancelled by respecting proper beam transport relationships between the downsteam and upstream sextupoles. Advantages of the local chromaticity compensation are that the optics is less sensitive to synchrotron radiation, can be reasonably short even for $\mathrm{TeV}$ energy, and can have a large bandwidth (of about a percent or higher).

\subsubsection{Proposed optics designs}

The final focus beam line of the ATF2 will extend the existing ATF extraction line as shown in Fig. 1.1. The optics of the ATF2 final focus with the new diagnostics section is shown in Fig. 3.3. The $\mathrm{FF}$ optics has $L^{*}=1 \mathrm{~m}$ (distance from last focusing quadrupole to the IP), $\eta^{\prime}=-0.14$ (derivative of dispersion at IP) with IP beta-functions $\beta_{x / y}^{*}=4 / 0.1 \mathrm{~mm}$. The total chromaticity of this optics is approximately the same as in the ILC FF. The vertical beam size will be focused to $37 \mathrm{~nm}$ with an aspect ratio of about 100:1 similar to the ILC. The ATF2 beam parameters are compared with ILC parameters in Table 3.1.

The ATF2 proposal originally considered an alternative final focus optics proposed by Kuroda et al. in [9]. We have compared the performance of the two designs and found that the optics suggested in [9] has fewer magnets and would be less expensive. However in this optics, the chromaticity correction is not purely local, the tolerances on magnet strength and position are tighter, the bandwidth is narrower and scaling to TeV energy is more difficult. Therefore, the NLC-like optics was chosen as a baseline design for the ATF2. A detailed report comparing these two optics designs is in preparation [10].

Table 3.1: ATF2 proposed IP parameters compared with ILC.

\begin{tabular}{lll}
\hline Parameters & ATF2 & ILC \\
\hline Beam Energy $[\mathrm{GeV}]$ & 1.3 & 250 \\
$\mathrm{~L}^{*}[\mathrm{~m}]$ & 1 & $3.5-4.2$ \\
$\gamma \epsilon_{x}[\mathrm{~m}-\mathrm{rad}]$ & $3 \times 10^{-6}$ & $1 \times 10^{-5}$ \\
$\gamma \epsilon_{y}[\mathrm{~m}-\mathrm{rad}]$ & $3 \times 10^{-8}$ & $4 \times 10^{-8}$ \\
$\beta_{x}^{*}[\mathrm{~mm}]$ & 4.0 & 21 \\
$\beta_{y}^{*}[\mathrm{~mm}]$ & 0.1 & 0.4 \\
$\eta^{\prime}(\mathrm{DDX})[\mathrm{rad}]$ & 0.14 & 0.094 \\
$\sigma_{E}[\%]$ & $\sim 0.1$ & $\sim 0.1$ \\
Chromaticity $W_{y}$ & $\sim 10^{4}$ & $\sim 10^{4}$ \\
\hline
\end{tabular}

The ATF2 optics was designed primarily using codes MAD, Transport, Turtle and DIMAD. However, we have also used different accelerator codes to verify the optics and perform beam tracking of the ATF2 beam line, for example the ELEGANT code and in particular the SAD code, which is widely 


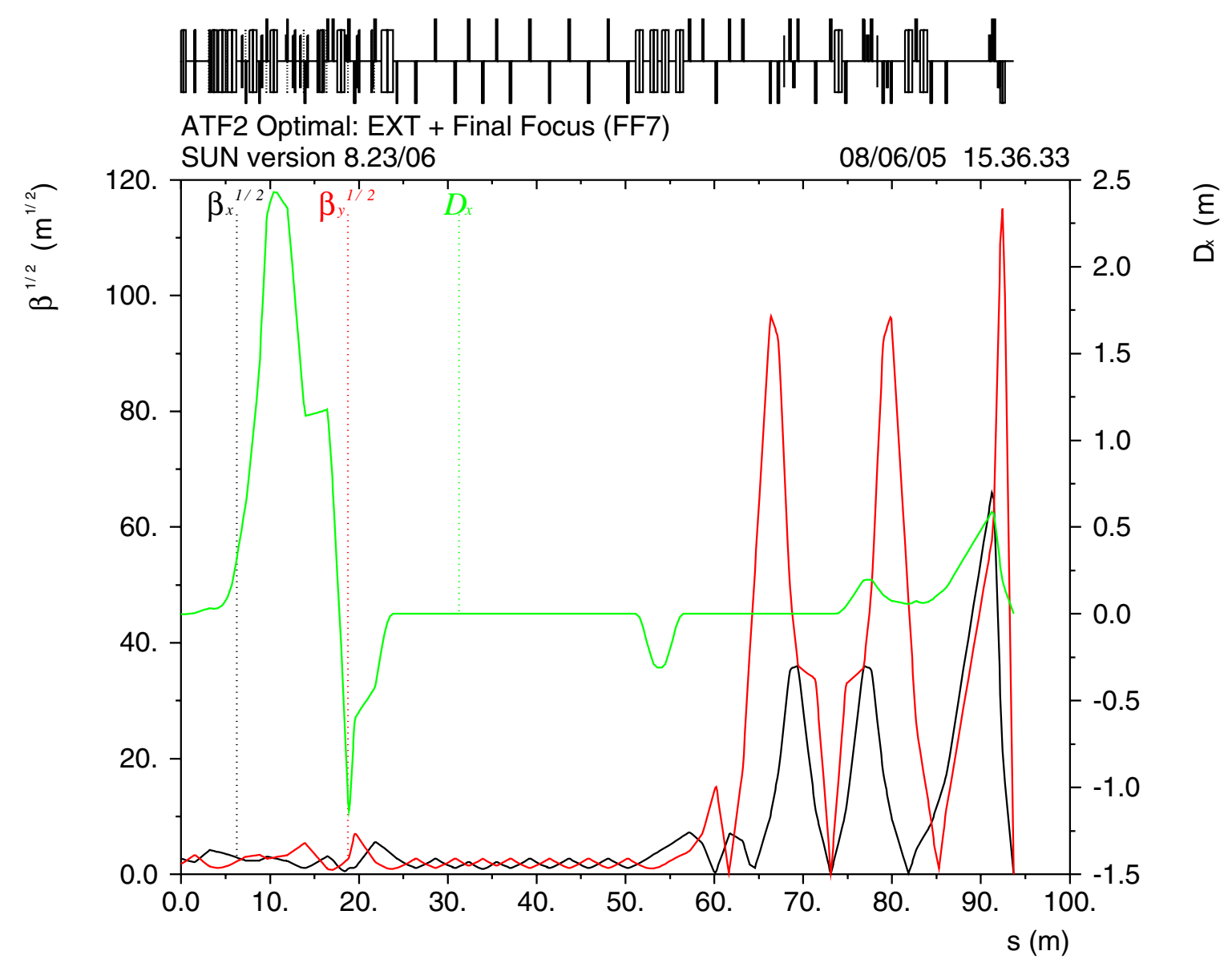

Figure 3.3: ATF2 Optics showing the existing extraction line, extended diagnostic section and new final focus. 
used at KEK [11]. Such cross-checks also had the benefit of facilitating and easing the communications and exchange of information between the optics designers. From this cross-comparison we have found that there is a noticeable but not major difference between the codes for large momentum offset. The tracking simulations are given in the next section and details of the code comparison will be presented in [10].

\subsubsection{Bandwidth and tracking results}

The complete optics of the extraction line and final focus has been optimized to achieve large momentum acceptance ("bandwidth"). To maximize the overall bandwidth, the chromatic properties of the existing extraction line (in particular the second order dispersion) needed to be corrected. To suppress the 2nd order dispersion and minimize the vertical chromaticity at the exit of extraction line, three additional sextupoles have been inserted in the extraction line, as described in Section 5.2. The optics and sextupoles in the final focus then have been optimized to cancel the overall chromaticity, second order dispersion and higher order aberrations.

The bandwidth, in terms of the horizontal and vertical beta functions versus energy offset, is shown in Fig. 3.4 (left) for the total system. This bandwidth is computed with SAD, and it should be noted that the bandwidth calculated with MAD and Turtle, which were used to optimize the design, is usually somewhat wider. The remaining second and third order aberration terms, in arbitrary relative units, are shown in Fig. 3.4 (right).

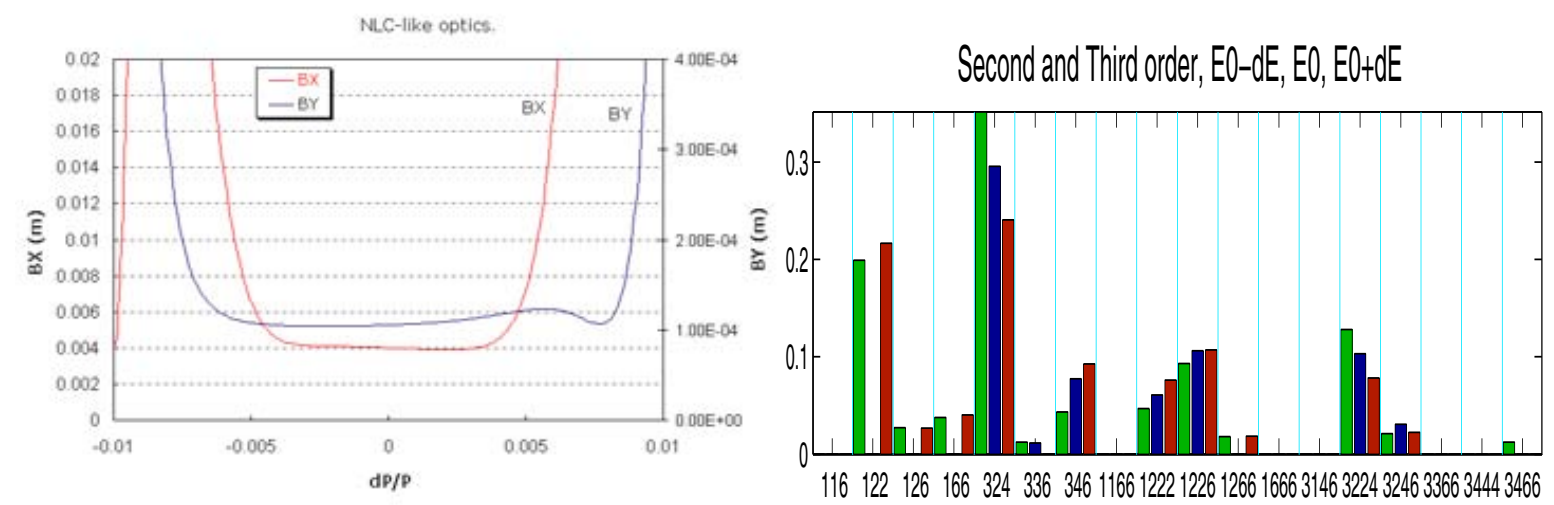

Figure 3.4: Bandwidth of the IP beta functions for the proposed ATF2 optics, as computed by SAD (left). Remaining second and third order aberrations corresponding to the $T_{i j k}$ and $U_{i j k l}$ matrix terms (right), computed by Transport code, where bars with different colors represent off-momentum particles with $\Delta E / E=0.2 \%$.

Beam tracking has been performed from the existing ATF extraction line through the final focus system to the IP with normalized emittancies $\gamma \varepsilon_{x}=3 \times 10^{-6} \mathrm{~m}$ and $\gamma \varepsilon_{y}=3 \times 10^{-8} \mathrm{~m}$ as achieved in the ATF ring. To be able to run with either accelerator code, the optics was converted from MAD to $\mathrm{SAD}$ and vice-versa. If it is not mentioned otherwise, we will refer to the luminosity equivalent beam 
size as used in DIMAD [12] and defined as

$$
\sigma_{\text {les }}=\frac{1}{2 \sqrt{\pi} \int_{\infty}^{-\infty} \rho^{2}(y) d y}, \quad \int_{\infty}^{-\infty} \rho^{2}(y) d y=1
$$

where $\rho$ is the particle distribution in either the vertical or the horizontal plane. We have considered the luminosity equivalent beam size rather than the RMS beam size, since it de-emphasizes the contribution of particles far from the beam core. The tracked beam size versus the energy spread is shown in Fig. 3.5. The bandwidth needs to be compared with the nominal energy spread of the ATF extracted beam $\sim 0.08 \%$. As we see, both TURTLE and SAD tracking results show rather wide bandwidth in the vertical plane, while tracking with SAD shows a larger increase in the horizontal beam sizes.
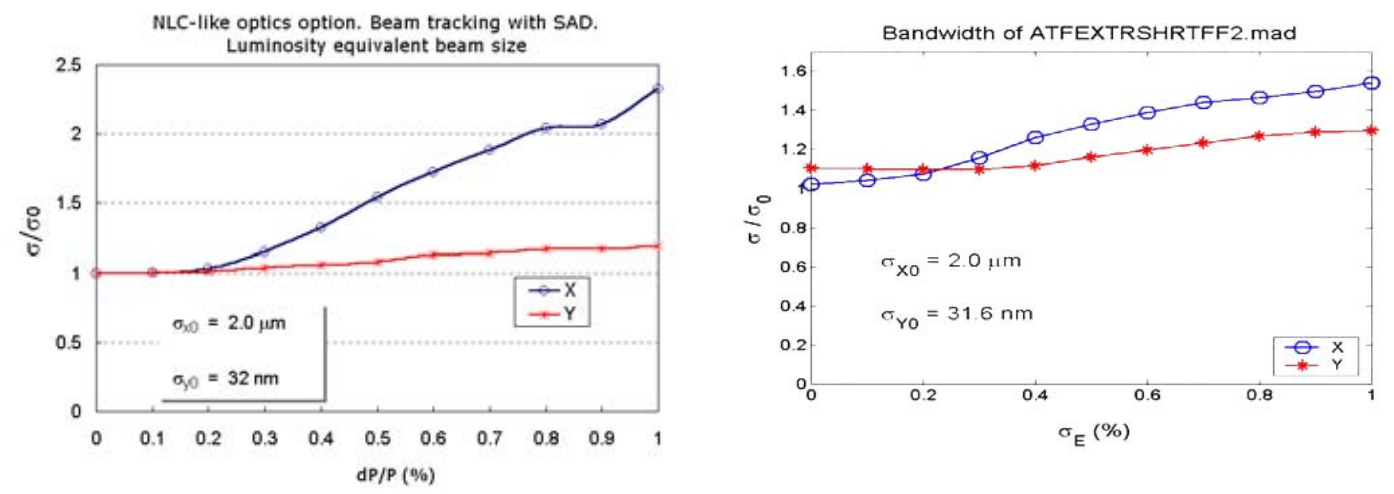

Figure 3.5: Bandwidth of proposed ATF2 optics computed with tracking by SAD (left) and TURTLE (right), with which it was optimized.

\subsubsection{Sensitivity to errors in ATF2 and ILC-FF}

One of the big challenges of producing stable colliding beams in ILC is maintaining the tight position and strength tolerances of the focusing elements and bending magnets. The sensitivity to errors in the ATF2 has been compared with the ILC final focus optics. This evaluation was performed analytically using FFADA program [13] with the beam parameters from Table 3.1.

The comparison of error sensitivities is shown in Fig. 3.6 and Fig. 3.7. As expected, the magnet tilt errors, driven by the beam size ratios, are very similar in ILC and ATF2. The position error sensitivities are relaxed by about a factor of five at ATF2, since the IP beam size is correspondingly larger, however, one must take into account that vibration amplitudes and motion caused by temperature variations are expected to be larger in ATF2 than in the underground ILC. The magnet strength error sensitivities are about the same in ATF2 and ILC for the Final Doublet, and about a factor of two tighter for other magnets, because the shorter ATF2 optics requires relatively stronger magnet fields.

Overall, the sensitivity to errors, and difficulty of achieving them, are similar in ATF2 and ILC. Thus the ATF2 will give valuable experience in providing stable beams for ILC. 


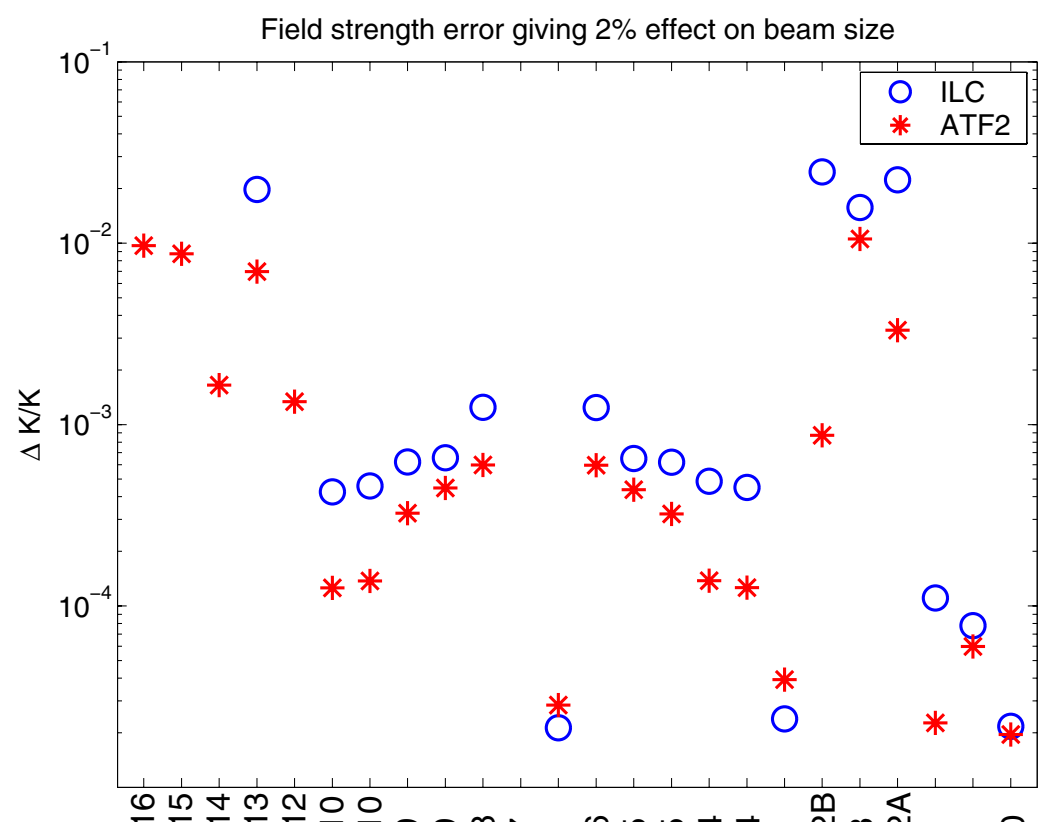

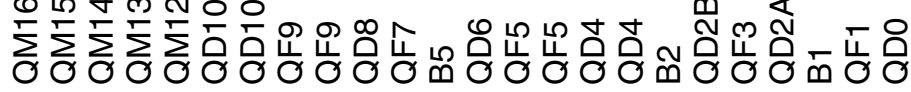

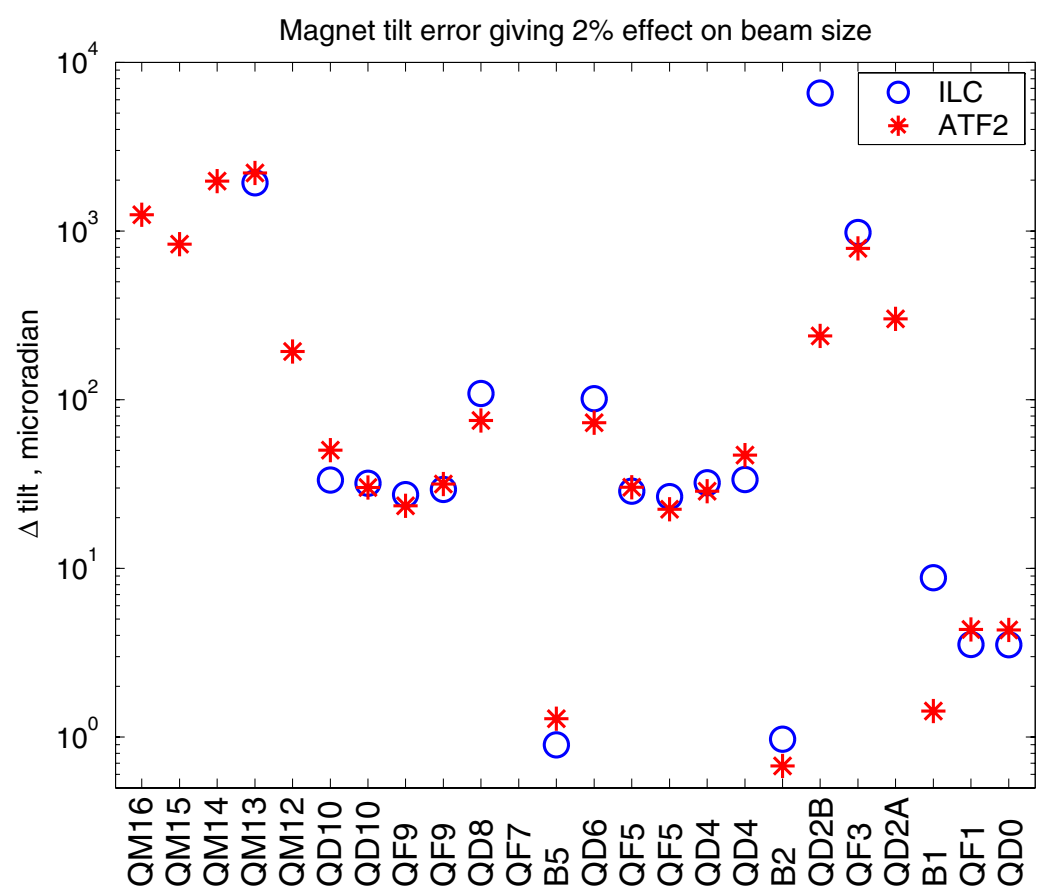

Figure 3.6: Field strength error (top plot) and magnet tilt error (bottom plot) giving $2 \%$ effect on beam size. Comparison of ATF2 optics and ILC optics. 


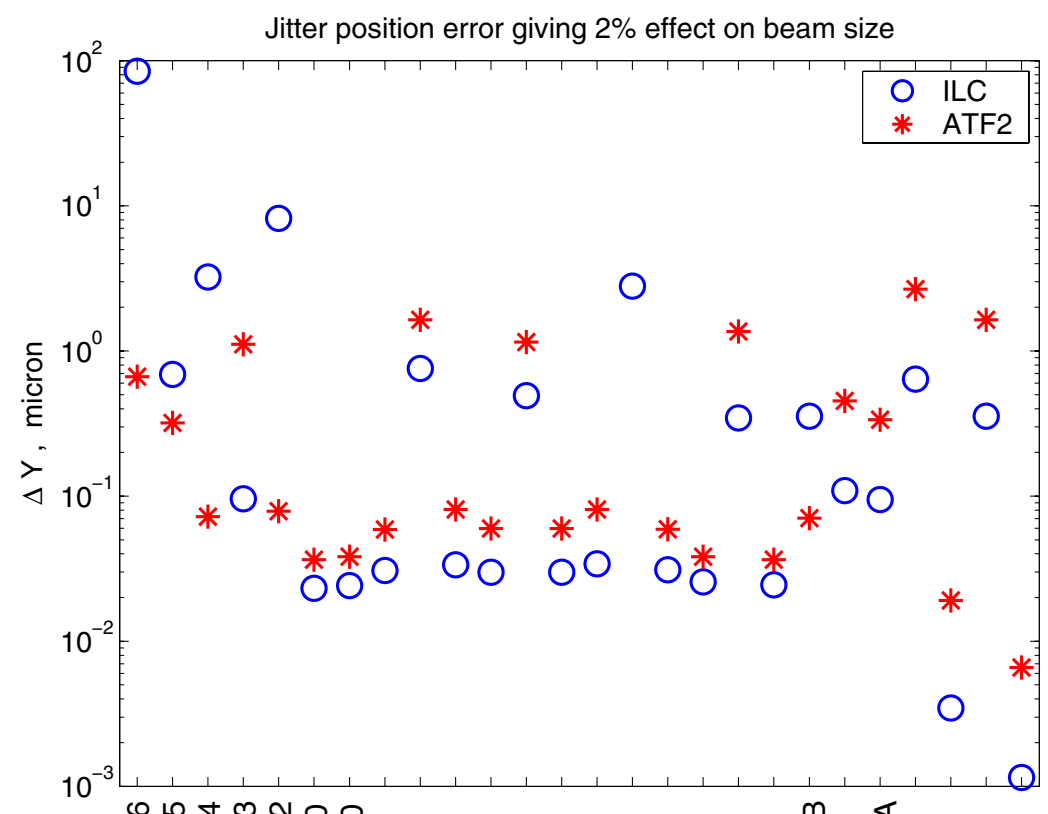

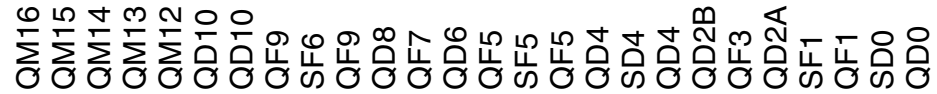

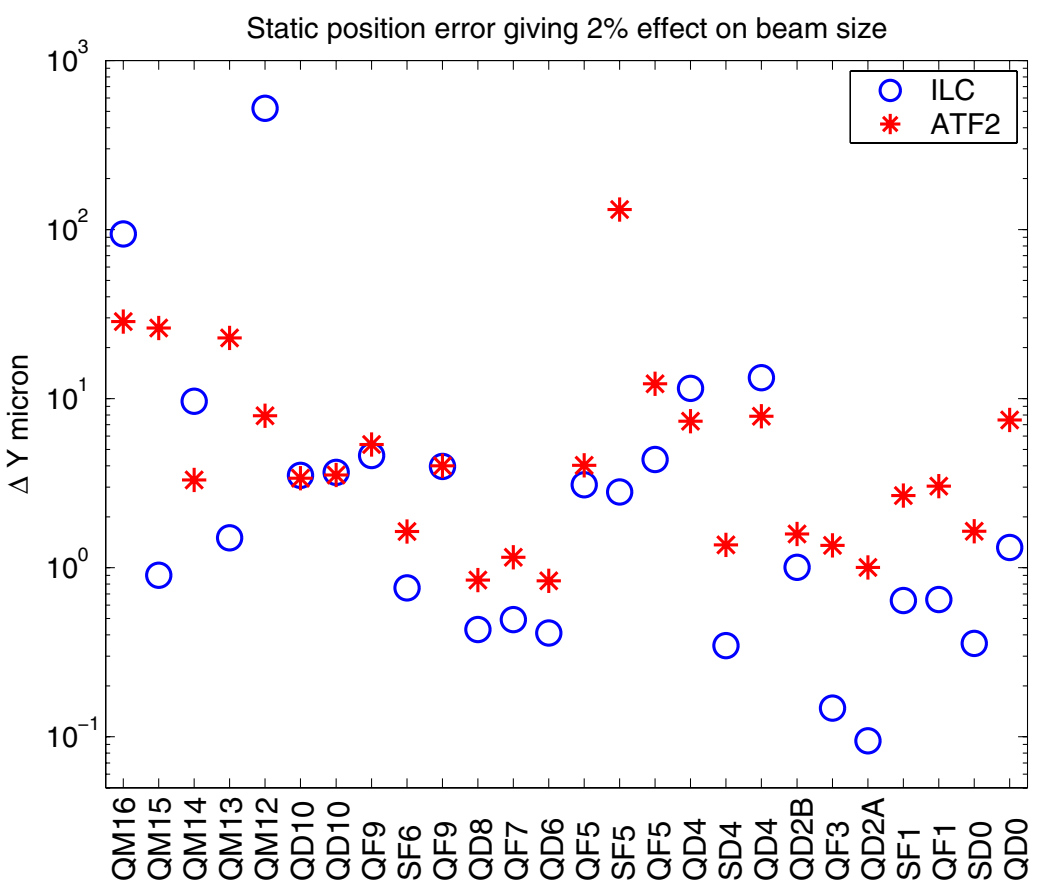

Figure 3.7: Jitter position error (top plot) static position error (bottom plot) giving $2 \%$ effect on beam size. Comparison of ATF2 optics and ILC optics. 


\subsection{Tolerances and tuneability}

In this section we describe simulations of orbit correction and tuning procedures for ATF2, which should give us estimates of the jitter, fast and slow errors for magnet position, rolls, strength errors and field shape errors. These results are preliminary and final tolerance specifications will be developed and published later this year in a separate document. Since the ATF2 simulations are not yet complete, we also give an example of earlier simulations for the NLC BDS optics.

\subsubsection{Tolerances}

The tolerances for the different magnet families are investigated to give guidance on the allowable jitter of magnets in terms of both position and field error, or more correctly the stability of the related power supply. These tolerances are given as an rms error that leads to either a $2 \%$ increase in beam size, or a change in beam position equivalent to $15 \%$ of the beam size; whichever leads to a tighter tolerance. The tolerances are divided into 3 regimes: errors that occur on timescales where they are effectively uncorrectable (hereon called Jitter), errors that can be corrected by the fast feedback correction system (fast errors), and those that can be tuned out using tuning algorithms (slow errors). These three regimes are obviously related to different time regimes, but these depend strongly on such factors as the bunch repetition rate and the latency in the correction algorithm and thus vary with the operating conditions of the machine. It is therefore conceivable that in one regime a tolerance is very loose, while in another it represents a major limitation on the system. In all three regimes the tolerances will be given for the main magnet families (quadrupole and sextupole) for both transverse position and power supply stability. Roll angle tolerances will also be given. The tolerances are divided into 4 different types or regimes:

1. Jitter is motion that occurs on timescales faster than the correction system. These errors can derive from a variety of sources such as cooling water supplies and fast ground motion.

2. Fast errors occur on timescales where the trajectory feedback system can be used to correct them. This is therefore related to the latency of the correction system as well as the inter-bunch spacing (actually, generally whichever is larger). To fully understand the tolerances for this form of error thus requires a design for the trajectory correction system. At the time of writing this had yet to be designed, and thus a representative system was created and used to understand the magnet tolerances. The correction system employs a corrector and BPM housed in every quadrupole of the FF line, except the final doublet, and is intended only as a conceptual design, with no basis in the final engineering reality.

3. Slow errors occur on timescales very much longer than the trajectory feedback system, and can include alignment errors that must be corrected before the machine can operate. These sorts of errors are generally corrected using specialized tuning algorithms, as well as the trajectory correction system.

4. All magnets in the final focus line will have error multipole components other than the design component. These may be random errors, or static errors arising from the magnet design. In 
effect these are equivalent in terms of the tolerance specification. No correction is performed when determining the field error tolerance.

\subsubsection{Tuning Algorithms}

To minimize the effects of errors, the ATF2 final focus requires a tuning procedure. The tuning algorithm can use both magnetic and mechanical means to restore the beam quality. At present, two methods of tuning the final focus are being studied.

1. The linear beam properties under error conditions are compared to the beam in perfect conditions. From this, a 6x6 "beam" matrix can be produced. The tuning procedure then involves minimizing this matrix, thus restoring nominal beam parameters. Individual "tuning knobs" are thus created consisting of 2 or 3 magnets, each of which modifies only one of the 36 " $\mathrm{R}$ "-matrix terms and which are applied in unison.

2. The measurable beam properties are scanned or analytically calculated and orthogonal tuning knobs are then created. This method benefits strongly from a lattice designed with such tuning knobs in mind. The tuning knobs can then be controlled by some automated system, or manually adjusted.

The first method only corrects the linear beam properties, though it may do this in a non-linear manner. This method also explicitly relies on the determination of the statistical distribution of the error beam, which may not be easily achieved experimentally. The second method has been extensively studied, for instance on the SLC and for the FFTB. This method benefits from a more direct analogy with well understood beam properties, but is harder to implement in a more generalized sense, requiring more direct user intervention. It does have the major advantage that the only beam properties that are corrected are those that are (believed) to be important.

\subsubsection{Beam Matrix Method}

This algorithm is based on inversion of the global "beam"-matrix. It is initially assumed for the purposes of simulation that all magnets in the final focus will be on X-Y translation stages. The response matrix thus uses the field strengths of all quadrupoles and sextupoles as well as two transverse motions of the magnets. The response matrix is generated initially by tracking several thousand particles along a perfect model of the FFS line and the 6 dimensional beam properties recorded at the IP. The individual magnets are then varied and the resulting IP beam properties again recorded. Using the beam-matrices generated, it is then possible to linearly solve the set of equations to form sets of orthogonal tuning knobs that affect only one aspect of the beam-matrix. To analyze the effectiveness of the tuning algorithm the final focus line is modeled with errors on all major magnets. Using a generalized Brent's method algorithm the tuning knobs are applied in order to try and minimize the resulting beam-matrix. Results for an example case, by no means the best or worse, are shown in Fig. 3.8. 

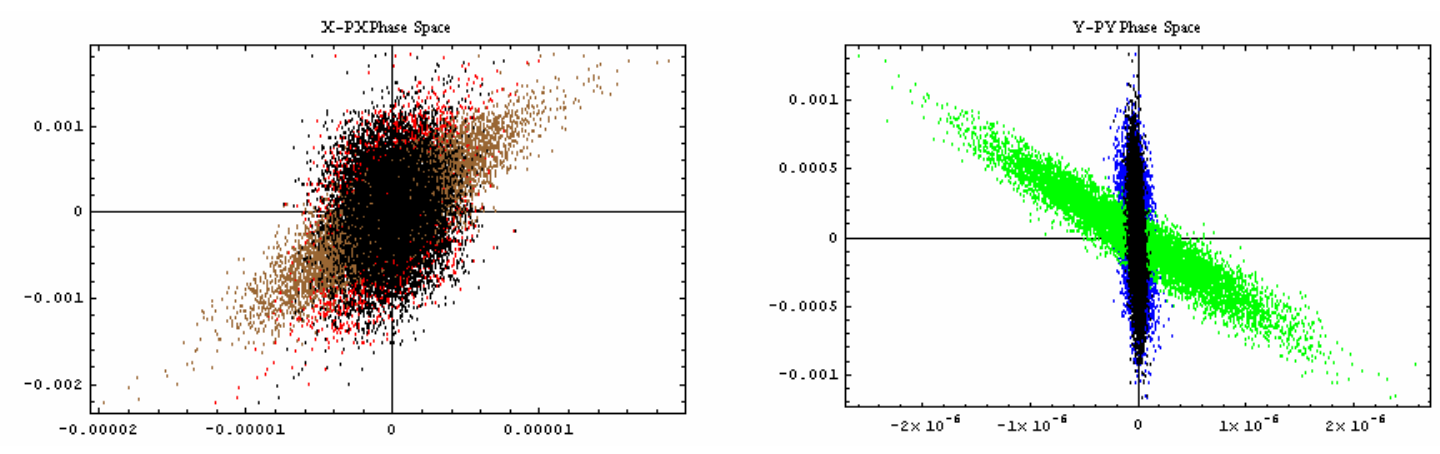

Figure 3.8: Results from simulation of the effectiveness of R-matrix tuning knobs at restoring the nominal beam parameters under random error conditions (Horizontally: brown = error beam, red $=$ corrected beam, black =nominal beam. Vertically: green $=$ error beam, blue $=$ corrected beam, black $=$ nominal beam).

\subsubsection{Analysis of the FFS using traditional methods}

The creation of tuning knobs using beam observables and/or well known beam parameters, such as the vertical dispersion, has been studied extensively at other accelerators. The basic method is the calculation, experimentally or analytically, of a response matrix of beam parameters with changes in some magnet property. From this response matrix orthogonal tuning knobs can be created that are used to correct the beams profile at the IP. Linear tuning knobs are made from feed-down effects in sextupole magnets. Generally orthogonal knobs are constructed using 3 or more magnets.

The tuning knobs investigated include $\beta_{x, y}$ shift and vertical dispersion. Tuning knobs for higher order terms have also been investigated, but this requires knowledge of the dominant higher order terms at the IP. To analyze these higher order terms simulations were performed with a T-matrix transformation at the IP, and over all T-matrix elements. The resulting change in beam size is then used to weight the various terms in order of importance. However, terms, which have a large effect on the beam size, are not necessarily those terms that are excited in the beam line under error conditions. This data was therefore convoluted with a second simulation that analyzed the change in matrix terms at the IP under error conditions. From the analysis the following higher order tuning knobs were created using the 5 final focus line sextupoles. The variable parameter (rotation of sextupoles $-\Delta$ roll, their strength change - delta $\mathrm{K} 2 \Delta \mathrm{K} 2$ ) is given in brackets:

T124 T322 T326 T344 ( $\Delta$ roll)

T122 T126 T166 T346 T324 ( $\Delta \mathrm{K} 2)$.

To analyze the effectiveness of the tuning algorithms, the ATF2 FF line is modeled with a selection of random errors on all magnet families. The magnitude of the error is then increased until the beam size increase is greater than $15 \%$ in both planes. This data is then interpolated and a tolerance deduced. Errors are applied only to magnets in the FF line, and not the extraction line, to decouple the possible error sources. 


\subsubsection{Tolerance Specification}

\section{Position Jitter}

The jitter tolerances are derived by applying random errors to all of the magnets and recording the RMS beam sizes. Tolerances are presented for errors in both transverse planes, as well as roll angle tolerances, see Table 3.2.

Table 3.2: Jitter tolerance specification.

\begin{tabular}{llll}
\hline & Horizontal & Vertical & Roll \\
\hline Quadrupoles & $<580 \mathrm{~nm}$ & $<2.7 \mathrm{~nm}$ & $<1.5 \mu \mathrm{rad}$ \\
Sextupoles & $<7.4 \mu \mathrm{m}$ & $<0.31 \mu \mathrm{m}$ & $<130 \mu \mathrm{rad}$ \\
\hline
\end{tabular}

\section{Fast Position Errors}

The fast error tolerances are again derived by applying random errors to all of the magnets and recording the beam size, see Table 3.3. 3 iterations of the SVD based correction algorithm were applied for each random seed, though no effort was made to minimize any increase in dispersion along the line.

Table 3.3: Fast error tolerance specification.

\begin{tabular}{llll}
\hline & Horizontal & Vertical & Roll \\
\hline Quadrupoles & $<580 \mathrm{~nm}$ & $<3.0 \mathrm{~nm}$ & $<1.5 \mu \mathrm{rad}$ \\
Sextupoles & $<15 \mu \mathrm{m}$ & $<0.76 \mu \mathrm{m}$ & $<332 \mu \mathrm{rad}$ \\
\hline
\end{tabular}

Note that if the orbit correction feedback were properly configured (in particular, to hold the orbit fixed in the sextupoles), the tolerances for fast errors would be significantly relaxed in comparison with jitter tolerances. This is not the case yet, and indicates that the configuration of BPM/correctors need to be improved.

If one assumes that the correction system is perfect and looks only at the effects from the increase in beam size, the tolerances in Table 3.4 are applicable.

Table 3.4: Fast error tolerance specification.

\begin{tabular}{llll}
\hline & Horizontal & Vertical & Roll \\
\hline Quadrupoles & $<880 \mathrm{~nm}$ & $<230.0 \mathrm{~nm}$ & $<1.5 \mu \mathrm{rad}$ \\
\hline
\end{tabular}

\section{Tunable Position Errors}

The tolerances for tunable errors were calculated using the traditional tuning method. The tuning 
knobs were applied in order from Beta waist shifts through the various higher order knobs. 1 iteration of the correction system was applied at the beginning of each tuning iteration. 2 tuning iterations were performed for each random error. The dispersion tuning knobs were not found to be useful when the line contained errors and so was not applied. The tolerances are shown in Table 3.5.

Table 3.5: Tunable errors tolerance specification.

\begin{tabular}{llll}
\hline & Horizontal & Vertical & Roll \\
\hline Quadrupoles & $<79 \mu \mathrm{m}$ & $<0.14 \mu \mathrm{m}$ & $<1.2 \mu \mathrm{rad}$ \\
\hline
\end{tabular}

Note again, with properly configured orbit correction feedback and knobs, the slow error tolerances can be even more relaxed. The position tolerances indeed became looser, but not the roll angle tolerance. This tells that the present configuration need to be improved.

Since these simulations for ATF2 optics were not finished at the time of writing this proposal, an example of earlier NLC simulations will be given further below.

\section{Field Errors}

Field error tolerances for extraction line magnets are given in two scenarios:

1. Tolerances are derived for each individual magnet and for each multipole order.

2. Tolerances are derived with all magnets having errors and for all multipole orders. The magnitude of the multipole error is proportional to the $b_{2} / b_{3}$ of the relevant magnet.

The minimum absolute values of $B_{n} / B_{2}$ at $\mathrm{r}=1 \mathrm{~cm}$ for all of the magnets is shown in Fig. 3.9 (left). Table 3.6, and Fig. 3.9 (right) give the relative values of $B_{n} / B_{2}$ for all quadrupoles, where the amplitude of the multipole component on each magnet is proportional to the strength of that quadrupole multiplied by the values in the table. Table 3.7 gives the relative multipole data $B_{n} / B_{3}$ for the sextupoles.
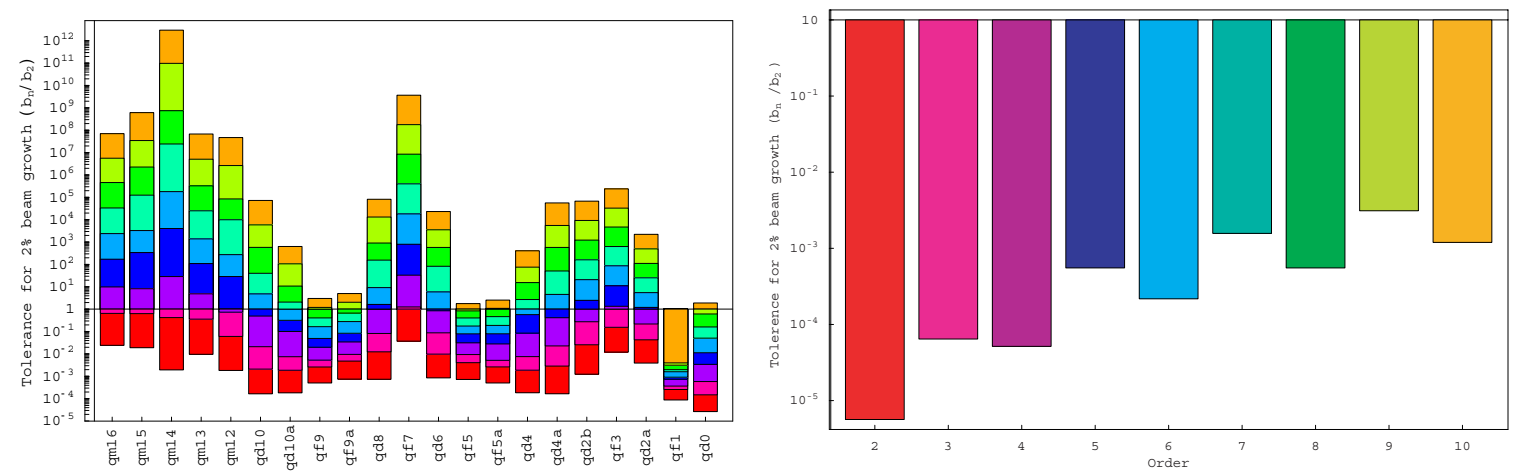

Figure 3.9: Tolerances on FF line quadrupoles in terms of individual quadrupoles (left) and as all quadrupoles together (right) and over all normal multipole orders. 
Table 3.6: Tolerance specifications for the quadrupole magnets.

\begin{tabular}{llllllllll}
\hline Order & 10 & 9 & 8 & 7 & 6 & 5 & 4 & 3 & 2 \\
\hline Normal $\left(10^{-4}\right)$ & 12.0 & 31.1 & 5.53 & 15.7 & 2.17 & 5.53 & 0.516 & 0.644 & 0.056 \\
Skew $\left(10^{-4}\right)$ & 4.41 & 2.66 & 2.21 & 1.27 & 0.941 & 0.507 & 0.253 & 0.117 & 0.017 \\
\hline
\end{tabular}

Table 3.7: Tolerance specifications for the Sextupole magnets.

\begin{tabular}{llllllllll}
\hline Order & 10 & 9 & 8 & 7 & 6 & 5 & 4 & 3 & 2 \\
\hline Normal & 0.247 & 0.306 & 0.105 & 0.126 & 0.0337 & 0.0353 & 0.0066 & 0.0093 & 0.00043 \\
Skew & 0.0591 & 0.0374 & 0.0268 & 0.0158 & 0.00974 & 0.00478 & 0.0021 & 0.000929 & 0.00012 \\
\hline
\end{tabular}

\subsubsection{Tuning example on NLC BDS}

Given that the tuning and orbit correction simulations for the ATF2 lattice are ongoing, and the procedures still need to be improved, we consider it useful to give here an example of earlier simulations of tuning for the NLC BDS lattice (which is similar to the present ATF2 optics, just longer). Fig. 3.10 shows horizontal and vertical beam sizes at IP during the orbit correction and tuning procedure. Simulations were performed by Mat-LIAR code using tracking of $40 \mathrm{~K}$ particles with the errors shown in the Table 3.8. The blue and green bins in Fig. 3.10 show 2 iterations of 17 knobs, where each "knob number" contain 2 steps: 1) knob and 2) orbit correction. The knobs order is the following: coupling, y-waist, x-waist, Dy, Dx, T322, T362, T366, T342, T346, T344, T122, T162, T166, U3422, V34222, V36422. The orbit correction was performed using 18/20 (x/y) quad movers and BPMs.
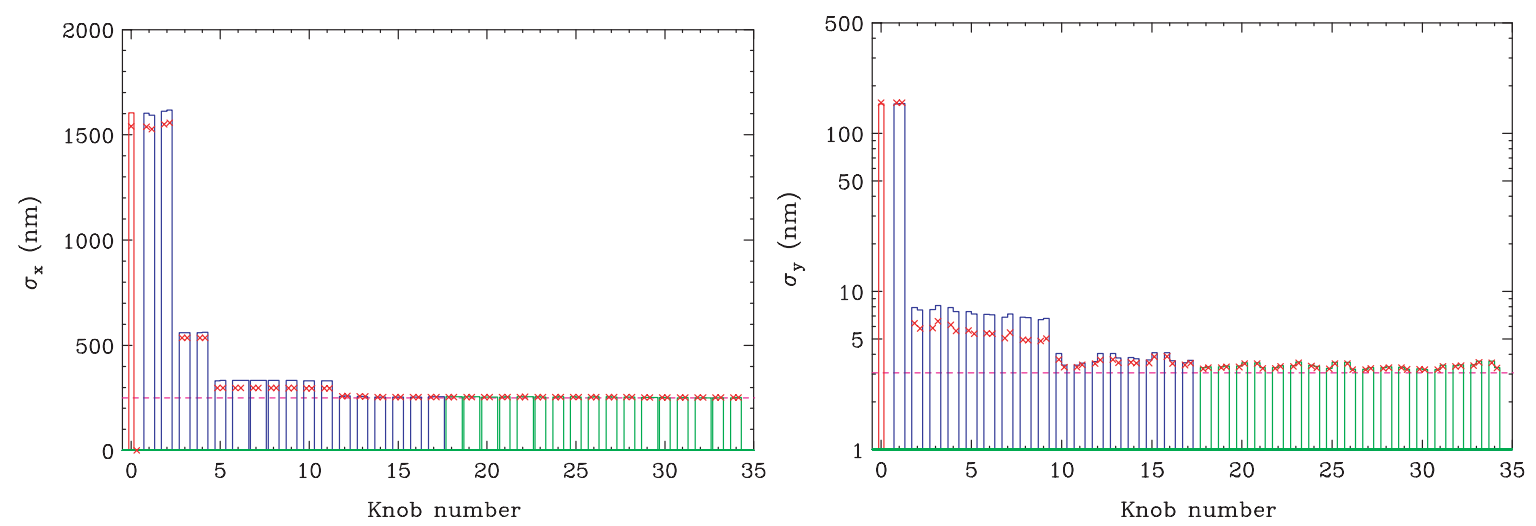

Figure 3.10: Tuning example for NLC BDS optics with errors shown in Table 3.8, showing horizontal and vertical beam sizes during the orbit correction and tuning procedure. The dash line shows the beam sizes without errors. See text for explanation of the knobs. The histogram bars show standard rms beam size for full beam and the $\mathrm{x}$ symbols show the gaussian fit sigma for the beam core.

These earlier simulations with the NLC optics have shown that the slow errors are quite relaxed. We 
Table 3.8: Errors used in tuning simulations of NLC BDS optics.

\begin{tabular}{llll}
\hline & $\mathrm{dK} / \mathrm{K}$ & tilt $(\mathrm{rad})$ & $\mathrm{dx} / \mathrm{dy}(\mu \mathrm{m})$ \\
\hline quads & $2.5 \mathrm{e}-3$ & $1 \mathrm{e}-4$ & $15 / 5$ \\
QF1, QD0 & $5 \mathrm{e}-4$ & $5 \mathrm{e}-5$ & $15 / 5$ \\
sextupoles & $1 \mathrm{e}-2$ & $3 \mathrm{e}-4$ & $15 / 5$ \\
octupoles & $2.5 \mathrm{e}-2$ & $1 \mathrm{e}-3$ & $15 / 5$ \\
decapoles & $5 \mathrm{e}-2$ & $1 \mathrm{e}-3$ & $15 / 5$ \\
\hline
\end{tabular}

expect tolerances for slow errors at the ATF2 to be similar or even somewhat looser (according to the ATF2/NLC error sensitivities shown in Fig. 3.6 and Fig. 3.7).

\subsection{Beam Diagnosis}

In this section, beam diagnosis in the ATF2 is briefly described.

\subsubsection{Twiss Parameters and Emittance at the Entrance of Final Focus(FF) Line}

In the downstream end of the ATF extraction line, there are five wire scanners (WSs), that can measure $\alpha, \beta$ and $\epsilon$ there. The region was designed to be dispersion free and the phase advances of these WSs are almost 45 degrees in both of $x$ and $y$ directions. Each WS consists of five wires; horizontal, vertical, 45 degree and \pm 10 degree wires. The wire is made of tungsten/carbon with diameter of $10 / 7 \mu \mathrm{m}$, while the beam size there is about $100 / 10 \mu \mathrm{m}$ in the horizontal/vertical direction, respectively. An example of the WS signal is shown in Figure 3.11. In reality there is dispersion and we need to correct it before measurement. Usually the correction is done until the vertical dispersion is less than $10 \mathrm{~mm}$, contribution of which to the beam size is less than $10 \mu \mathrm{m}$ assuming $d p / p=10^{-3}$. Then measured data is analyzed considering the effect of dispersion and wire size. An example of the analysis result is shown in Figure 3.12. The WSs can also measure $x-y$ coupling of the beam motion with 45 degree wires, though in design, those motions are decoupled. With these measured parameters, the optics can be matched using matching quadrupole magnets upstream of the FF line.

The wire scanner measurement is invasive to the beam operation. R\&D efforts for less invasive laserwire scanners have recently been initiated with the goal of eventually replacing the present tungten/carbon wires. Section 5.2 discusses the design and operation of an improved diagnostics section in the extraction line.

\subsubsection{Beam Orbit}

Beam position monitors (BPM) are needed to transport the beam through to the beam dump. They are also required to measure and correct the beam orbit in order to achieve the design beam size at 


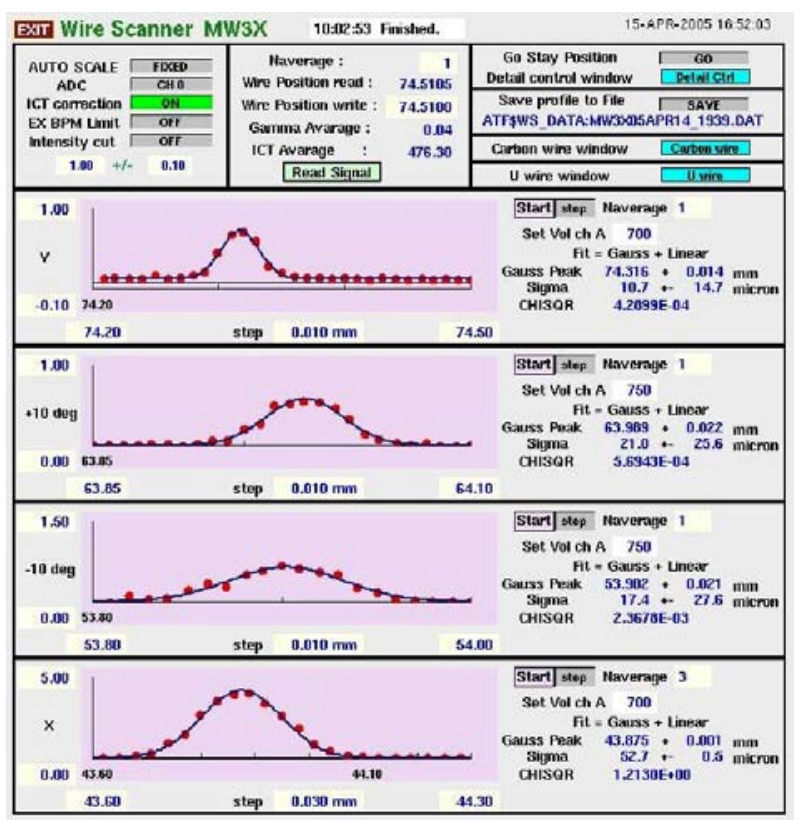

Figure 3.11: An example of WS signals. From top to bottom, signals from $y,+10 \mathrm{deg},-10 \mathrm{deg}$, and $x$ wire are shown.

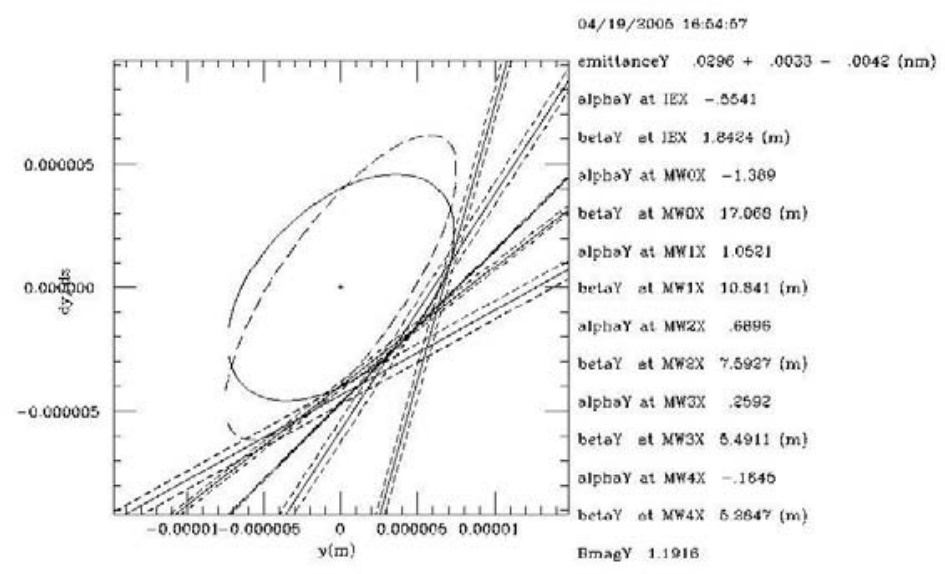

Figure 3.12: An example of WS analysis. Five WSs are used in this analysis. 
the IP. Cavity type BPMs are foreseen for ATF2 with resolution assumed to be $100 \mathrm{~nm}$. The cavity BPMs are attached to all the quadrupole and sextupole magnets in the final focus line. Each magnet will be supported by an $\mathrm{x}-\mathrm{y}$ mover used to correct the measured beam orbit.

\subsubsection{Beam Size at IP}

The first goal of the ATF2 is to achieve a very small beam size at the IP, so a beam size monitor is needed. The Shintake monitor used at FFTB will be installed at the ATF2 IP. The design vertical beam size is about $30 \mathrm{~nm}$, and the beam jitter is assumed to be less than $30 \%$ of the beam size. The resolution of the monitor must be less than $10 \mathrm{~nm}$ in order to measure the beam size to less than $40 \mathrm{~nm}$. This monitor will be used also in beam tuning, where a shorter measurement time is preferable.

\subsubsection{Cavity BPM at IP}

The second goal of the ATF2 is to stabilize the beam to the nanometer level. In this phase, a cavity BPM will be installed at the IP instead of the beam size monitor. This BPM must have a position resolution of $2 \mathrm{~nm}$ and will also be used for a beam feedback system.

\subsubsection{Other Monitors}

At the start of ATF2 commissioning, the beam must be transported through to the beam dump. At this stage, it is better to have beam position monitors with established performance and high resolution is not required. They will not be attached to all of the quadrupole magnets,and thus they could be stripline BPMs or screen monitors. The latter can also measure the beam profile to check the optics of the FF line. When the measured beam profile is much different from one expected from the design optics, we can see there is something wrong in the real optics. In that sense, screen monitors can be used to check the optics of the FF line.

In case we introduce some bunch compression, bunch length monitor will be required. The bunch compression can be done adding a bunch compressor line or using RF technique in the damping ring. Anyway it is an option for the ATF2; thus the bunch length monitor is also optional. As the monitor, an optical diffraction radiation (ODR) monitor will be employed. It is now being studied at ATF. 


\section{Instrumentation}

\subsection{Cavity BPMs}

Two types of beam position monitors are required, namely Q-BPM and IP-BPM. Cavity BPM technology will be used for both types, but with different designs. The salient features of a cavity BPM are the accuracy of its center position and the possibility to reach high resolution. The cavity BPM measures an RF excitation in a cavity induced by a beam. The amplitude of the lowest transverse dipole mode is proportional to the beam position and its charge. When the beam passes through the center of the cavity, no dipole modes are excited. The electrical center is determined only by the structure, and is expected to be stable during operation. A high gain RF circuit readout will provide the ultimate resolution, though the range will be limited due to saturation of the electronics.

The Q-BPMs monitor the beam position at the quadrupole magnets in order to maintain the orbit in the center of the magnetic field, and avoid undesireable kicks, which would limit the achievable beam size at the interaction point. They require reasonable resolution and good center accuracy. The IP-BPM is placed at the focal point to measure the transverse beam stability, which is one of the most important achievements of part $\mathbf{B}$ of the ATF2 project. The goal is to stabilize the vertical beam position to within less than a few nm. A specially designed ultra high resolution beam position monitor is necessary to monitor the beam position to sufficient precision.

\subsubsection{Q-BPM}

A high resolution beam position monitor is rigidly attached to each quadrupole magnet (as shown in Figure 4.1). The resolution of a single pass measurement must be better than $100 \mathrm{~nm}$. The accuracy (mechanical and electronical stability during operation) is required to be better than $1 \mu \mathrm{m}$.

The structure of the cavity BPM is illustrated in Figure 4.2. It consists of a sensor cavity of pill-box shape and four waveguides. The cavity and the waveguides are connected with slots placed on the end plate of the cavity. The dipole mode signal in the sensor cavity is selectively read out to the waveguides through slots via magnetic coupling. Then antennas on the waveguides pick up the signal into coaxial cables.

The detection electronics is shown in Figure 4.3. First, signals from opposite ports are combined with a 180 degree hybrid. This increases the dipole signal and suppresses the common mode components at the same time. The signal is then fed into a front-end electronics box. A single stage mixer downconverts the RF signal to $20 \mathrm{MHz}$ frequency. Then, it is delivered to a recorder placed outside the tunnel. In order to suppress unwanted sidebands, an image rejection mixer is used. A cw source synchronized to the beam is used as a local oscillator for the mixer, to eliminate phase ambiguity. $14 \mathrm{bit}, 119 \mathrm{MHz}$ digitizers record the wave form. An online analysis procedure fits the waveform and 


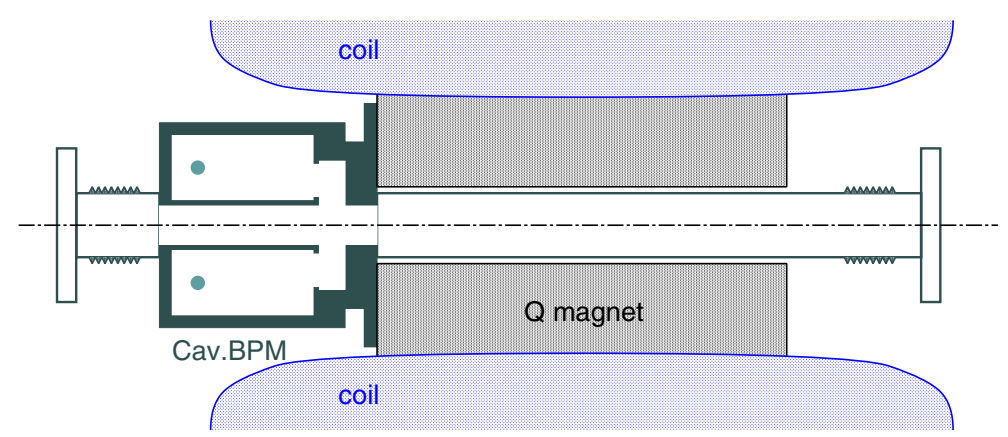

Figure 4.1: Cavity BPM attached on a quadrupole magnet.

extracts the beam positions. The noise limited resolution is estimated to be $30 \mathrm{~nm}$.

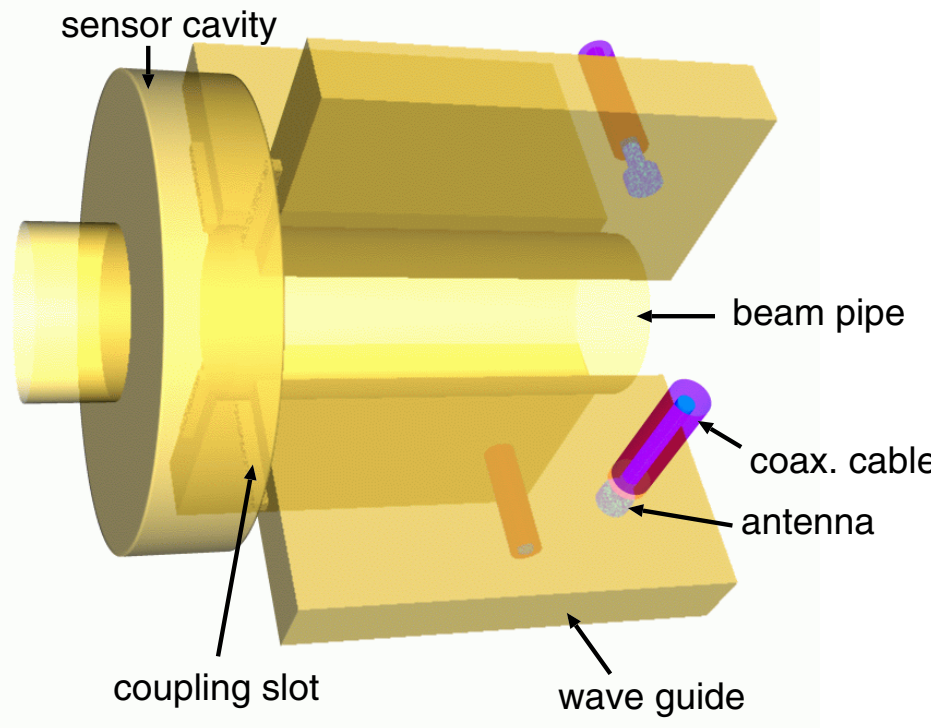

Figure 4.2: Structure of the Q-BPM.

The dynamic range is expected to be approximately $500 \mu \mathrm{m}$, which is determined by saturation of the electronics. The electrical center of the BPM and the field center of the magnet should be aligned to much better than this. In the first month of the commissioning, we may need additional attenuators at the input of the electronics in order to extend the range. A beam-based method will be used to determine the offsets of the BPMs with respect to the field center of the magnets. During a beam shut-down period, the position of the BPMs can be finely adjusted by the measured offsets. Then, the attenuators can be removed to have maximum sensitivity. 


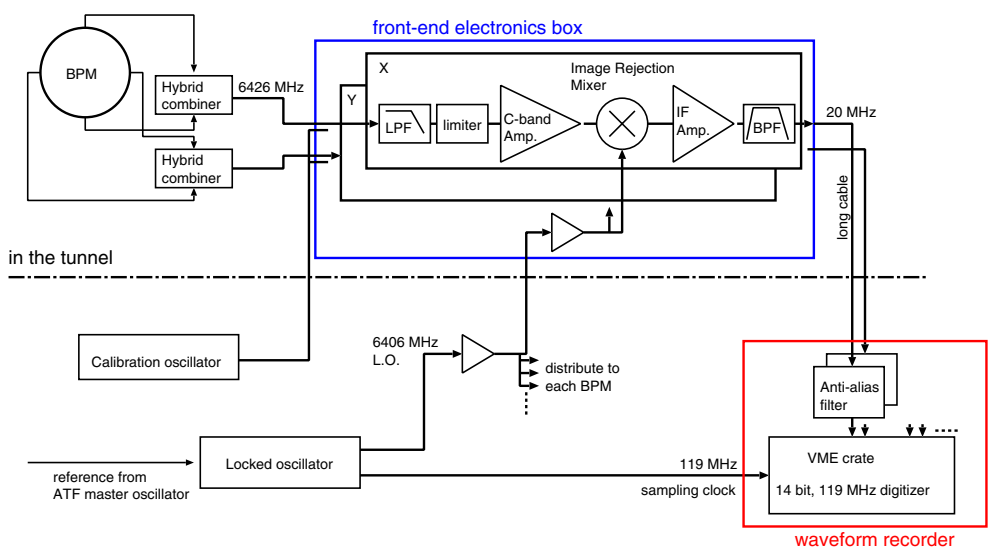

Figure 4.3: Electronics of the Q-BPM.

The BPM calibration will be done by using the movers of the magnet to which it is rigidly attached. The gain stability of the electronics will be routinely monitored using a test signal generated by an external oscillator.

\subsubsection{IP-BPM}

The IP-BPM is required to have the best possible resolution in order to measure position jitter to a few nanometers in the vertical plane. Special designs are needed to suppress effects which limit the resolution, such as coupling from the horizontal beam position and contamination from the beam angle signal.

Because the beam jitter usually scales as the beam size, the beam is expected to have much larger jitter in the horizontal plane than in the vertical plane. In order to measure the small beam jitter in the vertical plane, it is critical to suppress crosstalk from the horizontal position information. Even if the cavity shape is designed to be symmetric around the beam axis, imperfections in the fabrication easily mix the information from the two planes. One solution to achieve good isolation between the planes is to introduce a large frequency difference between the dipole modes of different polarization. One possibility is to place posts on one plane to significantly shift the frequency (Figure 4.4). A frequency difference of $1 \mathrm{GHz}$ is expected to produce $-80 \mathrm{~dB}$ isolation between planes.

Since the final focus optics converts beam position jitter at its input into angle jitter at the intraction point, the IP-BPM has to work well even with large angle jitter. An angled trajectory also excites the dipole mode in the cavity BPM, but out of phase with respect to the position signal. Although phase detection helps to suppress the angle signal, further reduction is necessary. In order to reduce the sensitivity to the angle, the effective cavity length is designed to be small. This modification also reduces the position sensitivity which must be compensated by reducing the diameter of the beam pipe. The position resolution is expected to be $1 \sim 2 \mathrm{~nm}$, while suppressing the sensitivity to beam angle to better than $200 \mu \mathrm{rad}$. 


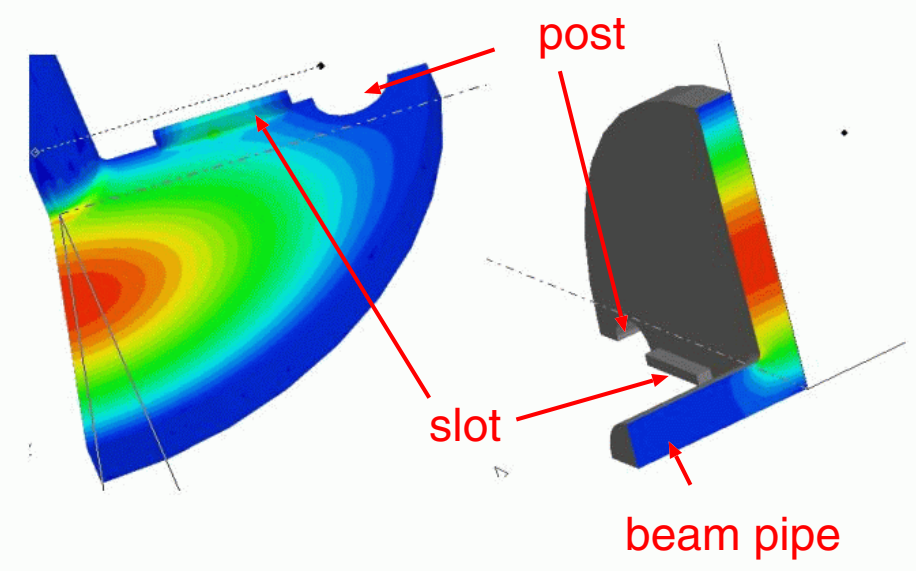

Figure 4.4: Electric field of the dipole mode in the IP-BPM.

Figure 4.5 shows the layout of the IP-BPM. A triplet of BPMs is used to measure the beam trajectory with high precision. The center BPM is placed at the focal point. A reference cavity will be used that operates in symmetrical modes (not shown in the figure). This cavity measures the beam charge, the bunch length and the beam angle using TM010, TM020 and TE011 mode, respectively.

The electronics is shown in Figure 4.6. The transient signal on the leading edge of the pulse is rejected by an RF switch. A two-stage synchronous detection scheme is used to reduce the overall bandwidth. The local oscillator for the first down conversion mixer is produced from a signal from the reference cavity. In the second stage, the signal is rectified into two parts, the in-phase component which is position sensitive and the out-of-phase component which is angle sensitive. Both are recorded together with other useful quantities from the reference cavity.

\subsection{Wakefield effects due to Cavity BPMs}

To calculate the wakefields from the cavity BPM's, we begin with a diagram of their geometry shown in Fig. 4.5. As shown on the sketch, the total length of $3 \mathrm{BPM} /$ bellows/flange combinations (which we call 3 BPM modules) is equal to $186.5 \mathrm{~mm}$. The rest of the dimensions were scaled from the sketch, and then the iris radius was reduced from $3 \mathrm{~mm}$ to $2.5 \mathrm{~mm}$, according to the latest design. The flange gap was assumed to be $4 \mathrm{~mm}$ (which may be pessimistic). In the real design, the BPM $x$ and $y$ mode frequencies differ, thus there must be broken symmetry in the geometry; for calculational purposes we use a cylindrically symmetric model. We believe that these assumptions are reasonable for the required accuracy of our estimations. The geometry of one BPM unit of our model is shown in Fig. 4.7. 


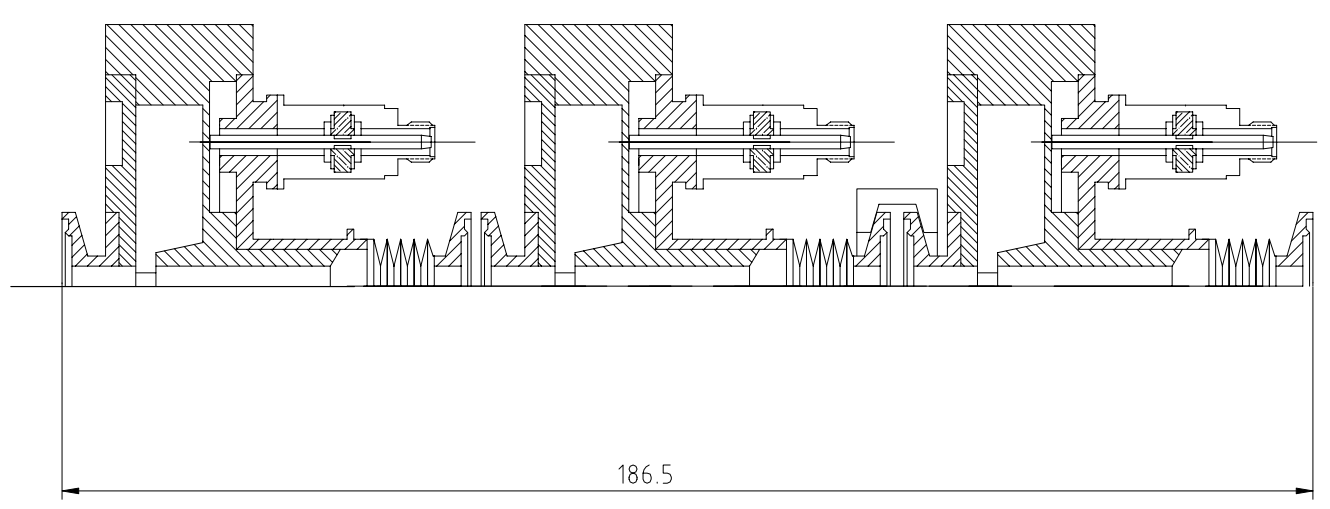

Figure 4.5: Layout of the IP-BPM.

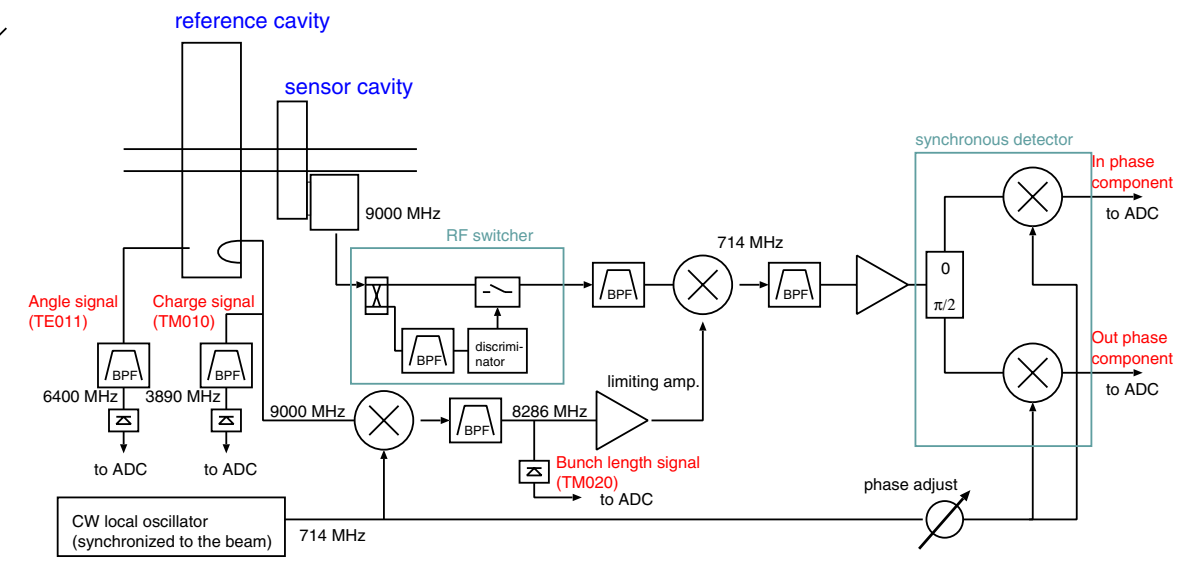

Figure 4.6: Electronics for IP-BPM.

To obtain the short-range, dipole wakefield of the cavity BPM's we use the $2 \mathrm{~d}$ version of MAFIA. The bunch is assumed to be gaussian with rms length $\sigma_{z}=8 \mathrm{~mm}$. The wake for one BPM segment is shown in Fig. 4.8 (the dashed curve gives the bunch shape). The wake is resistive (also gaussian in shape); the average is $\left\langle W_{x}\right\rangle=1.16 \mathrm{~V} / \mathrm{pC} / \mathrm{mm}$. Breaking the module into its parts we obtain for the cavity/bellows/flange: $0.33 / 0.53 / 0.30 \mathrm{~V} / \mathrm{pC} / \mathrm{mm}$. Taking as bunch charge $Q=3 \mathrm{nC}$ and energy $E=1.3 \mathrm{GeV}$, we obtain average kick angles per unit offset, $\left(\Delta y_{w a k e}^{\prime} / \Delta y\right)$, for cavity/bellows/flange: $0.78 / 1.23 / 0.70 \mathrm{nr} / \mu \mathrm{m}$. Note that the peak kicks are $\sqrt{2}$ larger.

By kicking and spreading the beam out transversely, the wakefields affect the resolution of the monitors. Consider now that there are 6 BPM modules lined up near the interaction region, 3 for $x$ and 3 for $y$; there is a flange at the beginning and at the end. The longitudinal positions of the cavity/bellows/flange within a module are 10/50/60 mm. To model initial jitter offset in the beam, suppose the monitors are all transversely aligned, and the beam moves parallel to the axis at offset $1 \mu \mathrm{m}$. Summing all the wake kicks, the final angle is $6 \mathrm{nr}$ and final offset is $0.35 \mathrm{~nm}$, still small 


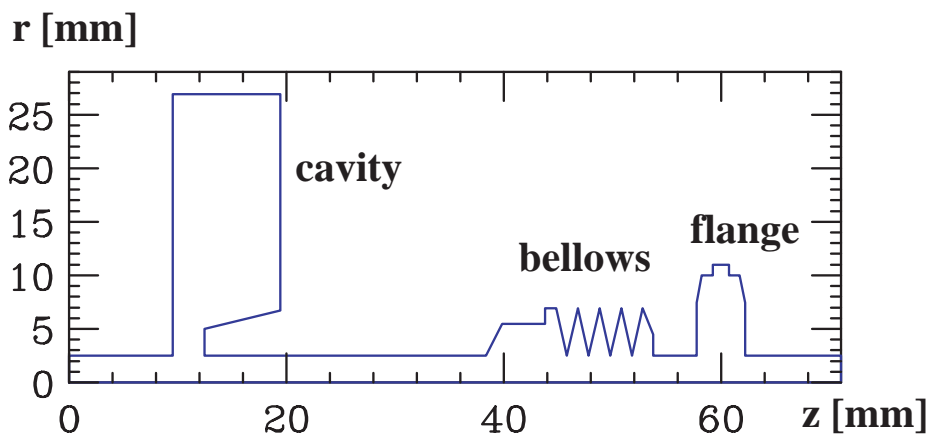

Figure 4.7: Geometry used for wakefield calculation.

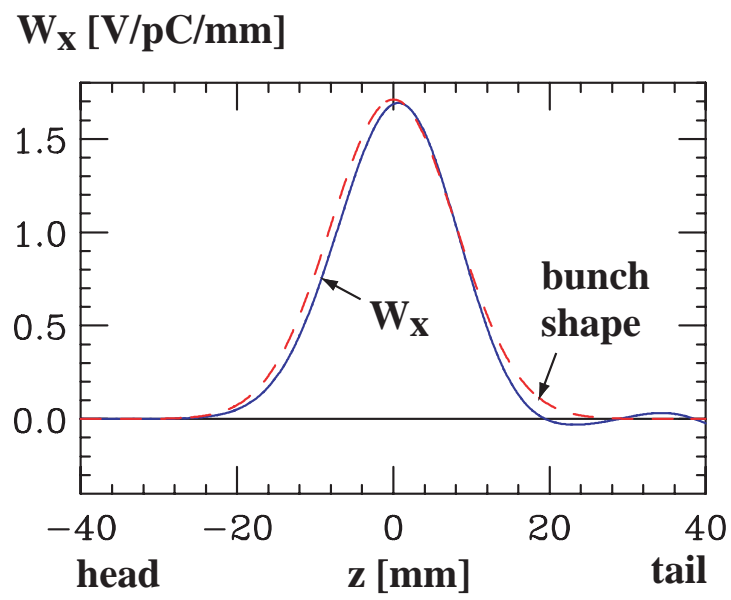

Figure 4.8: Transverse wake of one cavity BPM module. The bunch shape, with head to the left, is given by dashes. 
compared to a $y$ resolution of $2 \mathrm{~nm}$.

More difficult to achieve may be the angle jitter tolerance. To model this we consider a beam with initial angle and no offset. The extra offset due to the wake at element $n$ is given by

$$
\Delta y_{\text {wake }, n}=\alpha\left(\frac{\Delta y_{\text {wake }}^{\prime}}{\Delta y}\right) \sum_{i}^{n} z_{i}\left(z_{n}-z_{i}\right),
$$

with $\alpha$ the initial angle of the beam and $z_{i}$ the longitudinal location of element $i$. For $\alpha=10 \mu \mathrm{r}$, we find at the $i^{\text {th }}$ BPM cavity locations, the offsets $\left\{i, \Delta y_{\text {wake, } i}[\mathrm{~nm}]\right\}:\{1,0\},.\{2,0.02\},\{3,0.16\}$, $\{4,0.50\},\{5,1.16\},\{6,2.23\}$. To have the best resolution in $y$, we see that the monitors need to be arranged in the order $y-y-y-x-x-x$. Then, if we want $2 \mathrm{~nm}$ resolution in $y$ (25\% of the beam divergence), we see that about $125 \mu \mathrm{rad}$ of angle jitter is allowed.

These calculations have assumed that the beam enters the first BPM module unperturbed. At the moment we have used a tentative design of the BPM modules. The fact that we have used a cylindrically symmetric approximation to the BPM cavity probably does not affect significantly its short-range wakefield. If one would like to reduce the wake effect one can, in principle, redesign and reduce the impedance of the bellows and flanges; in this way one may be able to gain up to a factor of 3 in jitter tolerance. Finally note that, in our calculations, we have taken as bunch charge a conservative $3 \mathrm{nC}$, which is much larger than the nominal value of $0.5 \mathrm{nC}$.

\subsection{Laserwire}

Achieving the goal of a $37 \mathrm{~nm}$ spot-size at the ATF2 will require a detailed understanding of the beam properties before the final focus elements. At the ILC the high intensities and small electron beam sizes mean that the beam phase space will have to be be measured using laser-based beam diagnostics, namely laserwires for electron spot-sizes of order a few microns or more, and the "Shintake" monitor, described in Sec. 4.4.1 for electron spot-sizes of order a few 10s of nanometres.

The laserwire uses a finely focused laser beam to scan across the electron beam such that the resulting Compton scattered photons (or electrons) can be detected downstream and the rate of events determined as a function of relative position of electron and laser beams. A good knowledge of the laser beam spot size then allows the electron beam size to be determined.

The first use of a laserwire in an HEP experiment was accomplished at the SLD experiment [14], where micron laser spot-sizes were achieved using a laserwire system located near the IP of the SLD detector. More recently a laserwire based on a CW laser plus high-Q cavity has been used to good effect at the ATF ring [15], and a high-power pulsed laser system is operational at the PETRA ring [16].

\subsubsection{Requirements}

The ATF2 project will enable laserwires to be used in a very similar environment to that of the ILC. At the ILC it will be necessary to make a measurement of the electron bunch spot-sizes within a 
bunch train of about 3000 bunches, where the inter-bunch spacing is about $300 \mathrm{~ns}$. This "single shot" requirement necessitates the use of a high-power pulsed laser system, with good laser mode quality, both spatially and temporally, combined with ultra-fast scanning techniques.

The width $\sigma_{m}$ of the raw measured laserwire profile is an intricate convolution involving the laser spot size $w / 2=\sigma_{\ell}$, the Rayleigh range $z_{R}$ and the vertical-horizontal aspect ratio of the electron bunch, where:

$$
\begin{array}{r}
\sigma_{\ell}=\frac{\lambda f}{D} \\
z_{R}=\frac{4 \pi \sigma_{\ell}^{2}}{\lambda}
\end{array}
$$

$\lambda$ is the wavelength (in our case we propose to use green light at $\lambda=532 \mathrm{~nm}$ from an existing NdYag doubled laser), $f$ is the focal length and $D$ the diameter of the laser final focus optics. Some representative values of $\sigma_{\ell}$ are given in Table 4.1. In this table a laser $M^{2}=1.3$ is assumed, which is the typical value measured for the present ATF laserwire and $f \#$ is the optimal $f / D$ value for the given electron beam dimensions, including Rayleigh range effects. $\sigma_{\ell}$ is the laser spot-size at the waist and $P_{\ell}$ is the instantaneous laser power required to yield a $1 \%$ vertical electron spot-size measurement from five (Gaussian) scan points. Using the same laser pulses to scan the $x$-dimension would result in a $3.7 \%$ statistical error.

Table 4.1: Laser spot-sizes for green laser light of wavelength $532 \mathrm{~nm}$ and optimised laser optics, assuming an electron-bunch aspect ratio $\sigma_{x}^{e} / \sigma_{y}^{e}$ of 10 .

\begin{tabular}{lllll}
\hline$\sigma_{y}^{e} \mu \mathrm{m}$ & $f \#$ & $\sigma_{\ell}(\mu \mathrm{m})$ & $z_{R}(\mu \mathrm{m})$ & $P_{\ell}(\mathrm{MW})$ \\
\hline 1 & 1 & 0.7 & 8.7 & 7.2 \\
2 & 1 & 0.7 & 8.7 & 20 \\
3 & 1.5 & 1.0 & 19.6 & 30 \\
5 & 1.5 & 1.0 & 19.6 & 104 \\
\hline
\end{tabular}

To lowest order $\sigma_{m}$ is given by: $\sigma_{m}=\sqrt{\left(\sigma_{y}^{e}\right)^{2}+\left(\sigma_{\ell}^{\text {eff }}\right)^{2}}$ (where superscript $e$ refers to electron bunch), but will be larger for high electron beam aspect ratios, where the effects of Rayleigh length become important. The number of Compton photons $N_{C}^{\max }$ produced when electron and photon beams are perfectly aligned is given by [17]

$$
N_{C}^{\max }=\frac{N_{e} P_{\ell} \lambda \sigma_{C}}{\sqrt{2 \pi} h c^{2} \sigma_{m}}
$$

where $\sigma_{C}$ is the Compton cross section and $N_{e}$ is the number of electrons in the bunch. For $\lambda=532 \mathrm{~nm}$ and $N_{e}=0.5 \times 10^{10}$ this becomes

$$
N_{C}^{\max }=1180 \frac{P(\mathrm{MW})}{\sigma_{m}(\mu \mathrm{m})}
$$


A five-point scan of a Gaussian yields a statistical error of $1 \%$ when $N_{C}^{\max } \simeq 2900$ (see [18] for details). Using this as a benchmark and performing full overlap integrals to take into account Rayleigh length effects, allows a laser pulse power to be specified for each electron spot-size as shown in Table 4.1.

If we define relative errors $\delta_{x}=\delta \sigma_{x}^{e} / \sigma_{x}^{e}$ and $r=\sigma_{\ell} / \sigma_{y}^{e}$ then

$$
\delta_{b}^{2}=\delta_{m}^{2}\left(1+r^{2}\right)^{2}+\delta_{\ell}^{2} r^{4}
$$

Assuming we can determine $\sigma_{\ell}$ to $10 \%$ and $\sigma_{b}$ to $1 \%$ (the latter condition sets the minimum laser power in Table 4.1), then in order to avoid being dominated by laser systematics we will require $r<0.3$. So if we achieve $w=2 \sigma_{\ell}=2 \mu \mathrm{m}$ using $f 1.5$ optics, then we need to find a location in the ATF2 line where $\sigma_{b} \simeq 3 \mu \mathrm{m}$. Downstream of the laserwires, at least one dipole is required in order to separate the Compton-scattered photons from the main electron-beam. Thin vacuum windows will be necessary to allow the photons to escape the beam-pipe and experience at the ATF ring shows that additional lead shielding is also important. All these issues place constraints on the possible locations of the laserwires and work has begun to simulate the laserwire operation using a Geant-4 based program [19] and to determine the optimal layout. Possible locations are shown in fig. 4.9.

\subsubsection{ATF Extraction line laserwire}

To start meeting the technological challenges outlined in Sec. 4.3.1, an R\&D project has begun at the ATF extraction line to develop a laserwire using green light focused to a spot-size of $2 \sigma_{\ell}=w \simeq 2 \mu \mathrm{m}$ in a single-shot system. In parallel to the optics design of the ATF laserwire, work is ongoing to explore a fast scanning system based on piezo-driven mirrors (at the PETRA laserwire) and an ultra-fast scanning system based on electro-optic techniques will also be explored. The ATF laserwire system will be installed at the ATF extraction line in the summer of 2005 and data-taking is planned for the end of the year. The results of these tests will determine the optics, DAQ and scanning systems to be used for the ATF2 project. The results of this R\&D project will input directly into determining how well the theoretical values listed in Table 4.1 can be met in practice.

\subsubsection{Timescales}

The ATF extraction line laserwire experiment is currently under construction, aiming at data taking in December 2005. New dedicated laserwire IPs could then be constructed, based on the results from this experiment, early in 2006. Assuming the current ATF extraction line project does not encounter any show-stoppers, the cost of the ATF2 laserwire system, involving possibly several laserwire IPs, could be determined early in 2006 . 


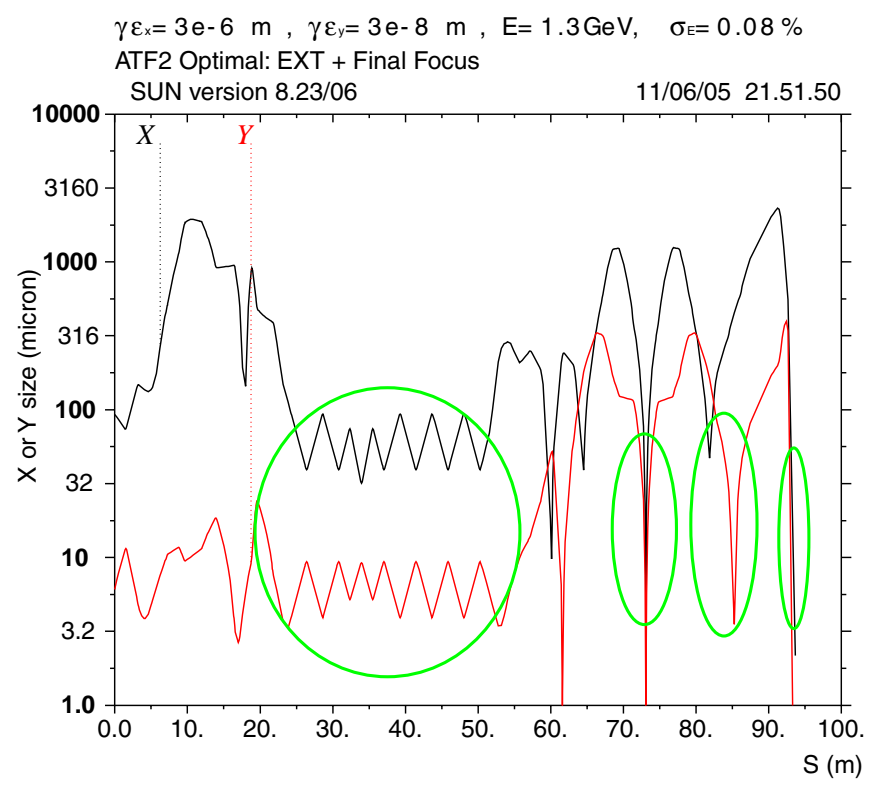

Figure 4.9: Proposed location of laserwire(s).

\subsection{IP beam size monitor}

\subsubsection{Introduction}

This section outlines the proposal to build a laser system to be used with the IP beam size monitor at ATF2. The beam size monitor uses a fringe pattern formed by two interfering laser beams. The fringe pattern transversely overlaps the electron beam. The resulting Compton scattered photons are measured downstream of the interaction point. The modulation depth of the signal is a function of fringe spacing and electron beam spot size and therefore provides the opportunity to measure the electron beam spot size in the horizontal and vertical dimension depending on the arrangement of the fringe pattern. Such a system, also known as a Shintake monitor, was installed in the SLAC FFTB beamline during the 1990s [20]. The system to be installed at ATF2 is intended to measure the transverse electron beam size in the range of $40 \mathrm{~nm}$. The laser system must provide a pulse structure compatible with the ATF2 beam and is intended to simulate ILC IP conditions. The proposal for the new Shintake monitor will be split into two aspects, the laser system and the launch optics.

\subsubsection{Compton scattering for ATF-2 beam conditions}

Table 4.2 summarizes the relevant ATF-2 beam conditions and also compares to the FFTB beam parameters.

The theory of Compton scattering of photons and electrons is well known in the literature [20, 21]. Figure 4.10 shows the Compton scattering cross section as a function of electron energy. Figure 4.11 
Table 4.2: ATF-2 conditions (compared to FFTB conditions)

\begin{tabular}{lll}
\hline Parameter & FFTB & ATF-2 \\
\hline e- beam energy & $46.6 \mathrm{GeV}$ & $1.3 \mathrm{GeV}$ \\
Bunch charge & $1.6 \mathrm{nC}$ & $1.6-3.2 \mathrm{nC}$ \\
Electrons per bunch & $1 \times 10^{10}$ & $1-2 \times 10^{10}$ \\
Bunch length & $\sim 1 \mathrm{~mm}(\sim 3 \mathrm{ps})$ & $\sim 5 \mathrm{~mm}(\sim 16 \mathrm{ps})$ \\
Beam size at IP & $\sigma_{x}=1.7 \mu \mathrm{m} \sigma_{y}=60 \mathrm{~nm}$ & $\sigma_{x}=3 \mu \mathrm{m} \sigma_{y}=40 \mathrm{~nm}$ \\
\hline
\end{tabular}

is a graph of Compton cross section as a function of laser wavelength calculated for the ATF-2 beam energy of $1.3 \mathrm{GeV}$. Although the Compton cross section at different laser wavelength is similar at electron energies of $1.3 \mathrm{GeV}$ we propose to use the second harmonic frequency of a $\mathrm{Nd}$ doped laser (Nd:YAG or Nd:YLF) laser to produce a wavelength of $532 \mathrm{~nm}$ or $527 \mathrm{~nm}$ to obtain the fringe spacing necessary for a $40 \mathrm{~nm}$ spot size measurement. The fringe spacing is a function of wavelength and laser beam crossing angle $(d=\lambda /[2 \sin (\phi / 2)])$. The launch optics will provide several crossing angles to provide the means to measure a range of beam sizes. The angles of the FFTB design [20, 21] are 6, 30 and 174 degrees. The spatial frequency of the fringe pattern that presents the target to the electrons results in a modulation of the Compton scattered photons. The modulation depth of the measured Compton scattered photon signal will be higher as the ratio of electron spot size and fringe spacing decreases. The measurable spot size can be estimated by the following formula:

$$
\sigma_{s}=\frac{s(\phi)}{2 \pi} \sqrt{2 \ln \left|\frac{\cos (\phi)}{M}\right|}
$$

With $\sigma_{s}=$ electron beam spot size, $\phi=$ laser beam crossing angle, $M=$ modulation depth.

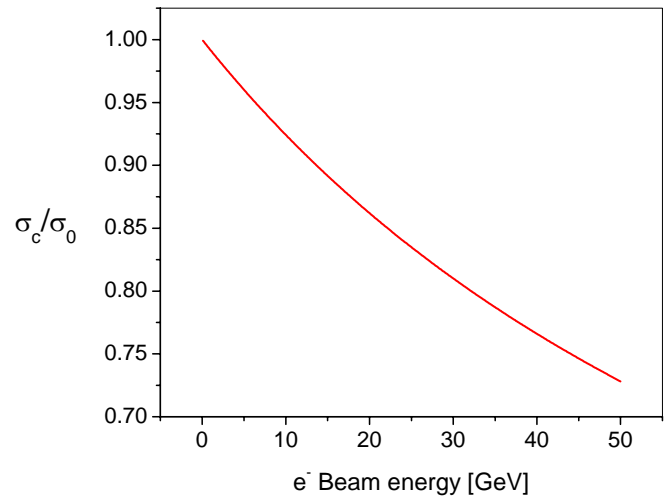

Figure 4.10: Ratio of Compton and Thompson cross section as a function of beam energy.

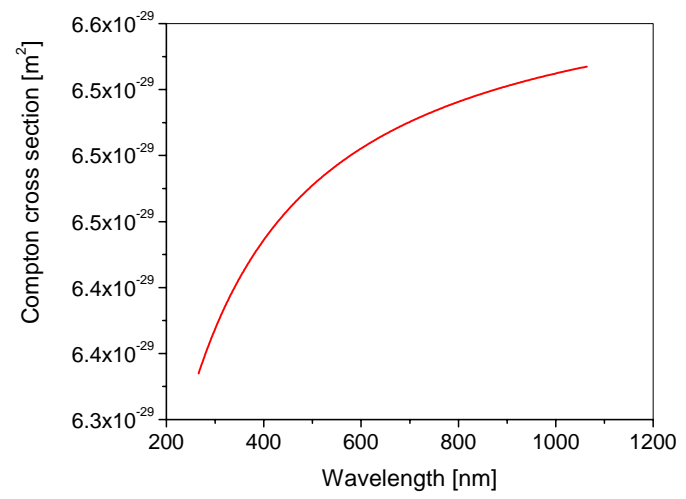

Figure 4.11: Compton cross section as a function of laser wavelength.

Table 4.3 summarizes the minimum measurable spotsize using $532 \mathrm{~nm}$ photons. 
Table 4.3: Minimum measurable spotsize using $532 \mathrm{~nm}$ photons for modulation depths $10 \%$ and $90 \%$.

\begin{tabular}{lll}
\hline Crossing angle & Spot size at $\mathrm{M}=0.1$ & Spot size at $\mathrm{M}=0.9$ \\
\hline $174^{\circ}$ & $91 \mathrm{~nm}$ & $19 \mathrm{~nm}$ \\
$30^{\circ}$ & $340 \mathrm{~nm}$ & $45 \mathrm{~nm}$ \\
$6^{\circ}$ & $1.7 \mu \mathrm{m}$ & $362 \mathrm{~nm}$ \\
\hline
\end{tabular}

In order to obtain a reasonable signal to noise ratio, it is important to have adequate laser power. The number of scattered x-ray photons $\left(n_{\gamma}\right)$ can be calculated using:

$$
n_{\gamma}=\sigma_{c} \times n_{p} \times D \times n_{e}
$$

with $\sigma_{c}=$ Compton cross section, $n_{p}=$ photons per $\mathrm{cm}^{3}, D=$ diameter of laser spot and $n_{e}=$ number of electrons. Using a $10 \mathrm{MW}$ laser we obtain a $100 \mu \mathrm{J}$ pulse energy in a $10 \mathrm{ps}$ long pulse. If the light is focused to a spot diameter of $100 \mu \mathrm{m}$, the number of generated x-ray photons is $>700$.

\subsubsection{Laser system}

A laser system that meets the requirements for an ATF-2 beam size monitor has been developed at the DESY TTF facility [22]. A conceptual diagram is depicted in Figure 4.12. This laser system generates the fourth harmonic of the fundamental wavelength of a Nd:YLF laser system. We propose to use a simplified version as the ATF-2 IP beam size monitor. The system consists of a mode locked oscillator that is synchronized to the machine master clock. The single pulses have a length of $\sim 12$ ps. An electro-optical modulator is used to reduce the repetition rate of the oscillator to match the single bunch spacing of the e- beam. Using a Pockel cell, the bunch pattern is chopped to match the 1 , 5 or $10 \mathrm{~Hz}$ repetition rate of the bunch train. The pulse train is pre-amplified by a chain of two diode pumped single pass amplifiers and brought to the final pulse energy by two flash-lamp pumped amplification stages. The single pulse energy of this system (in the IR) reaches $5.9 \mu \mathrm{J}$ at $1 \mathrm{MHz}$ and $3.2 \mu \mathrm{J}$ at $3 \mathrm{MHz}$ repetition rate. The flash-lamp pumped stages boost the pulse energy up to $\sim 300 \mu \mathrm{J}$ at $1 \mathrm{MHz}$ and $\sim 140 \mu \mathrm{J}$ at $3 \mathrm{MHz}$. Frequency conversion takes place after amplification.

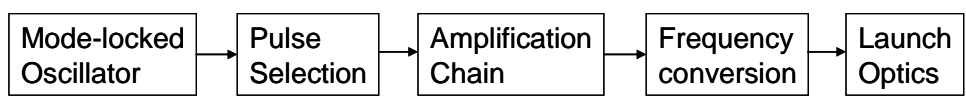

Figure 4.12: Conceptual diagram of laser system components.

For an ATF-2 IP beam size monitor system only one flash-lamp pumped amplifier would be necessary and only one frequency conversion step would be necessary to achieve the required laser pulse energy and wavelength requirements. As the experience with the FFTB beam size monitor has shown [23, 24], a significant R\&D effort is necessary to achieve optimal operating conditions such as beam quality, stability and vibration isolation. 


\subsubsection{Laser system alternative}

A much simpler laser system could be used if only the spot size measurement of a single bunch is required. Low cost diode pumped, frequency doubled Nd:YAG or Nd:YLF systems are readily available. Pulse lengths of a Nd:YAG and Nd:YLF system are typically $<10$ ns and $100 \mathrm{ns,} \mathrm{respectively.}$ Such a design would lead to a partial overlap of laser and electron pulse. The major disadvantage is that only one pulse can be sampled in a pulse train.

\subsubsection{Launch Optics system}

The mechanical components of the optical system that split and recombine the laser beam and provide transport to the interaction point are available from the FFTB beam size monitor. A simplified schematic is shown in Figure 4.13. The system is mounted on an optical table and can be shipped to the ATF-2 facility. The optical components (beamsplitters, mirrors, lenses, etc.) must be replaced with optics that have the appropriate coatings to match the wavelength of the laser.

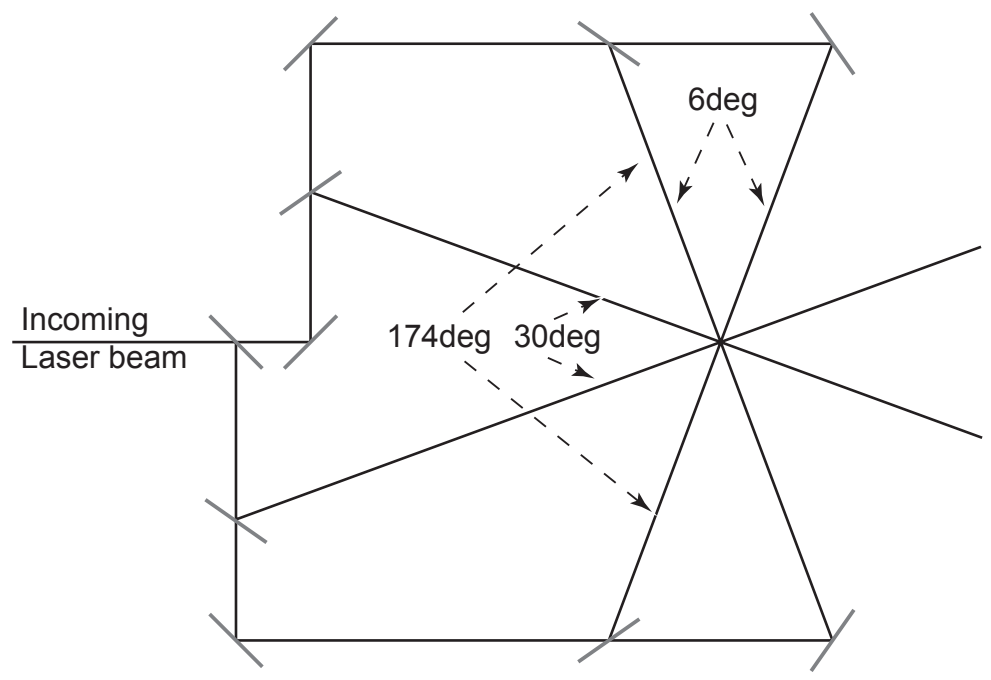

Figure 4.13: Scheme of launch system optical layout showing crossing angles of $6^{\circ}, 30^{\circ}$ and $174^{\circ}$.

\subsubsection{Overlap with polarized source development}

The laser system will have similar characteristics to a laser system suitable to operate a polarized source with an ILC bunch structure. We expect some overlap of polarized source laser and Shintake monitor laser system development. However, the fundamental difference between the laser systems is the required pulse length. This may lead to two different laser systems. The polarized source laser requires a single pulse length of several hundreds of picoseconds to a nanosecond. This long pulse length is necessary to overcome the space charge effects associated with current extraction from the 
DC gun of the polarized source. The wavelength of a source laser is $\sim 800 \mathrm{~nm}$. The long pulse length makes the source laser system more complex. 


\section{ATF extraction line \& extraction line diagnostics}

\subsection{Emittance and orbit jitters in the extraction line}

\subsubsection{Vertical Emittance}

As shown in Fig. 5.1, the vertical emittance in the extraction line, measured by conventional wire scanners, has been larger than the emittance in the damping ring, measured by the laserwire monitor $[2,25]$.

The observed intensity dependence of the vertical emittance is also much stronger in the extraction line than in the damping ring. This dependence is also larger than the intensity dependence of the longitudinal and horizontal emittances. This strong intensity dependence cannot be explained by linear coupling between the vertical and either of the other two axes. We suspect that unknown nonlinear fields in the extraction kicker and the septum magnets cause higher-order $\mathrm{x}-\mathrm{y}$ and/or energy-y coupling. The normalized vertical emittance (at $N=5 \times 10^{9}$ ) is about $48 \mathrm{~nm}$, which is larger than in the damping ring (about $15 \mathrm{~nm}$ ) by a factor of three, while the nominal ATF2 goal emittance is $30 \mathrm{~nm}$. If the vertical emittance cannot be reduced to the nominal value, it would make the vertical beam spot size larger than nominal size by about $30 \%$, for an intensity of $5 \times 10^{9}$. For $2 \times 10^{10}$ the blow up would be even larger.

\subsubsection{Orbit jitter}

Significant transverse orbit jitter as well as drifts are caused by energy changes in the damping ring through residual dispersion in the diagnostics region of the extraction line, where the design dispersion is zero. Both linear and second order dispersion are important in this case. Energy oscillations, or synchrotron oscillations, can be randomly excited, probably by noise in the low level RF system and, in multi-bunch operation, also by a longitudinal coupled-bunch instability. The jitter of the beam energy due to these oscillatione is between about $1 \times 10^{-4}$ to $2 \times 10^{-4}$ (rms) in single bunch operation without feedbacks. An RF feedback has been implemented to reduce the energy instability to about $7 \times 10^{-5}[26,27]$. In addition, a slow momentum drift in the ring has been observed. The effect of the energy jitter on the extraction orbit obviously depends on how well the extraction line has been tuned, especially, on the magnitude of the residual dispersion. However, if the dispersion is matched in the extraction line, the jitter downstream should be small, even if the dispersion is not zero at the extraction point in the ring. What is most important is that the dispersion in the ring does not vary with time.

The horizontal orbit jitter in the extraction line was studied in detail when investigating the double kicker performance [28]. The jitter was found to be less than 20 microns at five wire scanners, which is 


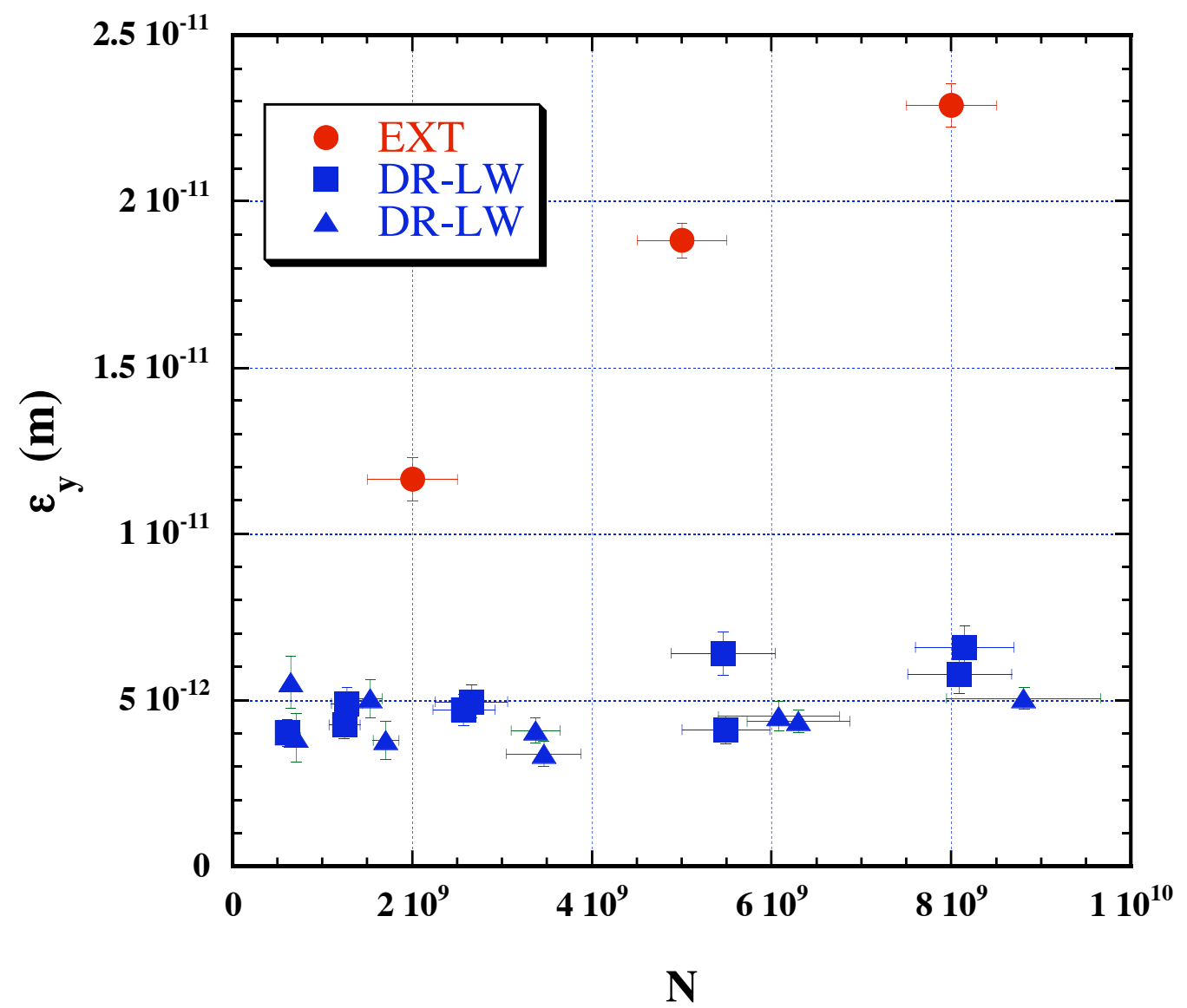

Figure 5.1: Vertical emittance vs. bunch intensity $N$, measured in the extraction line using wire scanners (EXT) and measured in the damping ring using the laserwire monitor (DR-LW).

about $20 \%$ of the beam size. Vertical orbit jitter was recently measured using a cavity BPM system. The magnitude of the jitter seems to change day by day, probably due to different dispersion correction conditions. Under good conditions, the vertical position jitter was less than $2 \mu \mathrm{m}$ [29]. The calculated beta-function is about $2.0 \mathrm{~m}$ at the location of the measurement, so that the $2 \mu \mathrm{m}$ corresponds to about $40 \%$ of the nominal beam size (for a nominal normalized vertical emittance $30 \mathrm{~nm}$ ). Another jitter estimate was provided by the US nano-BPM study, namely 20 micron horizontally and 3.5 micron vertically [27] at a location with model beta functions $\beta_{x}$ equal to about $2 \mathrm{~m}$ and $\beta_{y}$ about $3 \mathrm{~m}$. The vertical orbit jitter has been also estimated from wire scanner data, as from $30 \%$ to $40 \%$ of the nominal beam size. From these numbers, the horizontal jitter is about $20 \%$, and the vertical jitter about $40 \%$ of the nominal beam size (for a nominal normalized horizontal emittance of $30 \mathrm{~nm}$ ). 


\subsubsection{Plan for improving the beam quality}

For the ATF-2 design goal, it will be necessary to reduce the vertical emittance to the nominal value of $30 \mathrm{~nm}$ normalized, which corresponds to twice the emittance measured by the laserwire scanner in the damping ring. Stabilization of the orbit, i.e., reducing the jitter from about $40 \%$ of the beam size to $5 \%$, will also be required.

The jitter and emittance will be reduced by correcting the vertical dispersion, second order dispersion, and coupling in the extraction line, as described in Section 5.2.

At the same time, effort will be made to reduce the sources of instability and drift:

- One prominent source of drift is changes in the outside temperature, which affects the cooling water flow, causing vibration and drift. A possible solution for limiting the effects of temperature variation is to limit the operation period for ATF/ATF-2 from November to April, keeping a total of 22 weeks of operation per year. This is still under discussion. Another, more expensive solution would be to upgrade the cooling water system, by the addition of new controls and by modifying the cooling water flow, so as to render it less sensitive to the outside temperature.

- Improvements and fine tuning of the double kicker system are necessary to deliver a stable flat beam.

- A feed-forward system for control of beam position and angle between the damping ring and the extraction line will help stabilize the beam trajectory in ATF-2.

- Drifts of the optics parameters need to be quantified and better understood, both in the ring and in the extraction line.

- The origin of $x-y$ cross coupling and energy-y coupling in the extraction line will further be investigated. Independently, these aberrations will be measured and corrected as described in Section 5.2.

- If the emittance growth occurs in the extraction line, as presently thought, wakefields at the kicker or septum are a possible cause. This aspect also demands further scrutiny.

- We are exchanging the extraction kicker system this year with one that has a better understood skew field component. The expected steering and positioning tolerances of the magnet $( \pm 100 \mu \mathrm{m}$ vertical offset) should be achievable.

- We expect to upgrade the beam position monitor system in the ring to provide about a factor 50 improvement in resolution. This should allow a better correction of coupling and dispersion. The present system has about $4 \mu \mathrm{m}$ resolution and $100 \mu \mathrm{m}$ rms offsets. Using high speed averaging and higher dynamic range ADC's with an automatic calibration system we hope to achieve about $100 \mathrm{~nm}$ resolution. Experience has shown that the emittance tuning greatly benefits from improved BPM performance. 
- The observed changes in the extraction line spurious dispersion result from trajectory drifts due to kicker drifts (and other power supply instabilities). This may be improved by the new kicker system.

\subsubsection{Summary}

The present performance of the extracted beam is summarized as follows:

- The vertical emittance is a factor 1.6 larger than nominal (at $N=5 \times 10^{9}$ ), and it strongly depends on the intensity.

- The vertical orbit jitter is about $40 \%$ of the nominal beam size.

- This performance is good enough to start the ATF-2 experiment.

- A plan to improve the beam quality with multiple approaches has been developed.

\subsection{Vertical dispersion, 2nd order dispersion, and coupling correction in extraction line}

The operational experience to date, and the emittances and beam stability achieved in the existing ATF extraction line (EXT) are described in the previous section. In particular, the vertical emittance measurements in the DR (by laserwire) and in EXT (by wire scanner), shown in Fig. 5.2, demonstrate that the vertical emittance dilution between the DR and the EXT wire scanners even under the best conditions has been $200 \%$ for single-bunch low intensity, and $300 \%$ for single bunch ATF2 intensity $\left(N \approx 5 \times 10^{9}\right)$. The layout of the existing ATF extraction line is shown in Fig. 5.2; the optics of the EXT diagnostic section is shown in Fig. 5.3.

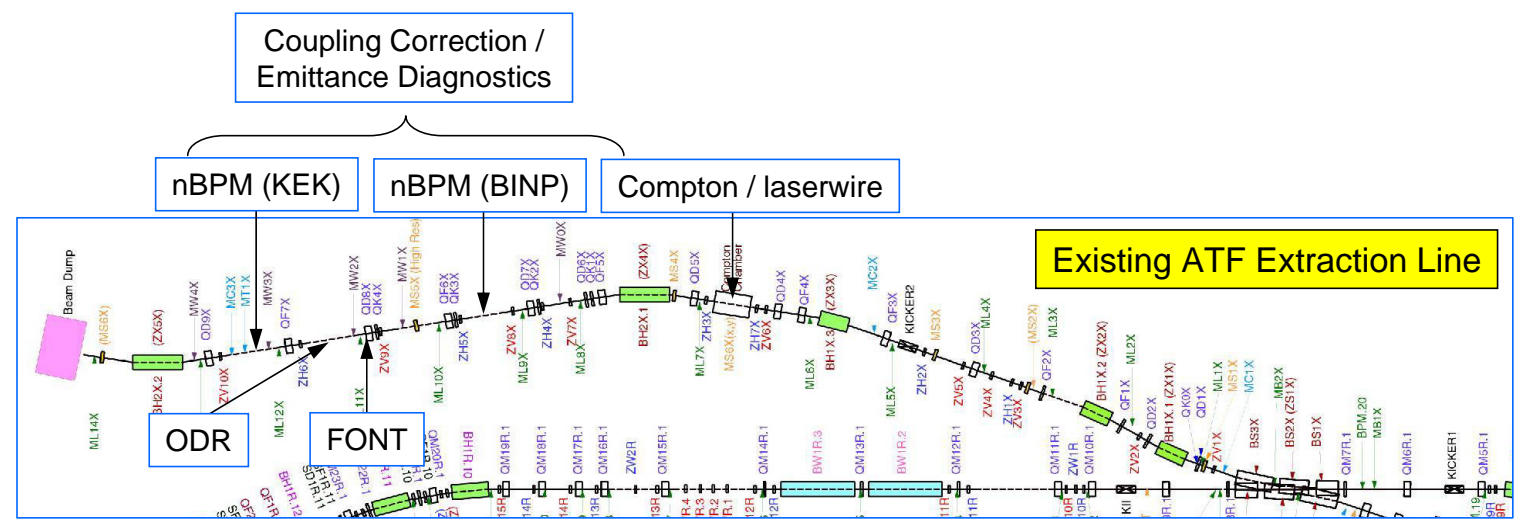

Figure 5.2: Layout of existing EXT line showing locations of various R\&D experiments. 


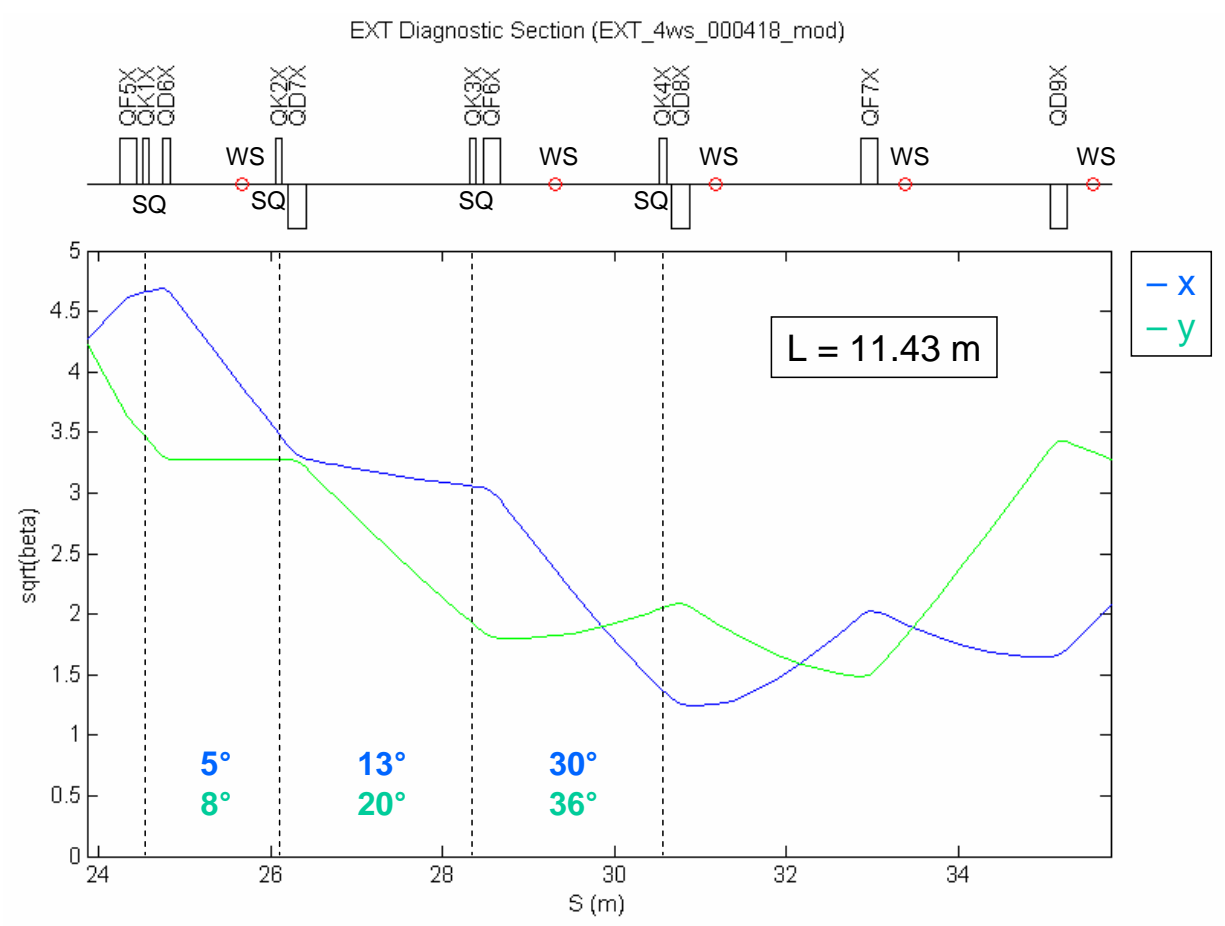

Figure 5.3: Existing ATF EXT diagnostic section showing skew quads (SQ), wire scanners (WS), and betatron phases.

It is clear that accurate dispersion and coupling correction in EXT are essential and directly impact ATF2 goals A and B. The relevant experience with correction of vertical dispersion, linear and 2nd order horizontal dispersion, and transverse coupling can be summarized as follows:

1. Linear horizontal dispersion correction is performed routinely using 2 normal quadrupoles (QF3X and QF4X) which have been selected because they strongly influence the residual dispersion in the diagnostic section but introduce minimal beta mismatch over the normal range of corrections.

2. Vertical dispersion correction is also in good shape now using 2 EXT skew quadrupoles (QS1X and QS2X) as suggested by Paul Emma [30].

3. Correction of 2 nd order horizontal dispersion is now being tested using 3 newly installed FFTB sextupoles provided by SLAC (explained further below).

4. Coupling correction has been problematic. Although there are 4 skew quadrupoles (QK1XQK4X) installed for coupling correction in the EXT diagnostic section, the present nominal EXT optics, shown in Fig. 5.3, is not optimal for this correction and some phases of coupling cannot be corrected. This has been observed in several failed attempts to reduce the measured vertical emittance at the EXT wire scanners by adjusting the strengths of the skew quadrupoles. 


\subsubsection{Measurement and correction of $2 \mathrm{nd}$ order dispersion}

The dispersion measurement procedure consists of changing the beam energy in the DR (by changing the RF frequency) and measuring the change in beam position at the EXT BPMs. Results of a typical measurement of 1st and 2nd order horizontal dispersion in EXT are shown in Fig. 5.4. Measurements of the 2nd order dispersion at BPMs upstream of the diagnostic section have large errors because off-energy beam motion is dominated by the large design linear horizontal dispersion there. This is also true for measurements of vertical dispersion. Measurements in the diagnostic section, where the linear horizontal dispersion is nominally zero, can be performed with better accuracy.
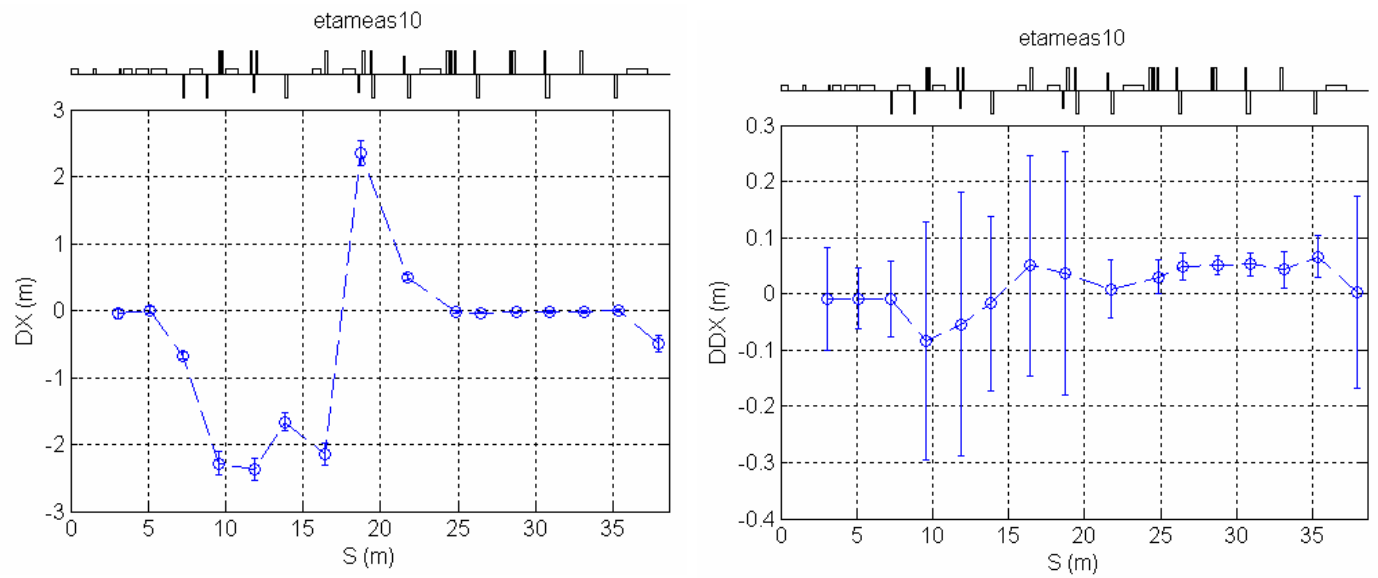

Figure 5.4: Results of a typical measurement of 1st and 2nd order horizontal dispersion in EXT.
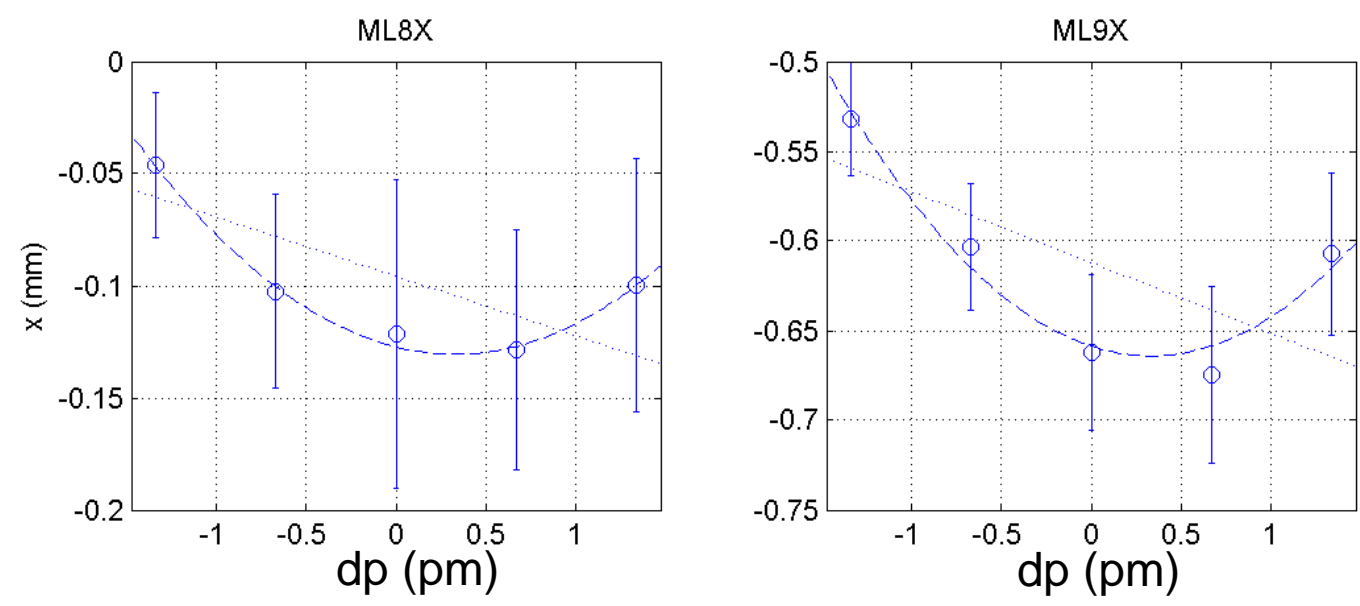

Figure 5.5: Variation of horizontal beam position with energy offset at 2 diagnostic section BPMs showing quadratic dependence. This illustrates the procedure for measuring 2 nd order dispersion and typical measurement errors.

The main factors that limit the resolution of EXT dispersion measurements are the large design linear 
dispersion (about $2.5 \mathrm{~m}$ ), which limits the allowable beam energy change to about $\pm 0.65 \%$, and other uncontrolled energy changes (both jitter and drift) in the DR. Other factors include BPM resolution and transverse beam jitter. Modification of the EXT optics to reduce the linear dispersion could be considered, but this must be weighed against the fact that the large dispersion is desirable for precise longitudinal phase space (energy spread) diagnostics, and that any modification may need to satisfy additional constraints imposed by the double kicker jitter reduction scheme.
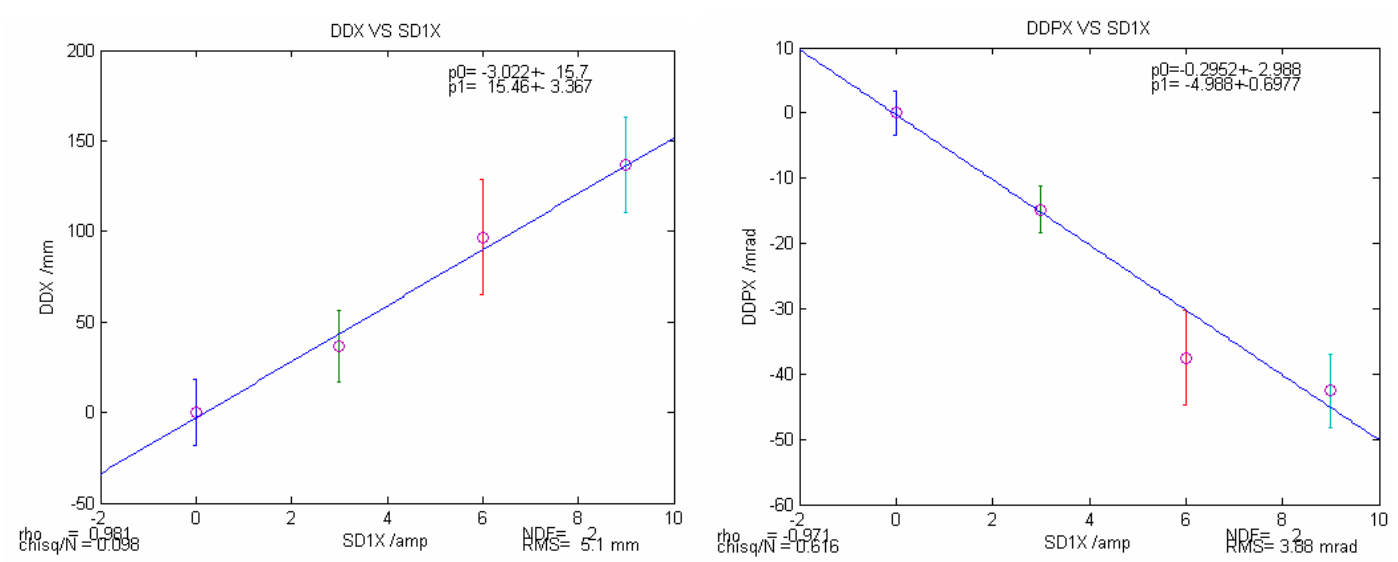

Figure 5.6: Measured second order dispersion and its derivative (along z) versus strength of sextupole SD1X.

Three sextupoles were installed in EXT for correction of the 2nd order horizontal dispersion (actually only two are required; a third was added to allow simultaneous minimization of the vertical chromaticity). Preliminary measurements have shown that these sextupoles affect the 2nd order horizontal dispersion and its derivative as expected (see Fig. 5.6), and linear combinations of the sextupole strengths can be used to make corrections.

\subsubsection{Design of an expanded diagnostics section}

In order to achieve the ATF2 goals, we have designed an expanded diagnostics section for EXT.

It is relevant to mention studies of the skew correction and emittance diagnostics section designed for the NLC. Studies have shown that measuring the full 4D phase space of the beam, even under optimal conditions, is so sensitive to beam size measurement errors as to be essentially useless [31]. An "ideal" system consists of a compact orthogonal coupling correction section followed by a 4-wire-scanner 2D (projected) emittance measurement section. This system would be robust even in the presence of measurement errors. In practice, coupling correction will consist of sequentially minimizing the projected vertical emittance with respect to each of the 4 skew quadrupoles. The $500 \mathrm{GeV}$ system designed for NLC is shown in Fig. 5.7 (top plot).

When redesigning the EXT diagnostic section, it is also important to take into account different R\&D experiments which use this section (nBPM SLAC/KEK, ODR, FONT, Compton/laserwire), as shown 
in Fig. 5.2. Therefore, if possible, the redesigned diagnostic section should also have sufficiently long drifts that could be suitable for today's and for future instrumentation.

A suggested design (EXT2) for the optimal skew correction / emittance measurement section for ATF-2 follows the NLC design and is shown in Fig. 5.7 (bottom plot). The betatron phase advances are very close to ideal, and there are several sufficiently long drifts where R\&D of new diagnostics could be performed. The matching to the new diagnostics section required the addition of a new quadrupole inserted in the existing beamline between QF3X and BH1X.3 as shown in Fig. 5.8. 

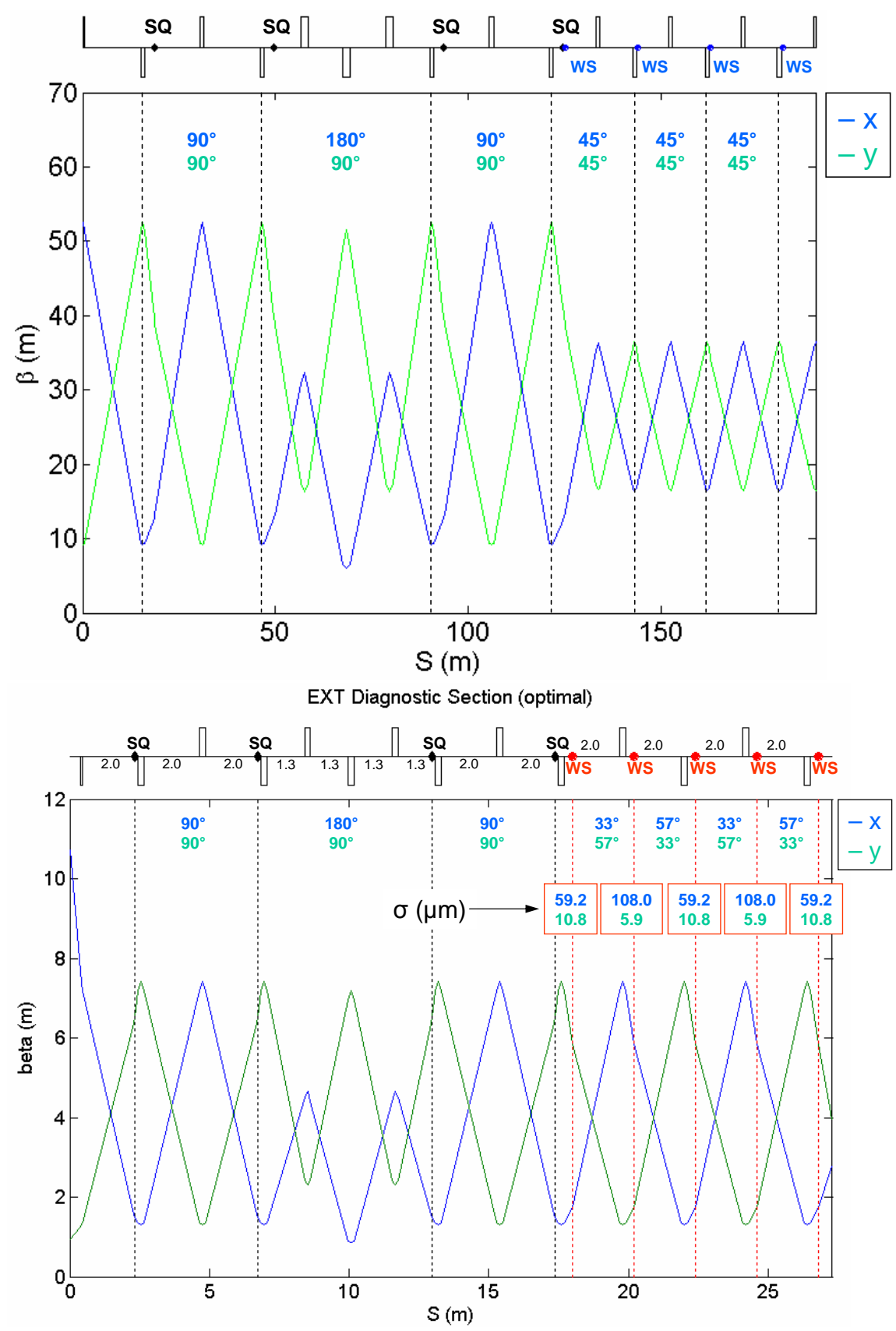

Figure 5.7: Ideal skew correction / emittance measurement section for NLC (top plot) and the new ATF2 skew correction / emittance measurement section (bottom plot). 


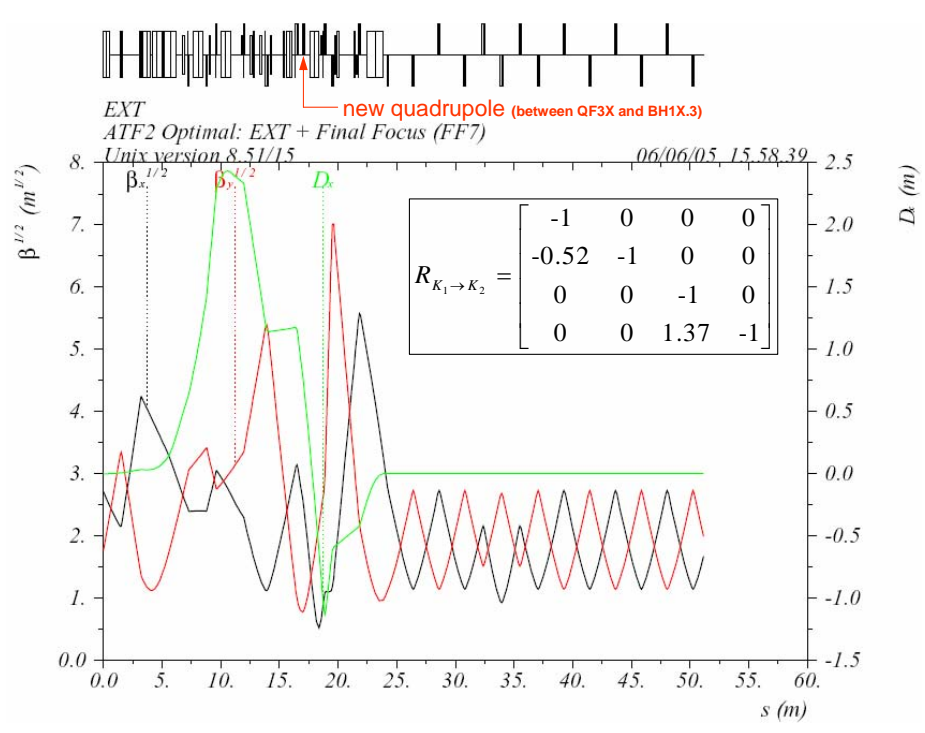

Figure 5.8: ATF2 extraction line (EXT2) with expanded skew correction / emittance measurement section showing location of an additional quad. The partial -I transfer matrix between kickers for this optics is also shown.

\subsubsection{Simulation of beam correction with new diagnostics}

We have performed simulations of emittance correction in the extraction line using the redesigned diagnostics section. The assumed parameters are the following: perfect beams from the Damping Ring $\left(\varepsilon_{x}=2 \times 10^{-9} \mathrm{~m}, \gamma \varepsilon_{y}=3 \times 10^{-8} \mathrm{~m}\right)$; perfect Final Focus (chicane to IP); vertical dipole misalignments (some EXT dipoles are assumed to have nonzero sextupole components) of $100 \mu \mathrm{m}$ (rms); horizontal quadrupole misalignments of $50 \mu \mathrm{m}$ ( $\mathrm{rms})$; vertical quadrupole misalignments of $30 \mu \mathrm{m}$ ( $\mathrm{rms}$ ); quadrupole rolls of $0.3 \mathrm{mrad}(\mathrm{rms})$; BPM resolution of $5 \mu \mathrm{m}$ ( $\mathrm{rms})$; wire scanner rolls: $-0.2^{\circ} \leq \theta \leq+0.2^{\circ}$ (uniform); wire scanner beam size errors: $\sigma=\sigma_{0}\left(1+\Delta \sigma_{\text {relative }}\right)+\Delta \sigma_{\text {absolute }}$ (the values for beam size errors will be discussed below). Effects which were not included in the simulations are: quadrupole strength errors $(\Delta K / K)$, BPM offsets, and BPM rolls.

The simulated vertical emittance correction procedure is as follows:

1. apply errors

2. steer flat (EXT2 only)

- each diagnostic section quadrupole has an associated BPM

- each diagnostic section QF has an associated XCOR

- each diagnostic section QD has an associated YCOR

3. launch into FF

- use 2 virtual correctors 

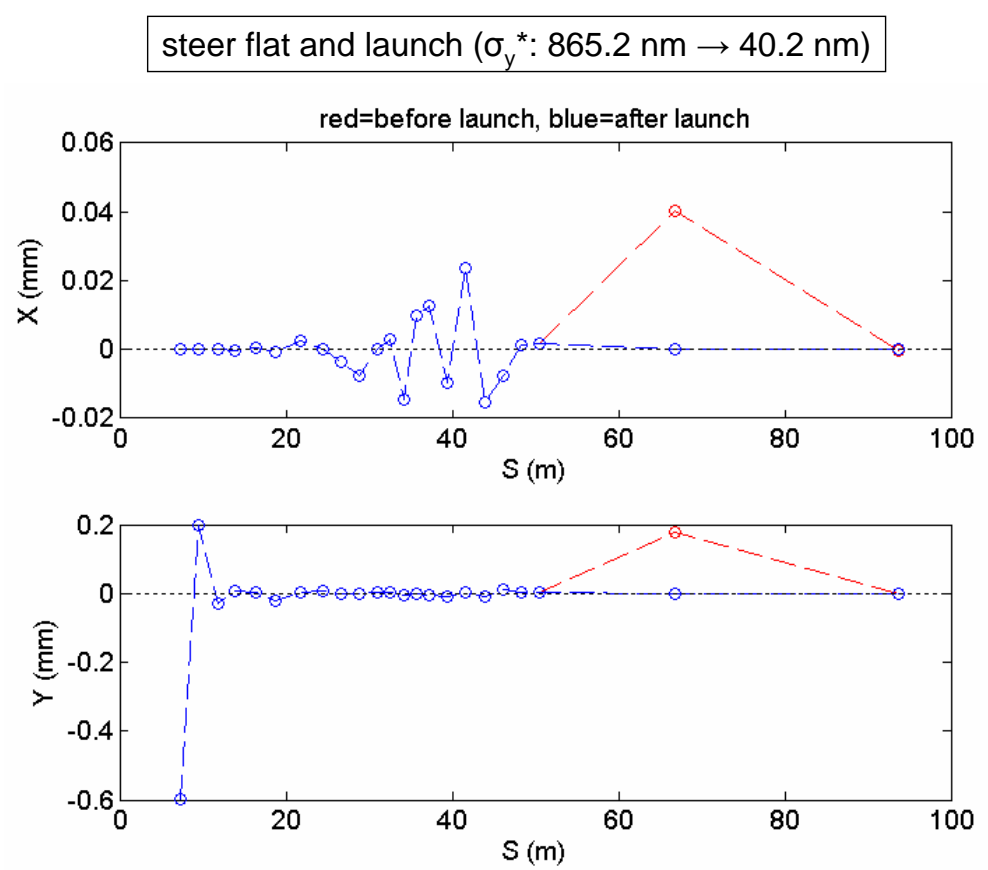

Figure 5.9: Simulation of correction in EXT2, beam orbit before and after steer/launch correction.

- steer to 2 virtual BPMs (one at the IP and one $90^{\circ}$ upstream)

- virtual BPMs are perfect

4. measure dispersion in diagnostic section

- scan input beam energy

- measure orbits

- fit position vs energy at each BPM

5. correct vertical dispersion in diagnostic section

- back propagate measured $\eta_{y}$ to start of diagnostic section to get $\eta_{y 0}$ and $\eta_{y 0}^{\prime}$

- correct using skew quads (QS1X and QS2X) in dispersive region of EXT2

6. correct coupling

- scan 4 skew quadrupoles sequentially

- deduce projected $\varepsilon_{y}$ from wire scanner measurements

- set each skew quad to minimize projected $\varepsilon_{y}$.

An example of the simulated beam trajectory before and after the steer/launch procedure is shown in Fig. 5.9. The vertical beam size at the IP, for different simulation seeds, after each step of the correction procedure is shown in Fig. 5.10. For the values of errors chosen the nominal beam size is already achieved after the dispersion correction step. This is not consistent with measurements in the existing beamline where the same procedures have been unable to reduce the vertical emittance dilution to less than $300 \%$ for a single bunch of ATF-2 intensity. Clearly more errors must be 

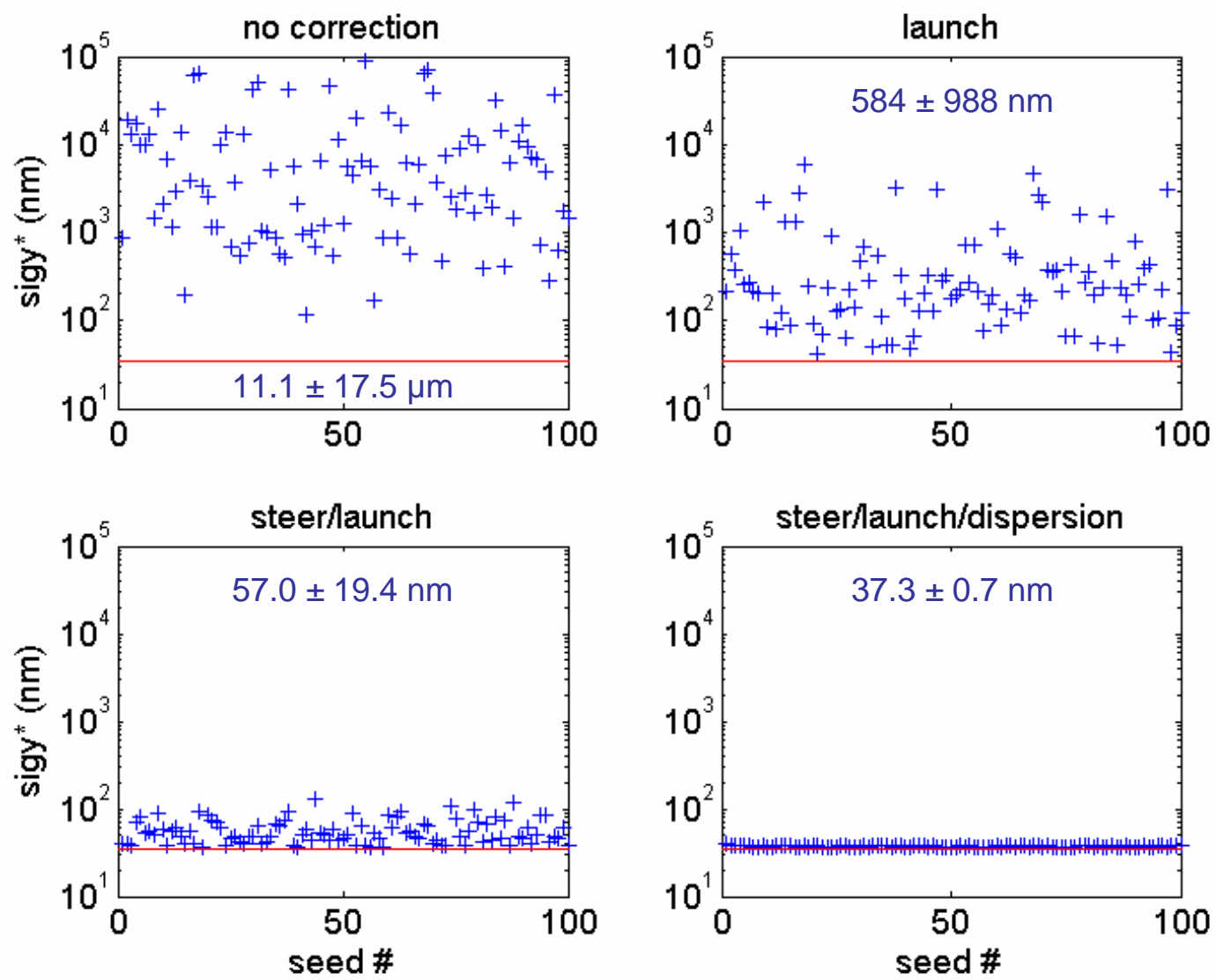

Figure 5.10: Results of simulated corrections in EXT2, vertical IP spot size versus seed number at various stages of the procedure.

included in future simulations, such as large anomalous skew quadrupole fields in the DR extraction kicker and septa which, in simulations, produce the observed vertical emittance dilution after steering and dispersion correction. Coupling correction, with both perfect wire scanners and with beam size measurement errors, will then be simulated in order to assess the system performance. Simulations of the tolerances on the correction of residual dispersion and coupling imposed by goals $\mathbf{A}$ and $\mathbf{B}$ are also being performed. (The latter should be determined by the range of the dispersion correction knobs at the IP.) 


\section{Kicker}

This section describes the design considerations for the stipline kicker which would extract an ILC-like train from ATF. Two possibilities are presently being considered. The first, which is considered as a baseline, uses two sets of kickers, distributed in the ATF ring, as described in Section 6.1. An idea of a low aperture stripline kicker is also described in Section B.

\subsection{Kicker design, to produce the ILC-like train}

The purpose of this project is to develop a prototype ILC damping ring kicker system, with a 5 to 10 nanosecond full width pulse, for extracting single bunches from the ATF ring. The prototype will serve in 2 ways, first to provide experience working with kicker technology similar to that which will be used in the ILC and second to provide a short, low emittance bunch train with characteristics very similar to that of the ILC. As explained below, it is possible to provide about 30 bunches with inter-bunch spacing near that of the ILC.

The magnet parameters for the proposed ATF ring extraction system are listed in Table 6.1. The table also lists the TESLA TDR damping ring and existing ATF kicker parameters for comparison.

Table 6.1: Kicker parameter comparison.

\begin{tabular}{llll}
\hline & $\begin{array}{l}\text { Fast extraction } \\
\text { from ATF }\end{array}$ & Existing ATF & $\begin{array}{l}\text { TESLA TDR } \\
\text { damping ring }\end{array}$ \\
\hline Type & Stripline & $\begin{array}{l}\text { Ferrite loaded } \\
\text { transmission line }\end{array}$ & Stripline \\
Kick angle & $5.0 \mathrm{mrad}$ & $5.0 \mathrm{mrad}$ & $0.6 \mathrm{mrad}$ \\
Kicker integrated strength & $220 \mathrm{Gauss}-\mathrm{m}$ & $220 \mathrm{Gauss}-\mathrm{m}$ & $100 \mathrm{Gauss}-\mathrm{m}$ \\
Magnet length & $2.2 \mathrm{~m}$ & $0.4 \mathrm{~m}$ & $10 \mathrm{~m}$ \\
Diameter & $12 \mathrm{~mm}$ & $16 \mathrm{~mm}$ & $60 \mathrm{~mm}$ \\
Peak Voltage (@50 ohms) & $20 \mathrm{KV}$ & $40 \mathrm{KV}$ & $10 \mathrm{KV}$ \\
Pulser type & FET/shock line & Thyratron & FET \\
\hline
\end{tabular}

Only one magnet technology appears appropriate, an in-vacuum stripline pair. This device is free of ceramic or ferrite and must be fed from the downstream end, so that the pulse travels in the direction opposite to the beam motion, in order to have a good kick amplitude. When properly designed, the total kicker pulse width is twice the propagation delay of the stripline [32].

There is little room within the existing ATF lattice for an additional $2.2 \mathrm{~m}$ of kicker striplines. A workable initial design has been found that uses two sets of striplines, one $0.8 \mathrm{~m}$ long at the end of the south straight section near ZH39R with a field of $50 \mathrm{G}$ and the other $1.4 \mathrm{~m}$ long in the region that includes the existing ferrite loaded kicker. The latter must have $100 \mathrm{G}$ field. Thus, on its last 


\section{Timing Scan(FPG5-1PM)}

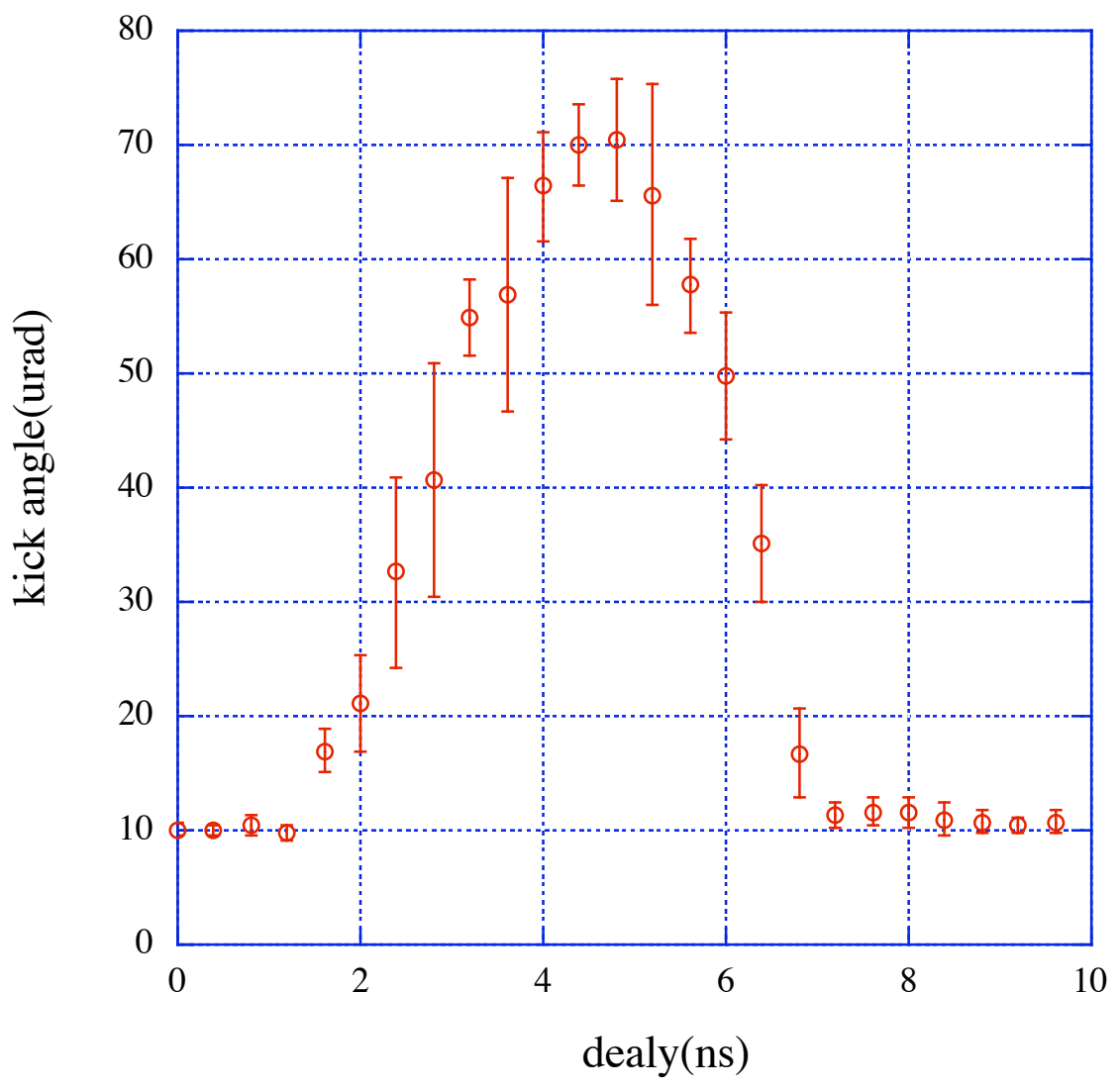

Figure 6.1: Results of tests of MOS-FET power transistor-based pulser at ATF.

transit through the east arc of the ring, the beam trajectory deviates from the nominal with oscillation amplitude of $\pm 3 \mathrm{~mm}$. The effect this oscillation has on the emittance of the beam must be modeled. The focus magnet QM6R.1 will be moved downstream and its strength increased. This change must be properly matched with the ring lattice.

Several high current pulser technologies are under consideration. A ferrite loaded shock-line must be used to decrease the rise time of the pulse. Several tests will be done using a MOS-FET power transistor-based pulser. These devices have been tested at ATF, TTF and DARHT [32, 33]. Fig. 6.1 shows the result of one such test at ATF. The figure shows the response of the stored beam to a kick from a fast rise-time solid state pulser. The horizontal scale is the kicker trigger delay in nanoseconds, with the risetime on the right hand side of the pulse. The rise time is $2 \mathrm{~ns}$ and the full width of the pulse is $5.5 \mathrm{~ns}$.

The nominal ATF bunch spacing, $2.8 \mathrm{~ns}$, with one bunch for every 2 RF buckets, would be increased by at least a factor of 2 by modifying the injector photocathode gun laser. This roughly matches the 
expected kicker pulse duration of $5 \mathrm{~ns}$. The number of bunches with that minimum spacing that can be injected into ATF is roughly 30.

Since the ATF harmonic number is 330, and the kicker pulse must steadily advance to the next bunch in a given train during the extraction sequence, we can, for example, choose a kicker inter pulse delay of 222 RF buckets. (Alternatively, the pulse could move to the previous bunch.) Thus 3 bunches are extracted every two turns and the appropriate inter-bunch spacing in the ring is 6 buckets. The maximum train length with the present injection kicker system is about 60 ring RF buckets, making it possible to have 3 trains of 10 bunches each spaced by 56 ring RF buckets. Table 6.2 lists the parameters of this model. It should be emphasized that the model is devised to provide an evenly spaced sequence of bunches in the extraction line with close to ILC spacing. Other inter bunch spacing intervals can be arranged for kicker tests etc.

Table 6.2: Ring and extracted bunch timing.

\begin{tabular}{ll}
\hline \hline Ring harmonic number & 330 \\
Rotation frequency & $2.164 \mathrm{MHz}$ \\
Number of trains & 3 \\
Train frequency & $6.491 \mathrm{MHz}$ \\
Inter bunch spacing & $8.4 \mathrm{~ns}$ \\
Number of bunches / train & 10 \\
Train length & $75.6 \mathrm{~ns}$ \\
Nominal extraction rate & $3.216 \mathrm{MHz}$ \\
Nominal extraction inter-bunch interval & $310.9 \mathrm{~ns}$ \\
\hline \hline
\end{tabular}

Purely periodic bunch extraction is important for testing instrumentation, such as laserwire and cavity BPMs, that operate with a separate resonator circuit. 


\section{Beam stabilization}

\subsection{Intra-train feedback and possible active stabilization}

\subsubsection{ATF2 jitter requirements}

- Goal A - achievement of $37 \mathrm{~nm}$ spot size: The requirement on vertical beam jitter is $<30 \%$ of $\sigma_{y}$. This translates to a vertical jitter at the input to the ATF2 FF optics of less than a few microns.

- Goal B - control of beam position at $\mathbf{n m}$ level: The requirement on vertical beam jitter is $<5 \%$ of $\sigma_{y}$. This translates to a vertical jitter at the input to the ATF2 FF optics of significantly less than one micron.

\subsubsection{Current ATF extraction line jitter situation}

\section{Single-bunch mode}

In single-bunch operation in the current ATF extraction line, jitter of order a few microns has been observed over timescales of several minutes. For example, during the December 2004 operations of the SLAC/LLNL NanoBPM system the r.m.s. vertical beam position was about 3.5 microns. This is believed to be dominated by residual spurious dispersion and energy jitter. Longer-period oscillations, due to known limitations in the cooling water system, yield position variations which can be of order 10 microns over intervals of order 10 minutes. These effects have been found to be more severe during hot-weather running.

\section{Multibunch mode}

The bunchtrain structure employed to date allows for up to 20 bunches, with an inter-bunch interval of 2.8ns, to be extracted from the ATF ring. More serious jitter problems have been encountered in measurements made at the end of the current extraction line. For example, in the December 2004 FONT (Feedback On Nanosecond Timescale [34]) run with 20 bunches per train a jitter buildup along the train was observed, with a strong correlation with the bunch charge. This is consistent with the known fast-ion instability in the ATF ring. At worst pulse-to-pulse jitter of order 100 microns during a run of about one minute was observed for bunches near the end of the train. The best jitter observed was approximately 7 microns r.m.s. for early bunches in the train. In the May/June 2005 FONT run the instability effect was considerably reduced, probably due to improved vacuum in the ring, but was still at the level of 10 microns at the end of the train. 


\section{Conclusions on jitter}

If Goal $\mathbf{A}$ is to be run with one bunch per cycle, the current ATF may be able, on short timescales (of order 1 minute), to meet the jitter specification of a few microns. In order to maintain stability on timescales longer than a few minutes it would be necessary to control effects which currently cause oscillations of greater amplitude.

For multibunch running with the current bunch spacing $(2.8 \mathrm{~ns})$ and number of bunches $(\leq 20)$ intratrain beam feedback would appear to be necessary in order to achieve micron-level stability. A first start at addressing this was made via FONT3/FEATHER feedback system tests in 2004/5. However, the very low latency imposed by the 56ns-long train required compromises to be made on the resolution of the position correction, which is currently limited to about 5 microns at best. Achievement of higher resolution would require a significant re-design and further beam tests. In light of the cold-linac technology choice for ILC such tests are not currently planned.

For Goal B sub-micron stability is required at the entrance to the ATF2 FF. Such a level of stability is beyond the best recorded pulse-to-pulse jitter in the current extraction line setup, even in singlebunch mode. Therefore an intra-train beam-based feedback (and/or feed-forward - see below) system appears to be required in order to stabilise the beam position at the sub-micron level, and by definition this can only work for trains comprising at least two bunches, and ideally three or more bunches, per cycle.

Furthermore, sub-micron stabilisation requires beam position measurement at this level of precision. This will probably require cavity BPMs, for example of the type currently employed in the NanoBPM triplets. However, the need to resolve individual bunches in the feedback system imposes severe restrictions on the cavity ring-down time, which is determined by the cavity $Q$. For ILC-like bunch spacing (order 100ns) bunch-by-bunch position measurement with sub-micron resolution may be possible. However, this would probably require a modified BPM design with lower $Q$, as well as significant effort to process digitally the BPM information in real-time as part of a feedback system with latency matched to the bunch spacing interval.

\subsubsection{Intra-train beam feedback at ATF2}

Two systems can be considered for stabilising the beam upstream of the entrance to the ATF2 final focus:

1. A system using stripline BPM information as the position signal input to the feedback. This will allow potential micron-level stabilisation. This could be achieved by a modification to the FONT3 front-end BPM processor now that a very fast (ns) response is not required for ILC. FONT4 is being designed with a digital signal processor appropriate for a train with ILC-like bunch spacing (c. $150 \mathrm{~ns}$ ); first tests are possible in 2006.

2. A system using cavity BPM information as the position signal input to the feedback. This will allow potential sub-micron stabilisation. The current SLAC/LLNL NanoBPMs have achieved 
position resolution significantly better than $100 \mathrm{~nm}$ and could be considered for use in this system. However, a redesign of the BPM is probably required, as well as a major upgrade to the signal processing, in order to obtain sub-micron-level position information in real time.

At least two bunches are required in order to see any feedback; at least three bunches are required in order to see feedback with a delay-loop. With the planned upgrade of the ATF extraction kicker (October 2005) it should be possible to extract three bunches from the ring with an ILC-like interbunch spacing of 140 or 150ns. This should be adequate for a first demonstration of micron-level beam stability with stripline BPMs, via delay-loop feedback operation, with FONT4 in 2006/7.

Clearly a larger number of bunches would allow more detailed study of the feedback performance. For the planned digital ILC feedback system it would be advantageous to study a number of issues:

- development of algorithms robust against noise and able to cope with non-linearity in the input signal;

- adaptive gain, so as to maintain an updating stable operating point near optimal gain;

- incorporation of feed-forward input information from upstream systems, eg. the damping ring.

with a train of at least ten bunches. This will require significant progress in the design of a fastrisetime extraction kicker in order to extract multiple bunches from the ATF ring with nominal ILC bunch spacing; this is currently being discussed. If feasible, such a system could be installed at the earliest in 2006.

\subsubsection{Ring-to-extraction-line feed-forward system}

A correlation between vertical jitter measured in one BPM in the ATF damping ring and one BPM in the extraction line was first observed with the FONT jitter monitors in June 2004. This offers the possibiity in principle to correct for pre-extraction jitter by using a ring to extraction line feed-forward system. Such a system would need careful planning and design work. For example, it could operate on a bunch-to-bunch basis as an intra-train 'beam flattener', or on a pulse-by-pulse basis. Additionally, it could operate as a standalone system with its own kicker, or as an extra input to the downstream feedback system.

\subsubsection{System integration issues}

The intra-train feedback operation will need to be harmonised with any downstream active stabilisation schemes for the FF magnets, as well as with any additional upstream feed-forward and/or feedback systems. This needs careful thought and planning. 


\subsection{Alignment \& stabilization hardware and procedures}

The hardware and procedures for initial alignment of the magnets and stabilization of the magnet position are described in this section.

In the new ATF2 beam line (downstream of BH4X), there are 37 quadrupole magnets $(28+2$ of them are new), 7 dipole magnets and 5 sextupole magnets. In addition, some horizontal and vertical steering magnets and skew-quadrupole magnets are installed in order to correct the beam orbit and the beam $\mathrm{X}-\mathrm{Y}$ coupling. Tolerances for the initial alignment and stabilization of magnets are being studied now in simulations and will be included as soon as studies are complete.

\subsubsection{Initial alignment of magnets}

Initial alignment will be done utilizing laser trackers and sphere mounted reflectors. Although the tracker has an angular resolution of $5 \mu \mathrm{rad}$ and a distance resolution of $0.63 \mu \mathrm{m}$, changes in temperature and temperature layers will cause lateral and longitudinal refraction which will severely limit the achievable position accuracy. To achieve a position precision of $75 \mu \mathrm{m} / \pm 10 \mathrm{~m}$, targets have to be observed from multiple positions augmented by additional level observations. The resulting measurements need to be analyzed in one integrated least squares fit using the "bundle adjustment" approach. Because the total alignment precision is the sum of the precision of alignment instruments and the precision in the fiducialization of magnetic field center of magnets, the same order of magnitude precision is required in the fiducialization which is described in Section 9.

\subsubsection{Control of position of quadrupole and sextupole magnets}

The position of quadrupole magnets and sextupole magnets is adjusted by cam-type movers [35], which are reused from those installed at the SLAC FFTB beam line. The rotation angle around the beam axis and the position in vertical and horizontal direction in the plane perpendicular to the beam axis are adjusted during beam operation so that the beams pass through the magnetic center of the magnet. Correction values are calculated based on signals from Beam Position Monitors (BPM) and transmitted to each mover.

SLAC cam-type movers have a range of about $\pm 1.5 \mathrm{~mm}$, and a resolution of about $0.04 \mu \mathrm{m}$. There is one rotation of the camshaft for every 40,000 pulses to the motors at a speed of $120 \mathrm{~Hz}$. The speed of motion depends on the rotation angle of the camshaft. The maximum speed of movement is about $30 \mu \mathrm{m} / \mathrm{sec}$.

\subsubsection{Control and Stabilization of the position of the final quadrupole magnets}

The position of the final doublet (FD) quadrupole magnets, QF1 and QD0, must be stabilized to $1 \mathrm{~nm}$ vertically and $50 \mathrm{~nm}$ horizontally in terms of jitter. The most critical position error is the relative motion of the FD and the IP. In order to achieve this stability, the FD and the IP instrumentation 
will be installed on a common rigid support to reduce their relative motion. The first ATF2 run will likely start with this configuration. In this case, only a coarse adjustment (with a resolution of $0.5 \mu \mathrm{m}$ and range of $\pm 0.5 \mathrm{~mm}$, with cam movers) would likely be sufficient.

It is also possible to use active stabilization for later runs. This would be particularly important if the IP were supported independently of the FD, as for ILC. In this case the final quadrupole magnets would be installed on movable supports, which have a very high precision of $1 \mathrm{~nm}$ in the vertical direction and $10 \mathrm{~nm}$ in the horizontal. These supports must have a wide range of movement of $\pm 2 \mathrm{~mm}$. To have this capability, the supports would have two stages; one a coarse cam mover and the other a precision piezo mover. The resolution and the range of movement are $0.5 \mu \mathrm{m}$ and $\pm 0.5 \mathrm{~mm}$ respectively for the cam movers, and $1 \mathrm{~nm}$ and $\pm 0.3 \mu \mathrm{m}$ for piezo movers. To be used in active stabilization, a speed faster than $30 \mathrm{~Hz}$ is required for the piezo movers, because the amplitude of the ground motion of the floor is about $1 \mathrm{~nm}$ at a frequency of $30 \mathrm{~Hz}$. Correction values can be determined from the Beam Position Monitors, or from optical anchors.

\subsection{Ground motion in the ATF and ATF2 areas}

The floor of the ATF damping ring was reinforced using more than 200 piles, $1 \mathrm{~m}$ in diameter and 14 $\mathrm{m}$ in length, when the project was constructed in 1993 [36]. The piles support thick concrete slabs, shielding blocks and the accelerator components. Many expansion joints were introduced to prevent the floor from cracking due to thermal expansion and contraction. The ATF2 extraction beam line is planned to be built outside of the present damping ring area (the ATF area). The floor of the ATF2 area does not have any specially reinforced structure. The ground motion of the ATF2 area was measured and compared with the floor of the ATF beam line to see if there is any significant difference between the floor structures. The measurement results are summarized in the following section. The floor tilt and the ground motion were measured using tilt meters (Leica Nivel 20 digital tilt meters) and acceleration sensors (Tokkyo-Kiki MG-102S) [37]. The measurement locations are indicated in Fig. 7.1 .

\subsubsection{Floor tilt measurements}

Floor tilt was measured for several days in order to see long-term effects such as diurnal motion. Blue lines in Figs. 7.2(a) and 7.2(b) show the floor tilt in the East-West direction for the ATF and ATF2 areas, respectively. The outside air temperature is plotted by red lines in the same plots for reference. An earthquake occurred while taking the data at the ATF beam line, seen as a sharp spike in Fig. 7.2(a). The floor tilt was recovered quickly after the earthquake, leaving no offset in the floor tilt. No clear diurnal effect is observed. On the other hand, the floor tilt in the ATF2 area shows a diurnal effect, which is correlated to the outside air temperature, as seen in Fig. 7.2(b). The peak-to-peak value of this effect is $\sim 10 \mu \mathrm{rad}$ when the outside temperature variation is $\sim 10$ degree. A similar effect is seen in the floor tilt in the North-South direction as well. 


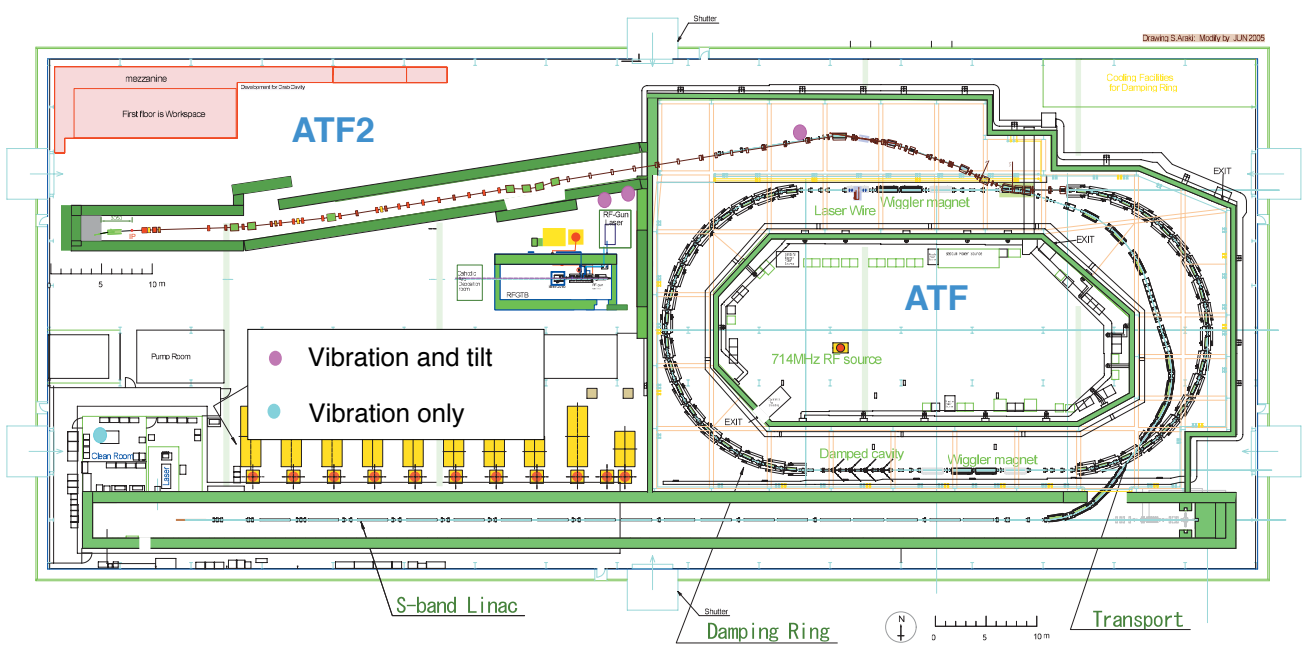

Figure 7.1: Measurement locations for tilt meters and accelerometers.

(a)

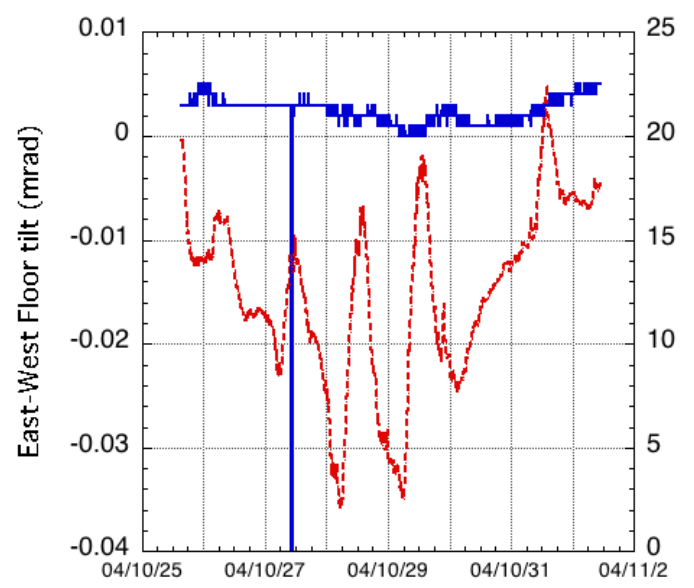

(b)

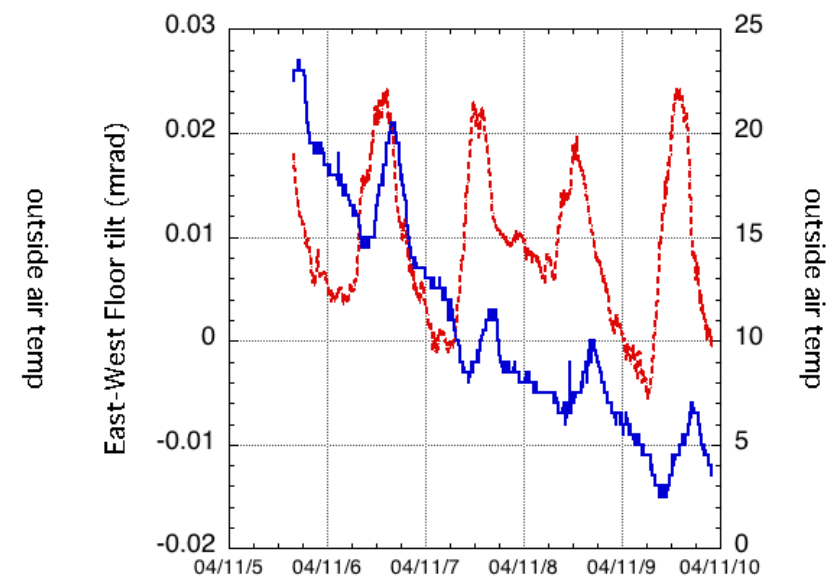

Figure 7.2: Floor tilt measurements in the ATF area (a) and ATF2 area (b). Blue and red lines show floor tilt and outside air temperature, respectively. 


\subsubsection{Vibration measurements}

Ground motion was measured in three locations: in the ATF beam line, in the ATF2 area and in the clean room near the entrance of the ATF building. Fig. 7.3 shows the integrated amplitude in the $\mathrm{X}$ (North-South)-, Y (East-West)- and V (vertical)- directions. It should be noted that the data were taken on different dates. Environmental conditions such as traffic noise may be quite different among these sets of measurements. The ground motion is found to be smallest in the ATF beam line area in all X-, Y- and V- directions. The difference between the ATF area and the other areas is largest in the vertical direction. The integrated amplitude of the vertical motion is $\sim 10 \mathrm{~nm}$ at $10 \mathrm{~Hz}$ at the ATF beam line, while it is an order of magnitude larger in the ATF2 area and in the clean room area. Fig. 7.4 shows the amplitude ratio of the vibration of the girder at the ATF beam line to the floor nearby. The girder has its own natural frequencies and the vibration amplitude becomes larger than that of the floor by an order of magnitude around the natural frequencies.
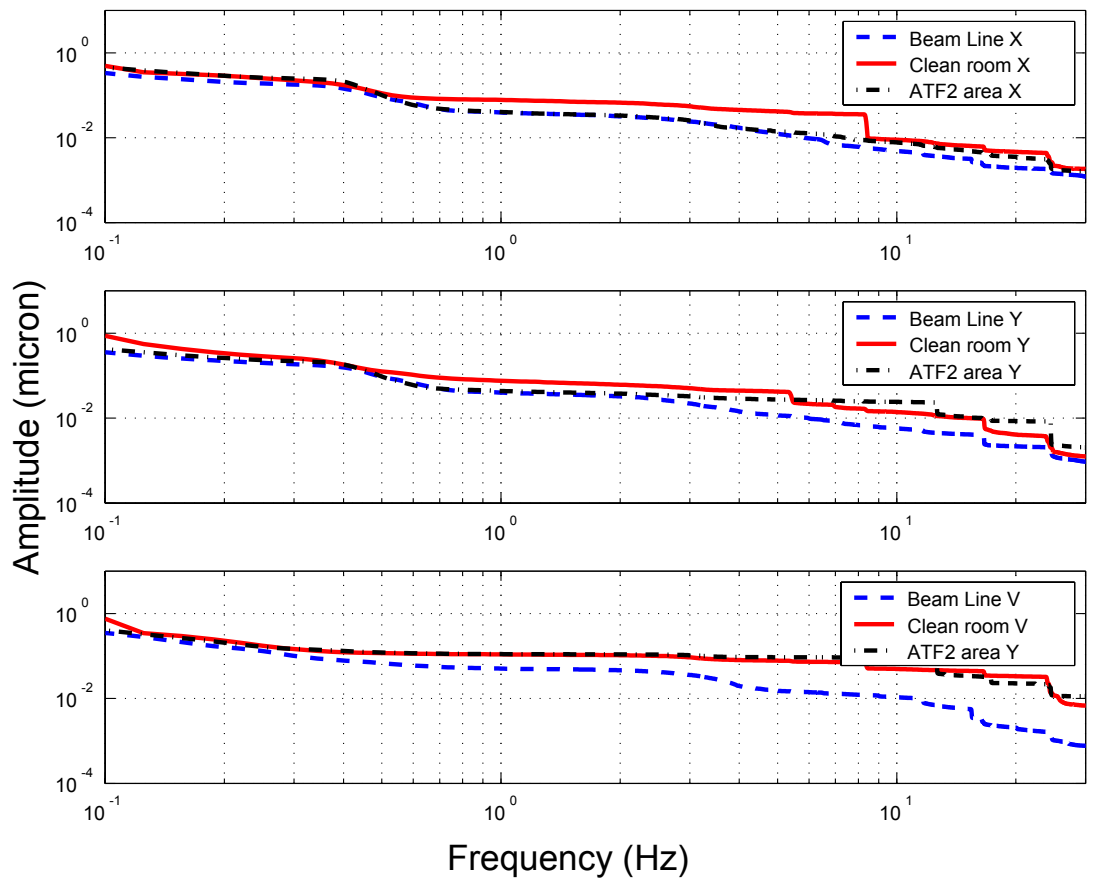

Figure 7.3: Integrated amplitude measured in the ATF beam line, in the ATF2 area and in the clean room. The ground motion is smallest in the ATF beam line, where the floor is reinforced. 


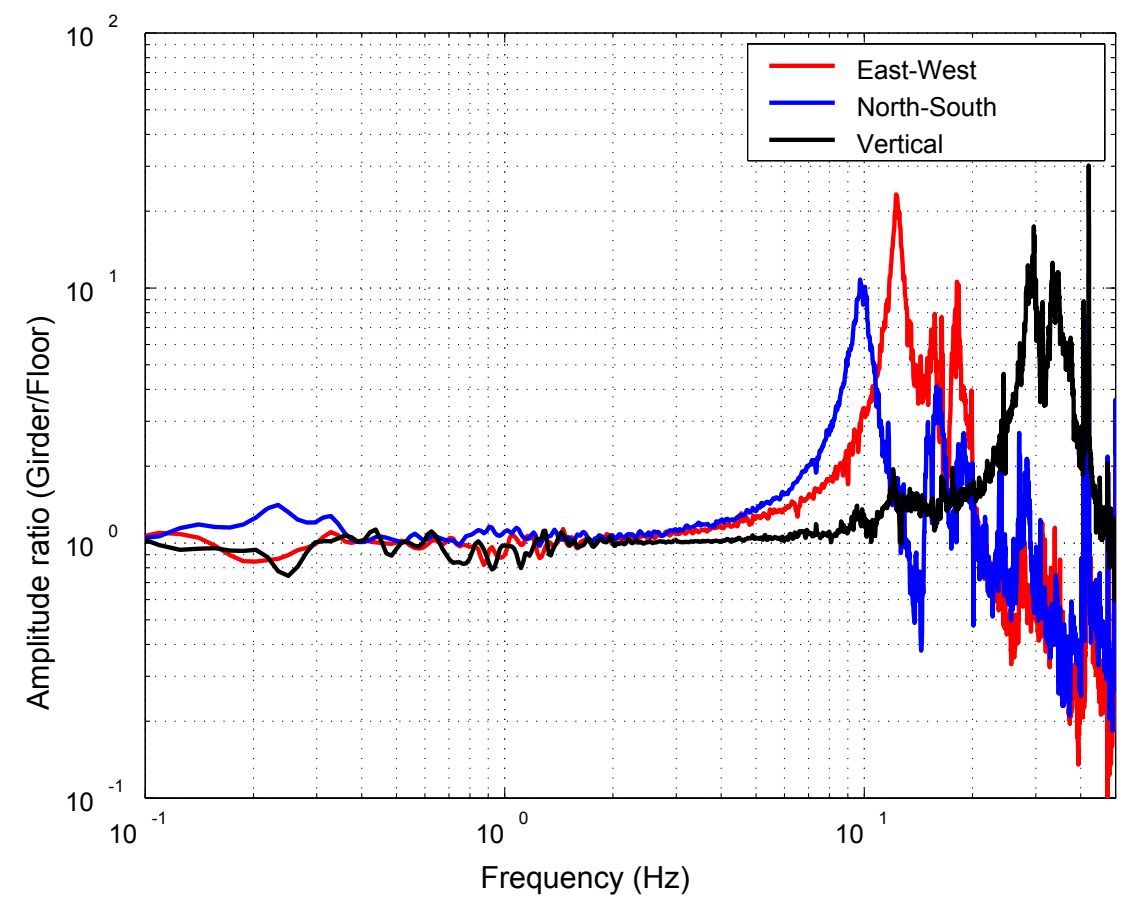

Figure 7.4: Amplitude ratio of the girder motion relative to that of the floor (ATF beam line).

\subsubsection{Summary}

The diurnal effect in the floor tilt is smaller at the ATF beam line than in the ATF2 extraction area. The diurnal effect, which has some correlation with the outside air temperature, may have been damped by the many expansion joints in the ATF beam area floor. The amplitude of the ground motion is observed to be smallest in the ATF area. The difference is most significant in the vertical direction. The reinforcement of the floor in the ATF beam area probably contributed to the damping of the floor tilt and vibration in the ground motion. It is advisable to reinforce the floor in the ATF2 beam extraction area as well. 


\section{Strategy of Commissioning the ATF2 Beam}

The commissioning of ATF-2 can be based on the operational experience gained at the SLC and the FFTB and employ similar procedures as were developed for these two beam lines. The structural differences between the SLC and FFTB optics and the ATF-2 "Raimondi-Seryi" optics will require the use of differently constructed knobs for the latter, but they will not modify the basic philosophy and sequence of the commissioning steps. The commissioning comprises the steering of the beam to the dump, the beam-based alignment of beam-position monitors and magnets, the verification and correction of the linear and nonlinear beam optics, the set up of precision beam diagnostics and feedback systems, and, finally, the tuning of the focal-point (FP) spot size. The reliable provision of a stable low-emittance beam from the damping ring is essential for a rapid commissioning and for achieving the targeted spot size and beam stability.

The basic steps could be (1) alignment without beam, (2) steering and ballistic beam-based alignment with dipole magnets only, (3) optics verification, alignment and correction with dipoles and quadrupoles, (4) dispersion matching and dispersion-free steering, (5) betatron matching and coupling correction of the incoming beam, (6) squeezing or adjusting $\beta^{*},(7)$ chromatic correction, beam-based alignment of sextupoles, optics verification with sextupoles, (8) activating orbit feedback loops across critical regions, (9) FP spot size tuning in regular intervals, (10) continuous monitoring of the optics using jitter data or diagnostics pulses, (11) characterization of the optical properties of the system, and (12) multibunch operation and higher intensity.

It is important that tuning simulations with errors be performed prior to commissioning to understand which level of accuracy is required for the individual tuning steps. Also an online optics model during commissioning ('flight simulator' [38]) will be a great help. In the following we describe the various proposed commissioning steps in greater detail.

(1) Pre-alignment without beam:

Prior to beam operation, all magnets are aligned with a precision of 50-100 $\mu \mathrm{m}$ with respect to a smooth line.

(2) Steering and ballistic alignment with dipole magnets only:

The range of the rf BPMs will be expanded by attenuators. Additional conventional BPMs and screens are useful. The incoming beam orbit should be steered such that it is centered and straight at the start of the beam line. A ballistic beam is then sent between pairs of subsequent dipole magnets to determine the readings of the beam-position monitors for a beam defining a straight line. BPMs with large offsets are afterwards aligned. The strengths of individual dipoles possibly need to be adjusted to keep the ballistic beam close to the center of the beam pipe. Any large dipole roll errors will be evident as vertical deflections. The offsets of the BPMs inferred from the ballistic trajectory are included in the database and subtracted automatically for all subsequent BPM measurements. This ballistic alignment may be conducted in steps, 
switching or keeping off groups of quadrupoles and, possibly, adapting the downstream optics for safe beam extraction to the dump, if beam losses are a concern for the commissioning. A beam profile monitor mounted in front of the beam dump will give a clear indication whether the beams are cleanly extracted. For understanding the properties of the rf BPMs, it might also be instructive to repeat the ballistic beam measurements at various levels of attenuation.

(3) Optics verification, alignment and correction with dipoles and quadrupoles:

The local optics is studied with all quadrupoles and dipoles on, but with sextupoles still off. For the low energy spread of the ATF beam, chromatic effects likely are not a problem except for at the focal point itself. Steering effects of the quadrupoles are minimized by moving their transverse position, until the beam follows the ballistic orbit determined in step (2). If required, more complex beam-based alignment procedures could be applied for the quadrupoles [39]. To characterize the optics, steering magnets are next excited one by one, and the orbit response measured at all beam-position monitors. Position readings taken upstream of the induced steering are used to correct for variations in the incoming beam trajectory. The measured response matrix of all BPMs with respect to all steering correctors is compared with model predictions. If large differences are observed, quadrupole strengths must be adjusted or longitudinal positions in the beam line need to be verified. A modified version of the LOCO code could be employed. In addition, or as alternative, to the steering correctors, the quadrupole magnets could be moved transversely for measuring the BPM response.

(4) Dispersion matching and dispersion-free steering:

The dispersion is measured by injecting a beam at different energies. The beam energy can be varied by changing the rf frequency in the ATF ring. The practical range is $\pm 0.65 \%$, limited by the finite aperture at a high dispersion point $(D=2.5 \mathrm{~m})$ in the present extraction line. Varying the ring rf frequency may also change the beam orbit at the start of the extraction line, if the dispersion at kicker and septum is not zero. The simultaneous generation of energy error and orbit change due to nonzero dispersion in the extraction region is not a problem, because their combined effect is the "beam dispersion" which we need to correct. Once the incoming dispersion has been inferred from the slope of the orbit readings versus the extracted beam energy for BPMs at the start of the beam line, the dispersion match into the final focus can be accomplished with two quadrupoles and two skew quadrupoles located in a region with nonzero dispersion [40]. Such dispersion matching quadrupoles are already available, namely QF3X, QF4X, QS1X and QS2X. After correcting the incoming dispersion, the residual dispersion downstream at other BPMs and the residual orbit errors can be corrected simultaneously using dispersion-free steering with SVD minimization [41, 42].

(5) Betatron matching and coupling correction of the incoming beam:

The incoming Twiss parameters, including coupling, can be measured in the diagnostics section. Four or more normal quadrupoles at the entrance of the final focus are used to adjust the final focus optics to the measured beta and alpha functions. The quadrupoles are varied together and need to be computed iteratively as so-called nonlinear Irwin knobs [43]. Incoming coupling is removed with four skew quadrupoles of the skew correction section. 
(6) Squeezing or adjusting beta-star:

The quadrupoles a the entrance of the final focus also offer the possibility to vary the FP beta functions using Irwin knobs. It might be wise to start the commissioning with a relaxed focusing and go to the design values of $\beta^{*}$ only in a second phase. Whether the actual spot size and demagnification agree with expectation could be controlled at a pre-image point. If the emittance is measured in the diagnostics section, the FP beta function can also be inferred from the beam size at a wire scanner located close to the final quadrupole. For ATF2 two such wire scanners could be mounted on the incoming and outgoing side of the FP, respectively.

(7) Chromatic correction, beam-based alignment of sextupoles, optics verification with sextupoles:

The sextupoles are turned on one by one. For each sextupole, the BPM-corrector response is measured (LOCO style). Once powered, the transverse sextupole positions are scanned. The sextupolar quadratic steering effect allows finding the horizontal and vertical centers of the magnet [44]. Another possible way of aligning the sextupoles is to measure the waist shifts, dispersion, and skew coupling which they introduce depending on their transverse position [45]. After the sextupole alignment, the BPM-corrector response is re-measured. The horizontal and vertical deflections induced by a sextupole when its two transverse positions are varied also allow measuring the $R_{12}$ and $R_{34}$ matrix elements between the sextupole under investigation and sextupole-BPMs downstream. The $R$ matrix between sextupoles is of critical importance to the performance of the system. If a matrix element is found to be larger than tolerable, optics correction, e.g., changes of the strengths of intermediate quadrupoles, will be required. After all elements are aligned, the resolution of the BPMs can be increased and their range decreased.

(8) Activating orbit feedback loops across critical regions:

Once the quadrupoles and sextupoles are aligned with beam-based methods, the BPM centers found by ballistic alignment, the orbit corrected by dispersion-free steering, and the rf BPM resolution increased, the orbits in critical regions must be maintained by slow orbit feedback loops. Critical regions are the area around the FP, the region covering the five sextupoles of the final focus, and the diagnostics section. The feedbacks have to be commissioned and their performance validated. Possible cross talk and cascading between feedback loops has to be understood or implemented. An alternative could be a global feedback for the entire beam line with higher weights assigned to critical elements, like sextupole-BPMs. The feedbacks stabilize orbit and optics throughout the system against drifts on the time scale of minutes, which is a precondition for successful spot-size tuning.

(9) FP spot size tuning in regular intervals:

After controlling alignment and optics in the entire final focus, it remains to tune out residual aberrations at the focal point [46]. This can be accomplished using special orthogonal tuning knobs, which consist, e.g., of transversely moving two or three sextupoles with a fixed relation of step sizes [47]. The maximum range of each tuning knob is limited by additional higher-order aberrations which each knobs introduces, e.g., due to the interleaving of sextupoles [48]. It is therefore important that the upstream optics is well corrected, before commencing the tuning 
with the FP tuning knobs. The tuning knobs can optimize the horizontal and vertical waist, skew coupling, horizontal and vertical dispersion, higher order sextupolar or octupolar aberrations, etc. The tuning has to be repeated in regular intervals (hours). Tuning by a dither feedback which optimizes spot-size related signals as a function of knob step changes may be essential for achieving and maintaining the target spot size. Such a feedback proved indispensable in the last two years of the SLC [49].

(10) Continuous monitoring of the optics using jitter data or diagnostics pulse:

Continuous optics-quality control may be provided in various ways. For the SLC, a scheme was developed to continuously monitor the beam optics by analyzing jitter data [50]. Also, sum readings of certain BPMs signals allowed automatic detection of movements or dipole-strength errors in the SLC chromatic correction region (where $80 \mu$ of diurnal variation were observed). Another possibility could be to inject one or several diagnostics pulses in regular intervals, as had been in use in the SLAC linac [51].

(11) Characterization of the optical properties of the system:

Systematic measurements could characterize the optical properties of the system. This may be useful for comparison with the optics model or for identifying sources of spot-size dilution. The characterization could include measuring the spot size for different incoming beam emittance [52] (vertical emittance can be varied easily in the damping ring; the blow up of the horizontal emittance can possibly be accomplished by mismatch in the ring, or by extracting before reaching the equilibrium emittance), for varying incoming beam energy [52] (by varying the ATF rf frequency), and for different initial orbit position and slope [53]. The spot size can also be quantified as a function of $\beta^{*}$ and beam intensity (correcting for emittance effects) [54].

(12) Multibunch operation and higher intensity:

The magnitude of wake field effects in the ATF-2 final focus can be quantified by studying the intensity dependence of the spot size and of the beam orbit, a notorious concern at the SLC. Multibunch operation may require changes or upgrades to some of the beam diagnostics. 


\section{ATF2 magnets}

\subsection{Introduction.}

The lattice can be found here [55]. The MAD deck of the optics has been transformed into a series of magnets, which start at the present end of the ATF extraction line (the last magnet of which is BH4) and can be divided into 3 sections serving different purposes. The first section starts at QD5X and extends the present extraction line by 19 quadrupoles, the next, matching, section has a chicane made from 4 dipoles and 6 quadrupoles (QMnn) and the last section is the Final Focus which has 16 quads, 3 bends, 5 sextupoles and 2 octupoles. Therefore there are a total of 41 quadrupoles, 7 dipoles, 5 sextupoles and 2 octupoles in the ATF2 beamline.

Some of the quadrupoles are existing magnets that will be moved from other positions in the present ATF extraction line. A total of 30 new quadrupoles, 7 dipoles, 5 sextupoles and 2 octupoles need to be designed and fabricated. In this chapter it will be shown that 28 of the 30 quadrupoles can be made from the same design and a suitable design has been identified.

The list of brand-new magnets (that need to be designed and fabricated) in the optimal beamline is shown in Table. $9.1^{2}$. It has been decided that the maximum energy of the beam in AFT2 will be $1.3 \mathrm{GeV}$ and all the magnets are being designed for this maximum beam energy. The quadrupoles are defined by their $K_{1}$ values, which have dimension of $1 / \mathrm{m}$ and meaning of inverse focusing distance. The relationship between gradient and $K_{1}$ is defined as follows

$$
\text { Gradient }(\text { Tesla/meter })=K_{1} \times(B \rho) /(\text { effective length }) \text {. }
$$

Where $(B \rho)=$ Beam Energy $/\left(\right.$ speed of light $\left.\mathrm{x} 10^{-9}\right)=1.3 / 0.2997925=4.3363$ Tesla*meter.

\subsubsection{Choice of magnets' effective length and apertures.}

To turn any lattice into physical magnets we need to define their effective lengths and their apertures, where the beam passes. We need to make the magnets short enough to leave room for quite sizable cavity Beam Position Monitors (BPM). This led to a range of between $0.1 \mathrm{~m}$ and $0.25 \mathrm{~m}$ for the quads' effective length. The quad apertures must be sufficient to pass a +/-1\% energy spread $1.30 \mathrm{Gev}$ beam: around $15 \mathrm{~mm}$ radius. There is some flexibility in the $15 \mathrm{~mm}$ value and we need to leave additional room for a beam pipe. So we have specified that the radius of any existing quad style that we might assign to the FF quads must be at least $16.0 \mathrm{~mm}$. Noting that the proposed BPMs (described in Section 4.1) have an $18 \mathrm{~mm}$ diameter, this minimum $16.0 \mathrm{~mm}$ radius (=32 $\mathrm{mm}$ diameter) will ensure the BPMs can be accommodated in the quads.

\footnotetext{
${ }^{2}$ Comment on numbering system of the magnets. As the FF lattice has evolved we have ended up with some magnets with exactly the same name, e.g. two QF9s, two QD4s. They are intended to be separate magnets.
} 
Table 9.1: List of new magnets in the optimal beamline.

QUADRUPOLES

\begin{tabular}{cc}
\hline Name & Length $(\mathrm{m})$ \\
\hline
\end{tabular}

Extension of ATF extraction line

$\begin{array}{ccc}\text { QD7X2 } & 0.2 & -0.663296888238 \\ \text { QD9X2 } & 0.2 & -0.663296888238 \\ \text { QF9X2 } & 0.2 & 0.6632968888238 \\ \text { QD10X2 } & 0.2 & -0.663296888238 \\ \text { QF10X2 } & 0.2 & 0.663296888238 \\ \text { QD11X2 } & 0.2 & -0.663296888238 \\ \text { QF11X2 } & 0.2 & 0.663296888238 \\ \text { QD12X2 } & 0.2 & -0.663296888238\end{array}$

Matching section

$\begin{array}{llc}\text { QM16 } & 0.2 & 0.321774055234 \\ \text { QM15 } & 0.2 & 0.475635506536 \\ \text { QM14 } & 0.2 & -1.1 \\ \text { QM13 } & 0.2 & 0.764858104453 \\ \text { QM12 } & 0.2 & 0.744548379793 \\ \text { QM11 } & 0.2 & 0.0\end{array}$

Final Focus

\begin{tabular}{ccc} 
QD10 & 0.2 & -0.313548300101 \\
QD10 & 0.2 & -0.313548300101 \\
QF9 & 0.2 & 0.374074652373 \\
QF9 & 0.2 & 0.374074652373 \\
QD8 & 0.2 & -0.560633553863 \\
QF7 & 0.2 & 0.5995239085 \\
QD6 & 0.2 & -0.560633553863 \\
QF5 & 0.2 & 0.374074652373 \\
QF5 & 0.2 & 0.374074652373 \\
QD4 & 0.2 & -0.313548300101 \\
QD4 & 0.2 & -0.313548300101 \\
QD2B & 0.2 & 0.588908646492 \\
QF3 & 0.2 & -0.2494251727 \\
QD2A & 0.2 & -0.274411188136 \\
QF1 & 0.4 & 0.913516557464 \\
QD0 & 0.5 & -1.494423007975 \\
\hline
\end{tabular}

\section{DIPOLES}

\begin{tabular}{lll}
\hline Name & Length $(\mathrm{m})$ & Bend Angle (rad) \\
\hline
\end{tabular}

Chicane

4 dipoles, each $0.8 \mathrm{~m}, 0.186$ radians

Bends

\begin{tabular}{lll} 
B5 & 0.8 & 0.05108 \\
B2 & 0.8 & 0.03439 \\
B1 & 0.8 & 0.04338 \\
\hline
\end{tabular}

SEXTUPOLES

\begin{tabular}{ccc}
\hline Name & Length $(\mathrm{m})$ & $K_{2}\left(1 / \mathrm{m}^{2}\right)$ \\
\hline SF6 & 0.2 & -12.302831248769 \\
SF5 & 0.2 & 0.146523171202 \\
SD4 & 0.2 & -16.224619020245 \\
SF1 & 0.2 & 3.38989381939 \\
SD0 & 0.2 & -4.986044573043 \\
\hline
\end{tabular}

\section{OCTUPOLES}

2 magnets, strength not yet defined 


\subsection{Performance Requirements of the ATF2 Magnets.}

The new ATF2 magnets have 7 requirements they must meet: (1) integrated magnetic strength, (2) physical dimensions of core and aperture, (3) compatibility with SLAC's FFTB magnet movers (see more about movers in Section 9.8 below), (4) relative field errors which determine the stability requirements of the magnet power supplies [in addition, if their currents and voltages could be made to be compatible with SLAC's FFTB switching power supplies : 40 volts, 250 amps, and if we could use these old PS, it would save us some money] (5) compatibility with new cavity Beam Position Monitor [described in Section 4.1], (6) field quality as described by multipole content being below certain values, especially the sextupole component in the quads and (7)(for the quads only) for Beam Based Alignment (BBA) purposes the field must be able to be changed by $4 \%$ in less than 5 seconds without producing eddy currents that last more than a few seconds beyond the end of the current ramp.

The field quality and relative field error tentative values are given in Section 3 of this proposal. The new ATF2 magnets must meet all these requirements else the beamline will not be useful.

\subsection{Acquisition of the ATF2 magnets.}

Our initial approach to acquiring these 41 magnets, to minimize their cost, was to look at SLAC's inventory of not-being-used magnets that were designed, built and run in the Stanford Linear Collider's (SLC) beamlines, to see if any are close-enough matches in aperture, length and integrated strength. The SLC beamlines that are presently not in use (nor ever likely to be run again) are those that transported the $\sim 50 \mathrm{GeV}$ electron and positron beams from the Beam Switchyard to the beginnings of the (infamous) arcs, out of the arcs into the e+ and e- final focus, the SLC FF themselves and past the interaction point into the e+ and e- dumps. Because they were transporting $\sim 50 \mathrm{GeV}$ beams all these magnets tend to be much stronger than we need for the $1.30 \mathrm{GeV}$ ATF2 FF.

Nevertheless, we have carefully looked through the specifications (and some magnetic measurements) of all the quads, bends and correctors in the above-mentioned SLC beamlines and tried to make the best matches we could with the 22 quads and 7 bends. There were no satisfactory matches. The SLC magnets were either too long, or their apertures were too small or they were too strong. For most of the quads the SLC magnets are 5 to 10 times stronger than they need to be, so they would possibly be running out of their linear regime. The old SLC sextupoles and octupoles were not suitable either.

Our colleagues at CERN also looked through their "spare" magnet inventory to see if they had any suitable magnets, they did not.

Our next approach was to look for existing designs of magnets that would fulfill the 7 requirements listed above. If we could find an existing magnet design then we could use its existing drawings to fabricate new magnets and this would save some money and lots of time. 


\subsection{Choice of Existing Quadrupole Design for most of the ATF2 Quads.}

We considered several existing quadrupole designs at SLAC (e.g. PEPII injector, SPEAR transport) and there was nothing suitable. So we looked at some KEK quads, several designs that are used in various places in the ATF. We were successful in finding a design that we believe can be the design for ALL the ATF2 quads except the final doublet, QF1, QD0 [which will be designed from scratch]. This is the TOKIN 3390B quadrupole design that is used for some of the btq transport line quads, for example, QB4 and QB5. It is also called the QICB style. Be aware that there are 3 very similar quad magnets made by TOKIN for the ATF, they have exactly the same core but different coils. One version has 67 turns per coil, another has 27 turns per coil and the version we have chosen has 49 turns per coil. It also has trim coils with 20 turns each.

All the pertinent details, including 2 drawings and some photos about the 3390B quad (as we shall call it) are given in the next subsection.

\subsubsection{Details of the TOKIN 3390B style quad chosen for the new ATF2 quads.}

The TOKIN 3390B has a $32 \mathrm{~mm}$ bore diameter, its solid, low carbon steel, core is $180 \mathrm{~mm}$ long, so its nominal effective length is $196 \mathrm{~mm}$. The poletips have a hyperbolic shape. Its core is $470 \mathrm{~mm}$ wide and high and the magnet's overall length is $274 \mathrm{~mm}$. So it meets the physical dimensions requirement. The main coils are made from "OFC-2" copper conductor, $6 \mathrm{~mm}$ square with a $4 \mathrm{~mm}$ diameter cooling hole. They have 49 turns, wound in a racetrack shape. Along one side of the main coil there are 20 turns of a trim coil, made from sold copper conductor $2.5 \mathrm{~mm}$ in diameter. The 2 sets of turns are potted into one mass. "Snapshots" of this magnet's top assembly drawing are shown in Fig. 9.1 and 9.2 .

The $3390 \mathrm{~B}$ was designed for a nominal current of $90 \mathrm{amps}$, at which the magnet voltage is 9.25 volts and the total water flow is 1.25 liters/minute (with an unknown water pressure drop). At 95 amps the gradient produced is 45.68 Tesla/meter, as we will see below, this means this design can generate all the integrated gradients needed in the ATF2 quads (not considering QD0 and QF1) in the "optimal" configuration. The matching quads, QM11 through QM16, have varying strengths and we are informed that the maximum K1 value a matching quad may need to reach is 2.5 . This is a gradient of $54.2 \mathrm{~T} / \mathrm{m}$ in this design. A $2 \mathrm{D}$ computer POISSON model of this design was made and it showed that 112.8 amps will be needed to reach $54.2 \mathrm{~T} / \mathrm{m}$. The rise in the water temperature at this current with a delta $\mathrm{P}$ across two water circuits of $6 \mathrm{Kg} / \mathrm{cm}^{2}$ will be $8.8^{\circ} \mathrm{C}$, this is acceptable. Therefore this design meets the magnetic strength requirement for the ATF2 quads. Fig. 9.3 shows two photos of one of these TOKIN 3390B quads in an ATF beamline.

In order to fit in with the new cavity BPM, the space between the coils at the core face must be at least $100 \mathrm{~mm}$ across, and the coil ends must not protrude more than $70 \mathrm{~mm}$ beyond the core end, this design meets both requirements.

Each quadrupole in the final focus part of the ATF2 will sit on a magnet mover. We are planning to borrow sufficient magnet movers from the SLAC FFTB beamline when it is dismantled in the 


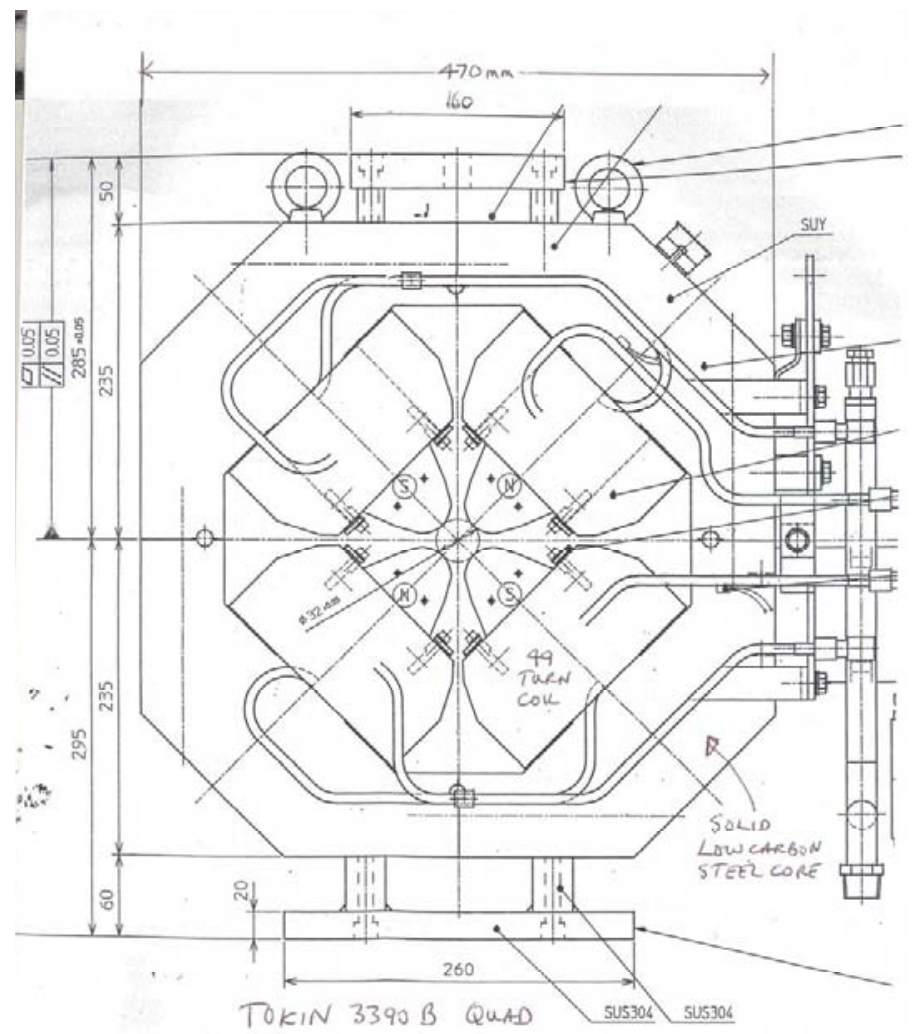

Figure 9.1: Snapshot of top assembly drawing of the TOKIN 3390B quad.

summer of 2006, more details about these movers are given in a section below. The shape of the TOKIN 3390B core is compatible with a FFTB magnet mover. Special V blocks will be added to the magnet and extra brackets for the LVDT sensors to touch. Each magnet weighs $270 \mathrm{~kg}$, this is well within the weight capability of the mover. So the TOKIN 3390B design meets the requirement of being compatible with the SLAC FFTB magnet movers.

\subsubsection{Field Quality of the TOKIN 3390B quadrupole design.}

The field shape quality requirements have been worked out by various beam physicists and their present tentative values are listed in Section 3 of this proposal. The field shape tolerances are tightest in the sextupole/quadrupole ratio, the actual values vary from quad to quad. The tightest is $0.04 \%$ at radius of $10 \mathrm{~mm}$ (not counting the final doublet). It is difficult to measure the sextupole component accurately in a small bore quad. One has to make a special effort with the design and fabrication of the rotating measuring coil. The TOKIN 3390A/B/C quads were measured before they were installed with some unknown device that produced values for the normal and skew components of the multipoles from $n=1$ (dipole) to $n=10$ (20-pole). We have transformed them into "bn" coefficients quoted as fractions of the quadrupole field at $10 \mathrm{~mm}$. The measured values for the "unallowed" multipoles, e.g. 


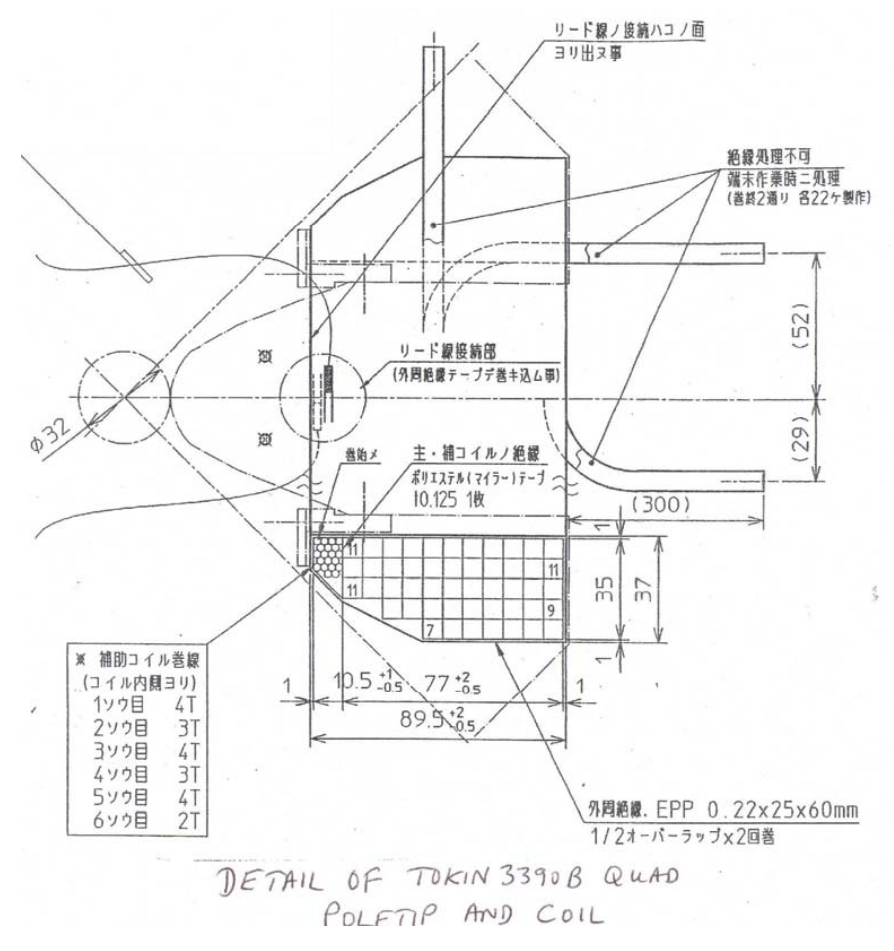

Figure 9.2: Snapshot of coil drawing of the TOKIN 3390B quad.

the sextupole and octupole components, are not consistent from quad to quad, whereas the allowed 12-pole values are.

We cannot tell if these variations are real- and come from fabrication errors in these magnets, or if they are caused by the measuring apparatus. Nevertheless some of the "btq" quads beat the $0.04 \%$ tolerance, and as it is hard to inadvertently measure a sextupole to be smaller than it really is, therefore we believe that we can make quadrupoles using the TOKIN 3390B design that will meet the field quality requirements. We will add tight extra dimensional tolerances to some of the core parts to ensure the sextupole component is small enough.The 12-poles will easily meet the ATF2 tolerances.

\subsection{Meeting the Relative Field Errors Requirements and Power Supplies.}

The field stability over time tolerances have been calculated by various beam physicists and some tentative values are listed in Section 3 of this proposal. They vary quite widely over the FF quads and bends. e.g. for QD10 is 70 ppm, for QF3 is $8822 \mathrm{ppm}$; the 3 bends, B1, B2 and B5 have the tightest stability tolerances: 18,24 and $37 \mathrm{ppm}$ respectively. These tolerances are for keeping various changes in the beam parameters below $2 \%$. The time stability of the magnets depend on the stability of their power supplies. 

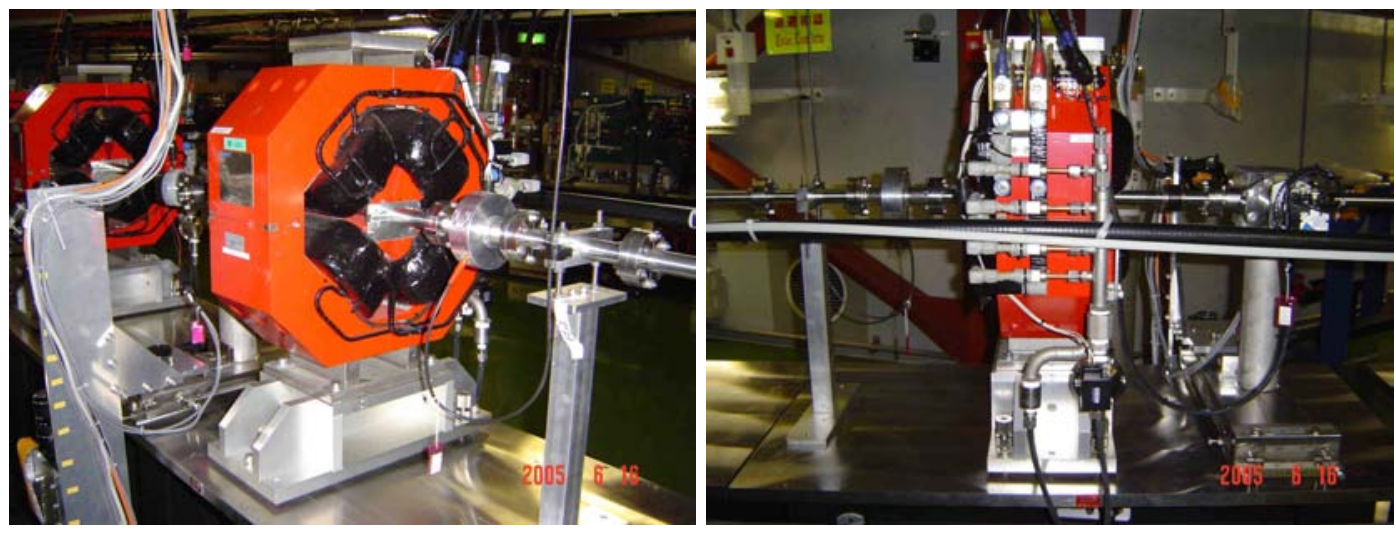

Figure 9.3: PHOTOS of a TOKIN 3390B quadrupole in use at ATF, KEK.

We have been looking into borrowing the switching power supplies that were designed and made for the FFTB at SLAC about 12 years ago. There are 34 rack mounted supplies rated at 40 volts and 250 amps. The possibility of getting these power supplies for ATF2 is currently under investigation. Nevertheless it is instructive to analyze these PS's capabilities to understand the requirements on the ATF2 magnet power supplies.

These PS come with a SLAC-designed controller and feedback circuit that make them have a (measured) 10 ppm stability at 250 amps. As the PS is run at lower currents its stability degrades according to the formula: 10 divided by the fraction of 250 amps the output current represents. e.g. if output current is 25 amps, the fraction is $25 / 250=0.1$, and the stability is $10 / 0.1=100 \mathrm{ppm}$. We calculated the current each ATF2 quad would run at if it were made from the TOKIN 3390B design (and the 3 bends made from another ATF design) and calculated the stability of the FFTB PS at that current, then we compared that with the required stability. The results are shown in the Table 9.2.

The currents and voltages of the ATF2 magnets can be generated by the FFTB PS.

The FFTB PS system could be modified to lower the current corresponding to the 10 ppm stability: according to the engineer who designed the controller and feedback circuit, we could remove the single copper bus bar in the transductor and replace it with several turns of wire in order to reduce the value of the current corresponding to the $10 \mathrm{ppm}$ stability. E.g. put 5 turns in the transductor core and full scale current would become $250 / 5=50$ amps. Each transductor could have a custom number of turns to give optimum matching between PS and magnet load.

So, if we can borrow the FFTB PS system we can make them meet our tight tolerances. Otherwise we will procure new PS from industry which match the magnets' current needs and the stability tolerances. 
Table 9.2: Achievable magnet stability if (unmodified) FFTB power supplies are used. Here $I_{o}$ is operating current with suggested ATF magnet design. $\triangle B / B_{F F T B}$ - PS stability at the operating current if a 250amp FFTB PS is used. $\triangle B / B$ - Tolerable relative field error. Magnets showing in italic do not meet their published stability tolerance if powered by FFTB power supplies.

\begin{tabular}{ccccc}
\hline Q or B & T/m or B.dl & $\begin{array}{c}I_{o} \\
\text { name }\end{array}$ & $\begin{array}{c}\Delta B / B_{F F T B} \\
\text { Tpm }\end{array}$ & $\begin{array}{c}\Delta B / B \\
\text { ppm }\end{array}$ \\
\hline & & & & \\
Quadrupoles & & & & \\
QD10 & 6.8033 & 14.15 & 177 & 70 \\
QF9 & 8.103 & 16.85 & 148 & 300 \\
QD8 & 12.13 & 25.23 & 99 & 320 \\
QF7 & 13.0 & 27.04 & 93 & 250,000 \\
QD6 & 12.13 & 25.23 & 99 & 320 \\
QF5 & 8.103 & 16.85 & 148 & 300 \\
QD4 & 6.8033 & 14.14 & 177 & 70 \\
QD2B & 12.74 & 26.5 & 94 & 468 \\
QF3 & 5.417 & 11.3 & 221 & 8822 \\
QD2A & 5.937 & 12.35 & 202 & 2450 \\
QM16 & 6.976 & 14.51 & 172 & 4819 \\
QM15 & 10.313 & 21.45 & 117 & 5075 \\
QM14 & 23.05 & 49.60 & 50 & 350 \\
QM13 & 16.583 & 34.49 & 73 & 7700 \\
QM12 & 16.143 & 33.57 & 75 & 632 \\
QM11 & ZERO & - & - & - \\
QD10X (new extrc) & 14.381 & 29.91 & 83.3 & N.A. \\
QM14 with K1=2.5 & 54.2 & 112.8 & 22.6 & 350 \\
& & & & \\
Dipoles & & & & \\
B1 & 0.1881 & 84.8 & 30 & 18 \\
B2 & 0.15134 & 68.2 & 37 & 24 \\
B5 & 0.22151 & 99.9 & 25 & 37 \\
\hline & & & &
\end{tabular}




\subsubsection{Meeting the Eddy Current and BBA Requirements.}

The third performance parameter we must worry about are eddy current effects that happen when the current is changed quickly: eddy currents will be induced in a solid steel core and they might be large enough to slow down the changing of the field in the bore. Experiments that were done some years ago on a small NLC main linac, solid steel, quad indicate that one must place a limit on the rate of change of the current during a Beam Based Alignment (BBA) process - but the rate will still be fast enough to meet the timing requirements of the BBA. Recently experiments were done on a larger quad at KEK, and the level of eddy currents observed were acceptable. Conclusion is that we can change a solid core quad fast enough for ATF2 tuning and Beam Based Alignment procedures, the exact ramping rate of the current will be established by experiment on the first fabricated magnet. It is not necessary for the cores to be laminated and so the TOKIN3390B solid core is acceptable.

\subsection{Procurement of the ATF2 quadrupole magnets: potential vendor and schedule.}

The plan of our collaboration is that while SLAC and KEK produce the design outlines, IHEP (Institute of High Energy Physics, Beijing) would be responsible for actual magnet manufacturing which is scheduled to begin in the autumn of 2005 .

Field measurements of the quadrupole magnets require a properly bucked measuring coil. For this purpose, an adequate double coil of the correct diameter for ATF2 quadrupole magnets will be built by the IHEP group.

\subsection{Choice of a design for the ATF2 chicane dipoles and FF bends.}

A search similar to the one we did for a quad design has been made for an existing DIPOLE design for the FF bends and chicane bends. An ATF dipole made by Sumitomo, with a drawing number RD1233, gap of $32 \mathrm{~mm}$, curved poletip, effective length of $0.79778 \mathrm{~m}, 36$ turns per coil, has been identified as a reasonable candidate. It produces a bend angle of 0.187 radians in a $1.3 \mathrm{GeV}$ beam with 360 amps. Its basic parameters fit the requirements of the ATF2 FF bends, we will finalize the details later in the year.

The above tolerance table shows that, if these 3 FF bends have to run off a 250 amp FFTB PS they will almost meet the very tight stability tolerances. They would need 84.8, 68.2 and 99.9 amps. We have not considered any field shape tolerances yet - they have not been published for any bends yet.

There are some new chicane dipoles, presently specd to generate a 0.186 radian bend angle for a 1.3 $\mathrm{GeV}$ beam. If we made them like the Sumitomo dipole theyd need about $360 \mathrm{amps}$ to reach this $10.6^{\circ}$ bend angle. Obviously the 250 amp FFTB PS wouldn't work, the other switching PS used in the FFTB reaches a maximum current of 333 amps - again, less than the 360 amps we need for the $10.6^{\circ}$. But, we're told this was a somewhat arbitrary choice of angle, it provides an offset of $30 \mathrm{~cm}$ away 
from the straight ahead beam direction for some diagnostic equipment in order to use old FFTB PS for these chicane dipoles we need this $30 \mathrm{~cm}$ to be reduced by about $10 \%$, we think this can be done.

\subsubsection{More information on the FFTB Magnet Power Supplies.}

The FFTB power supplies and their controlling system were custom designed at SLAC. They are described in details in Ref. [56].

The supplies were made in the early 1990s and have been running successfully at the FFTB since (but not continually as this is a sporadically used test beam). They are quite reliable, but somewhat difficult to troubleshoot when they do fail.

\subsection{Information on the SLAC FFTB Magnet Movers.}

Each quadrupole in the FF of ATF2 will sit on a magnet mover. We are planning to borrow sufficient magnet movers from the SLAC FFTB beamline when it is dismantled in the summer of 2006, more details about these movers are given in Ref. [57]. The movers are fully-automated and capable of positioning magnets weighing up to $600 \mathrm{Kg}$ to a few microns over several millimeters.

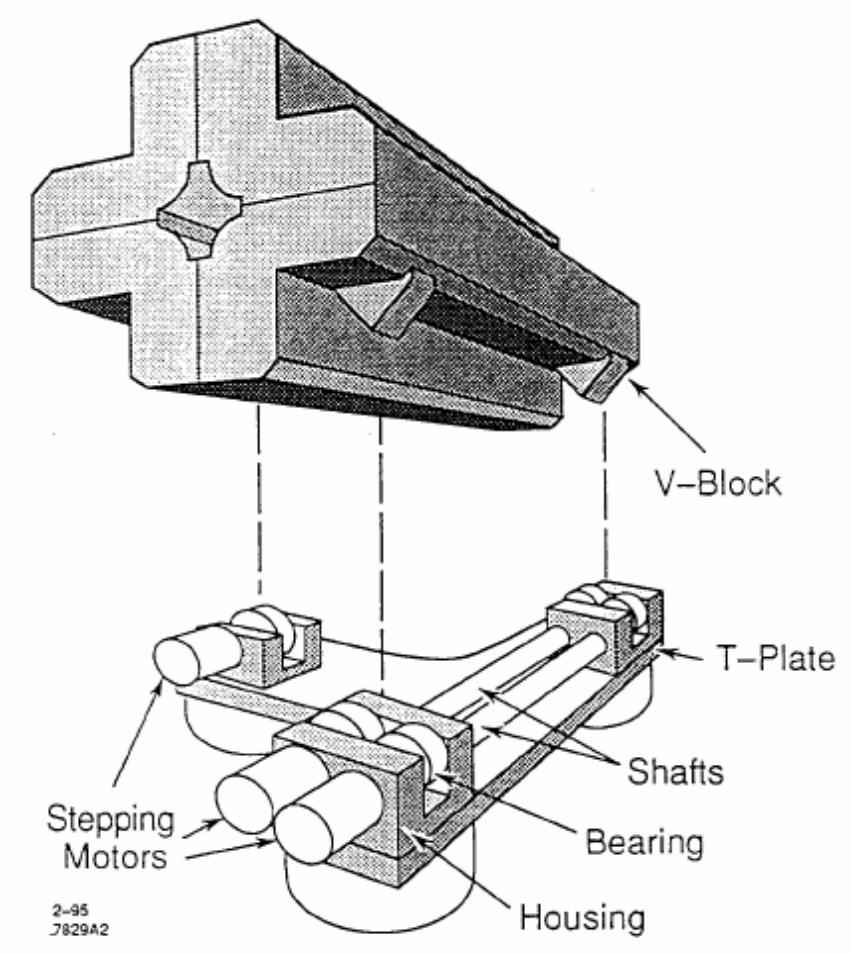

Figure 9.4: FFTB magnet mover. 
The shape of the TOKIN 3390B core is compatible with a FFTB magnet mover. Special V blocks will be added to the magnet and extra brackets for the LVDT sensors to touch. Each magnet weighs $270 \mathrm{Kgram}$, this is well within the weight capability of the mover.

A spare magnet mover and controls has been sent to KEK for scientists to become familiar with its features and controlling system. A SLAC FFTB MAGNET MOVER, showing how it interfaces with a quadrupole is shown in Fig. 9.4. 


\section{ATF DR performance with ILC train}

\subsection{Train format and emittance in ATF}

ATF (Accelerator Test Facility) at KEK [58] has been originally established to demonstrate the superlow emittance multi-bunch electron beam for Linear Collider based on the warm technology. ATF consists from 1.5 GeV Linac, a beam transport line, 1.5 GeV DR, and a extraction line for diagnostic study.

The beam source is a S-band RF gun with CsTe cathode driven by a multi-bunch UV laser [59]. The time structure of the beam is 20 bunches with $2.8 \mathrm{~ns}$ spacing. By manipulating the laser time structure, number of bunches in a train can be changed from 1 to 20 . The bunch spacing, 2.8 ns is fixed because it is determined by Mode-lock frequency of the laser cavity, $357 \mathrm{MHz}$. This multi-bunch beam is accelerated up to $1.3 \mathrm{GeV}$ by the linac and injected into DR with a kicker which has $60 \mathrm{~ns}$ flat top. The extraction is done with the same scheme. Parameters of the ATF injected beam are summarized in Table 10.1

Table 10.1: Parameters of the injected beam.

\begin{tabular}{ll}
\hline \hline Beam energy & $1.3 \mathrm{GeV}$ \\
Bunch population(Max.) & $3 \times 10^{10}$ electrons \\
Bunches/train & $1-20$ \\
Bunch spacing & $2.8 \mathrm{~ns}$ \\
Emittance (norm.) & $<10 \times 10^{-6} \mathrm{~m}$ \\
\hline \hline
\end{tabular}

ATF DR is a race-track shape storage ring with $138.6 \mathrm{~m}$ circumference. The combined bend lattice is employed to minimize the horizontal dispersion in the bending field. Because ATF is originally constructed as a prototype of DR for a LC based on the warm technology, the beam handling scheme is different from that of ILC based on the cold technology. As explained, the beam is always handled with an identical multi-bunch form in the injector linac, DR, and the extraction line. On the other hand, the beam is manipulated bunch by bunch in ILC. The beam is extracted from DR bunch by bunch independently in ILC because the bunch is stored with a compressed bunch format, shorter bunch spacing. Generating ILC format beam is therefore a big issue for ATF-DR in ATF2 project.

Ring RF is $714 \mathrm{MHz}$ giving 330 harmonic number and 165 buckets with $2.8 \mathrm{~ns}$ spacing. All buckets can not be filled because room for the rise and fall time of the injection and extraction kickers are necessary. Three trains, totally 60 bunches, has been stored successfully without any bucket loss [60]. In the full filled mode, each train is separated with $100.8 \mathrm{~ns}$ spacing. In the single bunch mode, i.e. one bunch in a train, three bunches are stored in DR with $154 \mathrm{~ns}$ spacing. If these three bunches are 
extracted with an extraction kicker with a long flat top which is enough to extract the whole buckets in DR, three bunches with 154 ns spacing are obtained in the extraction line. This is an idea to make an ILC like beam from ATF.

Even though the long pulse kicker can make the semi-ILC bunch format, it is totally different system than that in ILC. The fast kicker expected in ILC is operated $3 \mathrm{MHz}$ with a short rise and fall time less than 20 ns at least. Since the space to install such fast kicker is very limited in ATF and the fast kicker is not established yet, a feasibility study has to be done before the fast kicker is installed into ATF-DR. From January 2004, an experiment to demonstrate a proof of principle of the fast kicker based on the strip-line electrode chamber is started. Detail of these kicker R\&D works are explained in Section 6.1.

The horizontal and vertical emittance (normalized) were measured to be $2.8 \times 10^{-6} \mathrm{~m}$ and $1.0 \times 10^{-8} \mathrm{~m}$ respectively in 2003 by the laserwire beam size monitor [25]. The vertical emittance is even lower than that in ILC, $2.0 \times 10^{-8}$. The emittance is also measured after the beam is extracted into the diagnostic beam line with the wire scanner beam size monitor [2]. According to this measurement, the vertical emittance is $3.0 \times 10^{-8} \mathrm{~m}$ which corresponds to three times of that in DR. This discrepancy is considered to be due to a coupling effect between X-Y motion in the extraction line.

Several possible sources of the X-Y coupling are considered. One is an improper non-linear field in the extraction kicker and/or in the septum magnet, which can not be corrected by a skew Q magnet. Another possibility is a field error in the skew Q magnet in the extraction line. To diagnose the source of the coupling in the extraction line, a stable beam is required as a prove. On the other hand, the extracted beam has a jitter approximately $50 \%$ of the beam size. The reason of the orbit fluctuation is considered to be incompleteness of the orbit jitter compensation in the double kicker system and the energy fluctuation of the stored beam in DR through the spurious dispersion in the extraction line.

According to several studies, the source of the energy fluctuation in DR is unusual synchrotron oscillation. Sakanaka [61] pointed out that even small noise in the ring RF causes a large synchrotron oscillation through the coupled bunch effect. To cure this pathology, two methods are considered. One is a feed-forward control for the extracted beam orbit. A clear correlation has been observed between the DR orbit before the extraction and the orbit in the extraction line. By using this correlation, the beam orbit is kicked by some fast magnet in the extraction line to compensate the orbit fluctuation. The second method is damping the synchrotron oscillation in the DR by using a feedback control on the ring RF. Ross and Meller carried out a basic experiment in ATF-DR to damp the oscillation with a feed-back circuit [62]. In the system, phase of the BPM pickup signal to a reference signal is detected by a mixer. The ring RF phase is controlled by this feed-back signal. As a result, the oscillation amplitude was successfully compensated.

The emittance dilution due to the X-Y coupling and the orbit jitter should be solved not only for ATF2, but also for ILC injector prototype. As mentioned, ATF has already achieved the normalized vertical emittance required from ILC. Even though, ATF does not succeeded to extracted such high quality beam without dilution. ILC has a very tight limitation for the orbit jitter of the extracted beam from DR. The beam extraction and transportation itself are very important issues for ILC DR 
Table 10.2: Parameters and achieved performance of ATF-DR.

\begin{tabular}{ll}
\hline \hline Circumference & $138.6 \mathrm{~m}$ \\
RF frequency & $714 \mathrm{MHz}$ \\
Harmonic number & 330 \\
Normalized emittance $(\mathrm{x} / \mathrm{y})$ & $2.8 \times 10^{-6} / 1.0 \times 10^{-8} \mathrm{~m}$ \\
Geometrical emittance $(\mathrm{x} / \mathrm{y})$ & $1.1 \times 10^{-9} / 4.0 \times 10^{-12} \mathrm{~m}$ \\
\hline \hline
\end{tabular}

development.

In this context, the geometrical emittance is a more reasonable index to qualify the beam performance because the emittance dilution might not be scaled with the beam energy as the normalized emittance. It would be better if the $2.0 \times 10^{-12}$ geometrical emittance required as ILC DR is demonstrated in ATF-DR. Since the geometrical emittance achieved in ATF-DR is $4.0 \times 10^{-12} \mathrm{~m}$, it should be decreased to half of the current achievement.

ATF-DR emittance is determined by the residual vertical dispersion. The vertical dispersion is reduced by the tuning procedure so called "Dispersion Correction" established by Kubo [2]. One way to get more accurate "Dispersion Correction" is improving BPM resolution. The current BPM resolution is $2 \mu \mathrm{m}$ which is near the limit, but there is a possibility to get a higher resolution with the multiturn measurement. This multi-turn measurement can be made with the 12 bits $100 \mathrm{MHz}$ waveform digitizer which is commercially available. By adding these electronics to a branch of BPM output, we can test the new electronics without any disturbance for the current BPM system. $1 \mu \mathrm{m}$ resolution is expected with this new multi-turn electronics giving less than $2.0 \times 10^{-12} \mathrm{~m}$ geometrical emittance required from ILC-DR.

This extremely small emittance has a big impact to ATF II project, because the beam size with given optics is proportional to square root of the emittance. We might be able to demonstrate a beam size at the focal point smaller than the expected size. This smaller beam size makes ATF II more suitable for the ILC final focus prototype.

\subsection{Options for DR studies in ATF}

Looking over the history of damping ring, Amaldi [63] first proposed the use of damping rings for reducing electron and positron beam emittances. Typically, this is done with wiggler magnets installed in a damping ring. There are two other possibilities, with perhaps one having more of a benefit for future upgrades to the ATF. One possibility was proposed some time ago by Dikansky and Mikhailichenko. They suggested using a linear wiggler system to achieve small emittances at VLEPP [64]. There, they found that $15 \mathrm{GeV}$ electron beams required about $1 \mathrm{~km}$ of wigglers and accelerating structures to decrease emittances the equivalent of a few damping times. Recently, Dugan [65] and Braun, Korostelev, and Zimmermann [66] have revived interest in this technique. As noted by Dugan, 
the length of the bunch train does not determine the length of a linear damping system (LDS). Also, unlike a damping ring, an LDS has no arcs contributing to quantum excitations. Though this option should be pursued as a supplementary damping system for the International Linear Collider, there are space limitations at the ATF that may pose a problem for this technique.

The other, perhaps more viable, option for an ATF upgrade involves using rf wigglers to achieve the needed damping. This technique was explored by Braun et al. [66], because the CLIC damping ring design as it stood did not achieve the desired electron and positron beam emittances. While the goal for normalized rms horizontal/vertical emittances was $450 / 3 \mathrm{~nm}$, the designed damping ring only achieved 578/8.1 nm. Since quantum excitations scale as the square of the wiggler period, the wiggler period is reduced by using this alternative to magnets, thereby providing a real benefit. Rf undulators, which have been considered in the past for synchrotron light sources [67, 68], are defined by the regime where $\lambda_{u} B_{0}<0.01 \mathrm{~T}-\mathrm{m}$, where $\lambda_{u}$ is the undulator period and $B_{0}$ is the peak magnetic field. Braun et al. found that rf wigglers performed better than rf undulators.

The possibilities of installing rf wigglers in the ATF to enhance the damping of the electron beam seems plausible, and a number of studies will be necessary. Among other things, it will be important to investigate the effect of the rf wigglers on the dynamic aperture and the effect on the vertical emittance of the opening angle effect of synchrotron radiation. Braun et al. examined the case of a rectangular waveguide in the $\mathrm{TE}_{10}$ mode; however, more complicated structures, such as disk-loaded waveguides, should be more beneficial and must be investigated.

We propose to optimize an rf wiggler waveguide, design a prototype, and test in the ATF damping ring. Also, we will evaluate the possibility of testing a linear damping system at the ATF if that proves to be feasible. For this case, there are a number of physics and technological challenges that must be addressed. For example, Dugan has pointed out the challenge of implementing high-field, short period magnetic wigglers. We would have to delve deeper into these issues. 


\section{A Proposal of laser facility}

The ATF2 will be an ideal test beds for laser facilities for the ILC as it provides a $30 \mathrm{~nm}$ spot size electron beam with the ILC like bunch structure. The scope of the facility covers R\&D for the photon colliders, electron polarimeters, and polarized positron sources.

\section{A.1 Description of the Project}

In the International Linear Collider, laser-electron interactions will be used for many applications, such as high energy photon generation for the photon collider, electron (and possibly positron) polarimeters, beam size monitors, and polarized positron sources. The photon collider and positron source applications will require high photon fluxes, leading to a requirement of high efficiency for the laser-Compton interaction. Recirculation of the terawatt laser pulses will be desirable to reduce the total average laser power needed by the system [69].

Much of the development of the laser technology can be done without access to an electron beam. However, these systems are unprecedented and a facility where the laser-electron beam interaction can be demonstrated is critical for the final step in the system development. ATF2 is ideal for this facility. The system demonstration requires a beam which is time formatted to match the $337 \mathrm{~ns}$ spacing of the ILC as well as low emmittance so that the beam can be focused to a spot much smaller than the laser focus. ATF2 can fulfill these requirements with a 20 bunch subset of the full ILC bunch train of 2820 bunches. By varying the timing between the laser and the beam we can explore issues involving maintenance of laser quality over the entire bunch train.

Laser systems for the various applications are under development. It is not expected that the ATF2 facility would be needed for laser system demonstrations before 2010. This would allow time for the primary beam physics activities of the facilities to be completed before conversion of the focal point for laser demonstrations.

\section{A.2 High photon flux facilities}

The basic layout of the set up is illustrated in Fig. A.1. The system will be a cut-down version of the system we will need in the ILC. Though it is a cut-down version but needed to be large enough to test issues of the full size/ full power system. The total length of the cavity is desirable to be the bunch spacing of the ATF2 to synchronize the laser pulse with the electron bunches, however it can be shorter if it is cost effective and adequate for demonstrating the technology. The final focus mirror will be about $3 \mathrm{~m}$ away from the focusing point. The system will be enclosed in a vacuum beam pipe so there should be no laser hazard issues. However, it will still need to be enclosed in a clean room environment for construction and maintenance. For the diagnostics downstream of the interaction, a bending magnet is placed to sweep out spent electrons. Since we expect $10^{8}$ photons per 


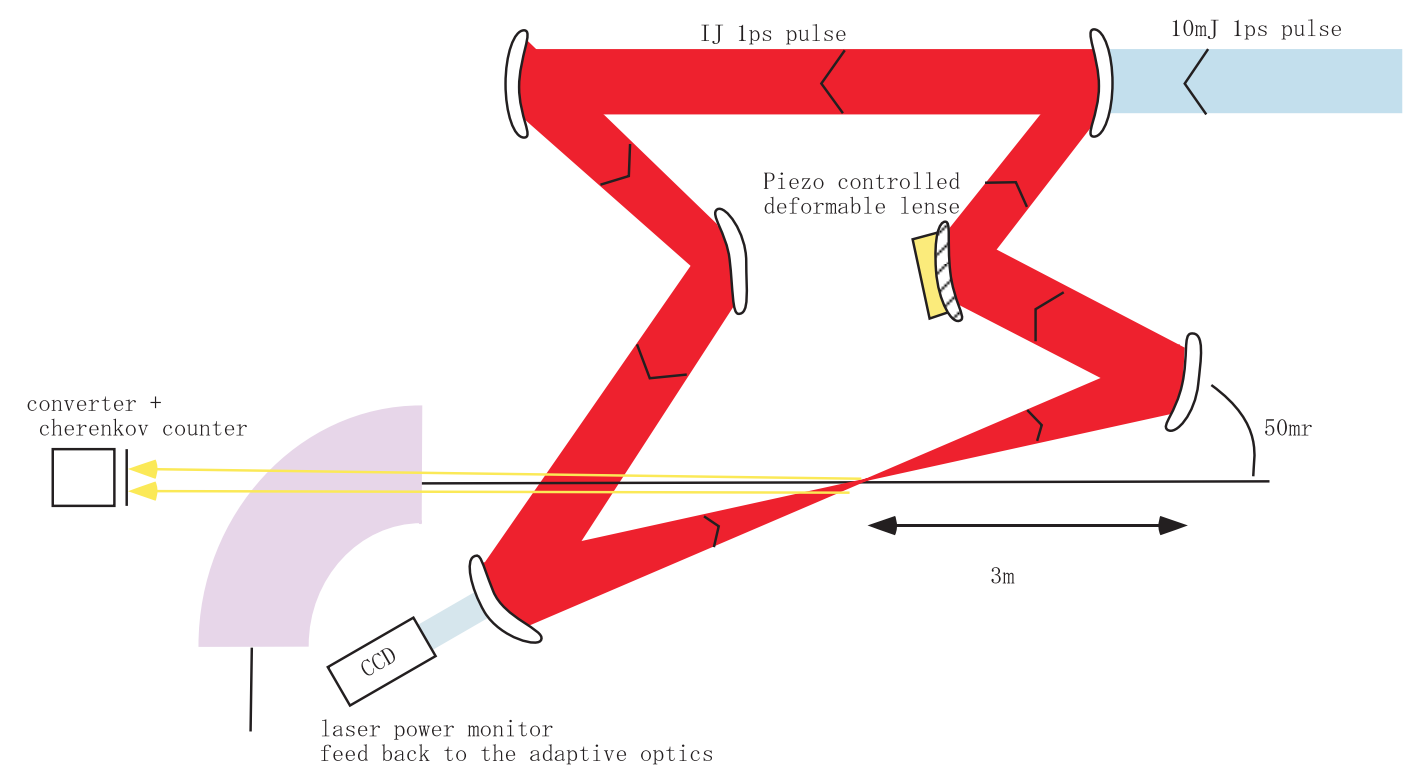

Figure A.1: Schematic Layout of the laser interaction region and recirculating cavities.

laser-electron collision signal should not be a problem, the total number of photon can be measure by a photon detection system which consists of a converter and a Si or a Cherenkov counter. The photon detection system is almost identical to the one used for the experiment for the polarized positron sources [70]. The design and construction of the cavity will be performed by the ILC collaboration with Japanese (KEK, Hiroshima) and US (LLNL) collaborators currently interested is this work.

Fig. A.2 shows the expected photon energy spectrum simulated by CAIN. The electron and the laser parameter for the simulation is summarized in Table A.1. Though electron bunch length of the ATF2 is much longer than the typical ILC parameter, we assumed that the pulse width (and Rayleigh length) of $1 \mathrm{ps}(0.3 \mathrm{~mm})$ which is closed to the realistic parameter for the photon collider. According to the simulation the conversion efficiency is about $3.3 \%$.

Table A.1: Parameters for high intensity photon facility.

\begin{tabular}{lll|lll}
\hline Electron Beam & & & Laser & & \\
\hline Energy & $E$ & $1.3(\mathrm{GeV})$ & Wave length & $\lambda$ & $1.054(\mu \mathrm{m})$ \\
Particle per bunch & $N$ & $1 \times 10^{10}$ & Pulse energy & $A$ & $1(\mathrm{~J})$ \\
Emittance & $\gamma \epsilon_{x} / \gamma \epsilon_{y}$ & $3 / 0.03(\mu \mathrm{m})$ & Pulse Length & $c \sigma_{t}$ & $300(\mu \mathrm{m})$ \\
Bunch length & $\sigma_{z}$ & $9(\mathrm{~mm})$ & Rayleigh length & $Z_{R}$ & $300(\mathrm{~mm})$ \\
IP beam size & $\sigma_{x} / \sigma_{y}$ & $3.4 / 0.035(\mu \mathrm{m})$ & Spot size & $\sigma_{L}$ & $5.0(\mu \mathrm{m})$ \\
IP beta function & $\beta_{x} / \beta_{y}$ & $10 / 0.1(\mathrm{~mm})$ & & & \\
\hline
\end{tabular}

The goal of the facility is to demonstrate the high intensity photon beam using the laser cavity. The $\mathrm{R} \& \mathrm{D}$ issues for the laser cavity fall into three broad areas: 


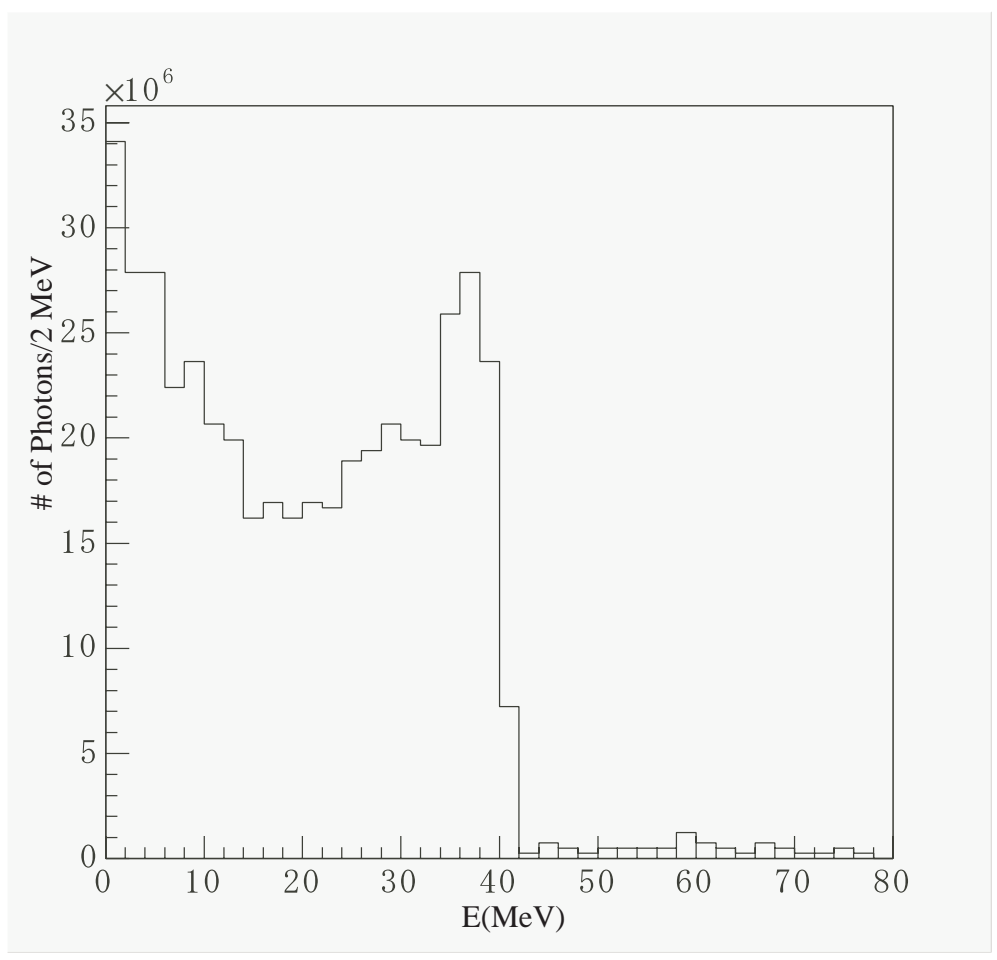

Figure A.2: Expected photon energy spectrum simulated by CAIN.

Stability of the cavity To keep the Q factor of the cavity, the cavity length has to be kept at very high accuracy. It requires a feedback system to control position of the optical components. In addition keeping distance between the focal point and the final focus mirror is crucial to maintain the laser spot size at the conversion point.

Cavity losses. The optics in the system retain $>99.7 \%$ of the laser power in each round trip. This requires the development of high efficiency coating which can survive the high fluence. Final focus mirror may need to be at least $10 \mathrm{~m}$ from the interaction point so that the beam can expand enough to prevent damage to the mirrors.

Wavefront quality. Wavefront distortions will accumulate as the pulse travels through the system. This will limit the size of the beam focus at the interaction point. This can quickly degrade the laser intensity at the focus and reduce the backscattering rate. An adaptive optics system has to be implemented to compensate the distortions. If there are any transmissive optics inside the cavity then non-linear effects may become the limiting factor.

Systems have been created that can do any of these thing individually but a system that does all simultaneously for high power/large scale cavity is unprecedented. The power buildup cavity is popular for the CW laser and power buildup factor of $10^{5}$ is reported and large scale cavity has been developed for the gravitational experiment. The pulse stacking cavities, on the other hand, are not popular as for the CW lasers. A cavity for the laserwire system for the ATF has been reported [71], however, 
feasibility of large scale cavity is yet to be studied. In this project, we envision demonstration of $10 \mathrm{~m}$ scale pulse stacking cavity to prove simultaneous realization of issues as;

- cavity length and wave front stabilization utilize adaptive optics

- feed back system for adaptive optics which is fast enough for 337 ns of 3000 bunches

- high power, $\sim 1 \mathrm{~J} /$ pulse, storage in the cavity

- stable generation of high energy photons.

\section{A.3 Low photon flux facilities}

ATF2 is also suitable for testing the pulse cavity for the electron polarimeters. The laser optics for these applications will typically be much smaller in size but will probably still need to be in a clean room environment. A significant photon beam dump will not be required and this space may be needed for additional diagnostics. There is currently a project to construct a prototype cavity for the polarimeter in Orsay.

\section{A.4 Requirements}

The basic ATF2 facility will produce the electron beam and bring it to a focus. Some additional facilities are required for the laser demonstrations. Space around the electron beam focus must be reserved for the optics that will bring the laser light into collision with the beam. The produced photons will travel along the beam direction and space after the focus will be needed for photon beam diagnostics and a beam dump. A separate beam dump for the spent electrons will be needed as well as bending magnets to sweep the beam out of the photon beamline. Some applications like polarimeters and beam size monitors will have a low rate of Compton backscattering. They will have low power photon beams and largely undisrupted electron beams. Applications like positron source and photon collider will have high power photon beams with spent electrons of large energy spread. The layout of the post-Compton scattering beam lines must handle both of these conditions. 


\section{B BINP kicker design proposal}

In this chapter, we discuss a concept of providing extraction of ILC-like train, with 300 ns between bunches, from the ATF damping ring. One need to stress that while this suggestion is conceptual, and not all details have been worked out yet, it could also be applied to the ILC Damping Ring.

\section{B.1 Low aperture extraction kicker}

Extraction from ATF and formation of ILC-like bunch train, within the framework of the already taken strategic solutions in ATF, appears to be a complex and expensive task. Realization of this task is at the limit of the technical capabilities of the element base produced at present time in the world. In order to solve this task and build a very reliable system, it seems appropriate to adequately divide the appearing difficulties with the adjacent systems, and consider the feasibility of installation of some additional devices.
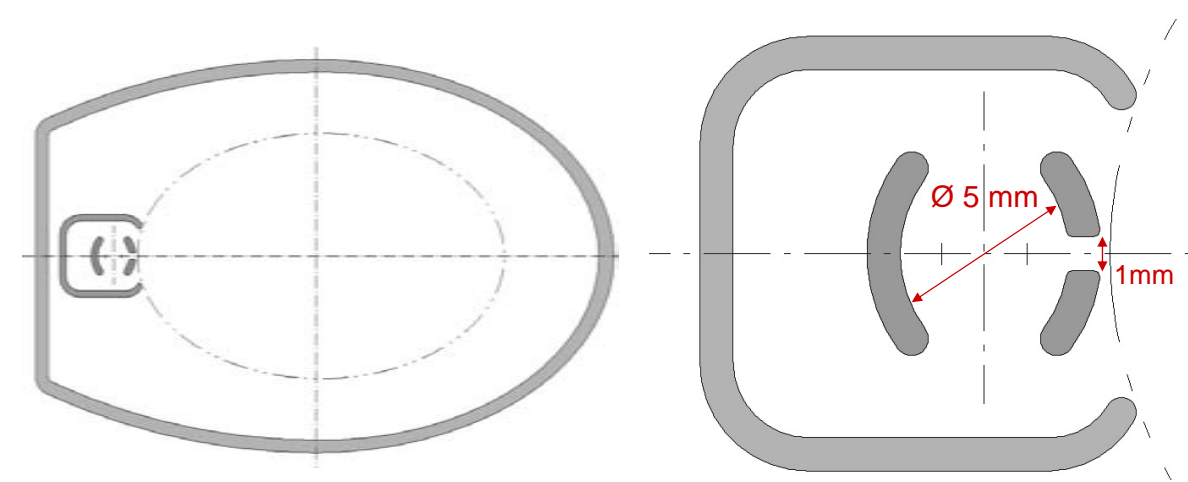

Figure B.1: Cross-section of the vacuum chamber with built-in low aperture kicker (left) and close view of the kicker part with dimensions (right).

Basic problems in accomplishment of this task are connected, first of all, with the need to form the packet of powerful high-voltage pulses with very short rise time (on the order of 1-2 ns) with high repetition frequency (more than $2 \mathrm{MHz}$ ). Very stringent requirements on temporary and amplitude accuracy and stability of the parameters of pulses are imposed. The absence of the suitable switches for shaping of the kicker pulses is the main obstacle. In principle, the power-supply system can be built on the basis of the ultra-high-speed transistor switches of the firm Behlke; however, the single power of such switches is small. Therefore this system will be bulky, expensive, and it can prove to be insufficient reliable. Nevertheless, there are no other more suitable switches available today at the disposal of the developers of ultra-high-speed high-voltage pulsers. 


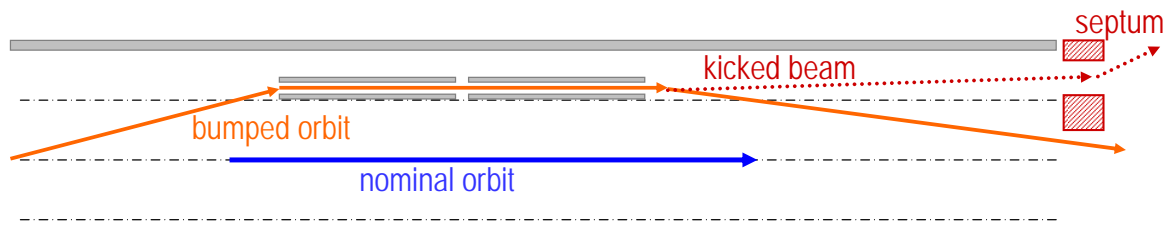

Figure B.2: Longitudinal cross-section of the vacuum chamber with built-in low aperture kicker and schematics of the beam orbits for the nominal and extracted beam.

The technical solution of the problem of extraction from ATF DR and the formation of ILC-like bunch train can be significantly simplified, and the necessary quantity in the power-supply system of kicker's of ultra-high-speed switches is reduced by one - two orders, if we select the strategy presented below. At the same time, it could be proven possible to reduce the quantity of the kicker modules and all the associated elements, thus to lower the cost and increase the reliability of the system as a whole.

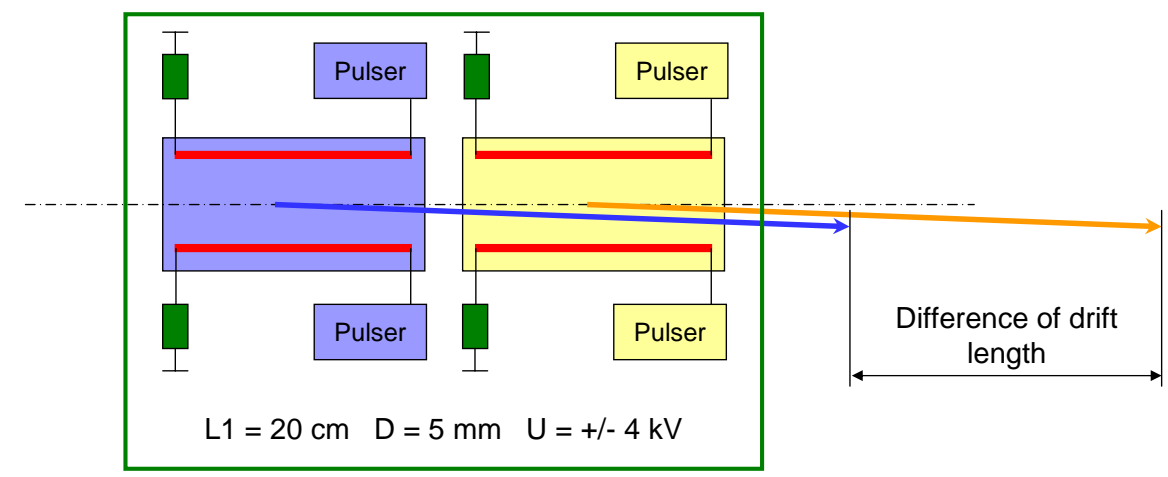

Figure B.3: Two groups of kickers, working on odd and even pulses, allow halving the repetition rate of the switches. The difference of the drift length can be corrected downstream.

The basis of the proposed solution is the fact that the transverse beam size before the extraction is very small. Therefore, from one side, there is no need to form the field of the kicker in the entire geometric aperture of the vacuum chamber. And, from the other side, there is no need to ensure the full-aperture throw of the beam only due to the impact of the kicker. Taking this into account, the basic idea of the proposal can be divided into four parts.

1. Use low-aperture kicker located at the edge of the vacuum chamber as shown in Fig. B.1.

2. Use local orbit bump which slowly drives the beam into the kicker and closer to septum at the end of the damping cycle before the extraction as shown in Fig. B.2.

3. Use two groups of kickers to halve the rep rate of individual kickers, as shown in Fig. B.3. 
4. Correct the drift length oscillation with RF corrector.

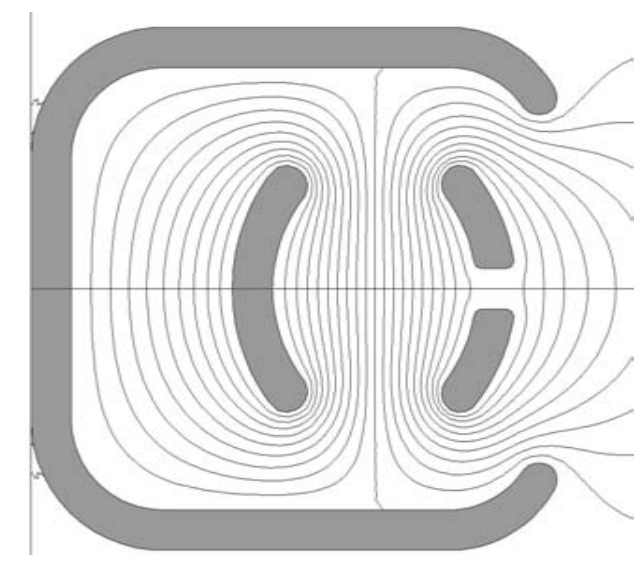

Figure B.4: Calculated field in the low aperture kicker.

Here we assume that even optimistically, Behlke switches could not work with repetition rate needed for ATF extraction. A possible solution: the ensemble of kickers will consist of two groups; moreover, the modules of kickers from the different groups are located alternatively. Each module is fed from its own pulsed power supply with a repetition frequency of about $1 \mathrm{MHz}$, and the entire system works with twice higher frequency.

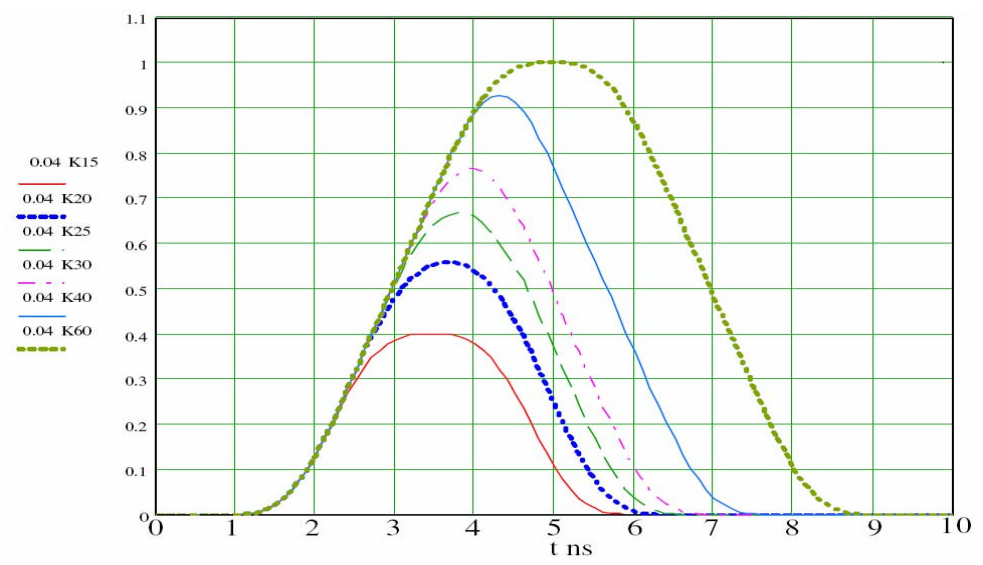

Figure B.5: Kicker pulse shape with fixed amplitude of the traveling wave pulse and for various length of the kicker $(15,20,25,30,40,60 \mathrm{~cm})$. Calculated for quasi-square pulse with $2.5 \mathrm{~ns}$ FWHM duration and with raise/fall (with $\sin ^{2}$ shape) duration of $1.5 \mathrm{~ns}$. This picture shows that the length of the kicker should not be longer than $20 \mathrm{~cm}$.

In this geometry the effective center of the kick become alternately shifted for the adjacent bunches by the length of one kicker module. Accordingly, the drift length will oscillate for the different bunches. 
If this difference exceeds the allowed value, the situation can be mitigated with the aid of the RFcorrector in the extraction line (or special power supply of one of the kickers).

Details of the kicker geometry and calculated fields are shown in Fig. B.4. Dimensions of the plates and of the bus of the kicker were chosen to provide $50 \mathrm{Ohm}$ impedance. The calculated pulse shape for this kicker is shown in Fig. B.5, which demonstrates that in order to have the pulse length less than $6 \mathrm{~ns}$, the length of the individual kicker module has to be $20 \mathrm{~cm}$ or less.

The table of tentative parameters for the kickers is shown in Table B.1. Note that the amount of kick required, depends on the possibility to modify the ATF septum. The present ATF septum has $22 \mathrm{~mm}$ knife thickness. The needed kick is primarily defined by the septum knife thickness. With the drift length of about $4 \mathrm{~m}$, the needed kick is about $5 \mathrm{mrad}$ and the number of kicker modules is about 20 , which would require several meters of space, not available in the ATF ring. As we see, the low aperture kicker idea cannot really be used unless the required kick could be reduced.

Table B.1: Tentative parameters of the low aperture kicker for the ATF2. ${ }^{*}$ The number of modules depends on the possibility to provide orbit correction and modify the septum.

\begin{tabular}{ll}
\hline \hline Energy, GeV & 1.3 \\
Beam size before ejection, mm & $\sim 0.07 \times 0.01$ \\
Kicker raise time, ns & 2.3 \\
Jitter, ns & 0.2 \\
Horizontal kicker aperture $\Phi, \mathrm{mm}$ & 5 \\
Vertical slot in the kicker plate, mm & 1 \\
Kicker plate impedance, Ohm & 50 \\
Amplitude of pulses, kV & \pm 4 \\
Field quality @ beam size, $\%$ & $<1$ \\
Kick stability, \% & $<0.5$ \\
Length of single kicker module, cm & $\sim 20$ \\
Angle due to single kicker module, mrad & $\sim 0.5$ \\
Number of kicker modules & $\mathrm{TBD}^{*}$ \\
\hline \hline
\end{tabular}

Reduction of the required kick primarily depends on the possibility to reduce the septum knife thickness. However, narrower (3-4 mm) knife septum should be possible. For example, septum of SR source Siberia-2 (made by BINP for Kurchatov Institute) has field of septum $2 \mathrm{~S}$, knife thickness about $2.5 \mathrm{~mm}$, aperture $10^{*} 14 \mathrm{~mm}^{2}$, powered by sin-half-wave with duration about $100 \mathrm{~ms}$. The beam size is about $1 \mathrm{~mm}$, the nominal orbit is located about $3 \mathrm{~mm}$ from the wall of the knife. The septum knife is copper with magnetic screen. This example shows that septum with much smaller knife can be constructed. If the septum could be rebuilt, the required kick will be reduced and the low aperture kicker idea could be used for ATF2.

In conclusion we would like to stress that the proposed concept can make it possible to form the ILC-like bunch train at ATF on the basis of contemporary element base with the reasonable cost and 
good reliability, but there is a number of questions still to be answered. First of all, one need to evaluate the possibility to modify septum. The second biggest concern is the effect of wake-fields from the low aperture gaps on the ATF beam. This has been estimated by Karl Bane and described in the next section.

Finally, one need to note that it may turn out that realization of this proposal would require too much modifications of the existing ATF hardware. Still, it may be useful to consider use of this suggestion for the ILC Damping Ring.

\section{B.2 Wakes due to Extraction Kicker}

In the low aperture extraction kicker the beam comes in close approach to metal before being extracted. The question we study here is, What are the wakefields and what is their effect on the beam emittance?

A sketch of the kicker is shown in Fig. B.1. Displaced horizontally toward the outside of the beam pipe is a $200 \mathrm{~mm}$-long metal tube with square cross-section (with $\sim 12 \mathrm{~mm}$ on a side) where one side - the one pointing toward the center of the beam pipe - is missing. Inside the tube are two vertically aligned electrodes separated by $5 \mathrm{~mm}$. The electrode nearer to the center of the beam pipe contains a $1 \mathrm{~mm}$-high longitudinal slot, that allows the beam to pass into the region between the electrodes, in preparation to extraction. The metal tube/electrode combination is split in two equal ( $100 \mathrm{~mm}$-long) parts by a small (transverse) gap.

In wakefield calculations we can ignore the effect of the three-sided metal pipe (the metal is too far from the beam and largely shielded by the electrodes) and also the outside electrode (it affects the beam mainly in the horizontal direction). It is the longitudinal slot in the inside electrode that will have the dominant effect. If the beam finds itself vertically off-center in this slot on any turn, wakefields will be generated that kick the beam (vertically) differentially along the bunch, and thus increase the vertical emittance. Two kinds of wakefields are of concern here: the resistive wall wakefield of the electrode proper, and the geometric wakefield generated at the (4) ends of the electrode. The total effect is approximately given by the sum of the two contributions.

We begin with analytical calculations assuming a simplified model of the problem. Let us model the electrode gap of $1 \mathrm{~mm}$ height by a round collimator of radius $a=0.5 \mathrm{~mm}$ in a beam pipe of radius $b \gg a$. For a gaussian bunch, the shape of the resistive wall (rw) wakefield is given by $f\left(s / \sigma_{z}\right)$ with $f(x)=\int_{0}^{\infty} d x^{\prime} e^{-\frac{1}{2}\left(x-x^{\prime}\right)^{2}} / \sqrt{x^{\prime}}$, with $s$ position within the bunch (negative $s$ points toward the head) and $\sigma_{z}$ is bunch length (see A. Chao's book). The rms of the rw wake is given, in $[\mathrm{V} / \mathrm{C} / \mathrm{m}]$, by

$$
\left(W_{r w}\right)_{r m s}=(0.052) \frac{c L}{a^{3}} \sqrt{\frac{Z_{0}}{\sigma \sigma_{z}}},
$$

with $c$ the speed of light, $L$ the length of pipe, $Z_{0}=377 \Omega$, and $\sigma$ the conductivity of the metal walls.

The rms of the geometric ( $\mathrm{g}$ ) wake of a gaussian bunch in our model collimator, for $\sigma_{z} \gtrsim a$, is given by (K. Bane and P. Morton, SLAC-PUB-3983, 1986)

$$
\left(W_{g}\right)_{r m s}=(0.035) \frac{Z_{0} c}{a \sigma_{z}} .
$$


(It is assumed that the collimator length $L \gtrsim 2 a$.) In this parameter regime the geometric wake is resistive in that the shape of the wake is nearly the same as the bunch shape, i.e. gaussian. Once the rms wake is known, the rms kick angle due to a single pass in the gap is given by $\left(\Delta y^{\prime}\right)_{r m s}=$ $y Q W_{r m s} / E$, with $y$ the beam offset from the center line, $Q$ the bunch charge, and $E$ the beam energy. And finally, the fractional emittance increase (if it is small compared to 1) $\Delta \epsilon / \epsilon \approx \frac{1}{2}\left(\Delta y^{\prime}\right)_{r m s}^{2} / \sigma_{y^{\prime}}^{2}$, with $\sigma_{y^{\prime}}$ the angular divergence of the beam.

As parameters in our calculations we take $L=200 \mathrm{~mm}, a=0.5 \mathrm{~mm}, Q=3 \mathrm{nC}, E=1.3 \mathrm{GeV}$, $\sigma_{z}=8 \mathrm{~mm}, \sigma_{y^{\prime}}=1.1 \times 10^{-6}$, and $\sigma=60(\mathrm{~m} \Omega-\mathrm{mm})^{-1}(\mathrm{Cu}$ at room temperature $)$. The analytical models give $\left(W_{r w}\right)_{r m s}=0.7 \mathrm{~V} / \mathrm{pC} / \mathrm{mm}$ and $\left(W_{g}\right)_{r m s}=2.0 \mathrm{~V} / \mathrm{pC} / \mathrm{mm}$. Note that, because of the transverse gap in the kicker, Eq. B.2 needed to be multiplied by 2 .

For the geometric wake a numerical simulation using T3, the 3d time domain module of MAFIA, was also performed (by C.-K. Ng). A transverse view of the model used in the simulation is shown in Fig. B.6. The curvature in the electrode is not in our model, though the result should not be sensitive to it. For the simulation the length of the electrode is $5 \mathrm{~mm}$ (though the result does not depend in this parameter); the beam was displaced $100 \mu \mathrm{m}$ vertically from the symmetry line between the electrode gap. The wakefield shape, as expected, was gaussian (like the bunch shape). The rms wake for the numerical result (again with the factor of 2 added) is $\left(W_{g}\right)_{r m s}=3.7 \mathrm{~V} / \mathrm{pC} / \mathrm{mm}$, which is about twice the analytical approximation. For a beam with a $100 \mu \mathrm{m}$ vertical offset, the relative kicks $\left(\Delta y^{\prime}\right)_{r m s} / \sigma_{y^{\prime}}$ are: for rw 0.15 and for $\mathrm{g}$ (the numerical result) 0.78 . The geometric wake effect dominates and it is not small.

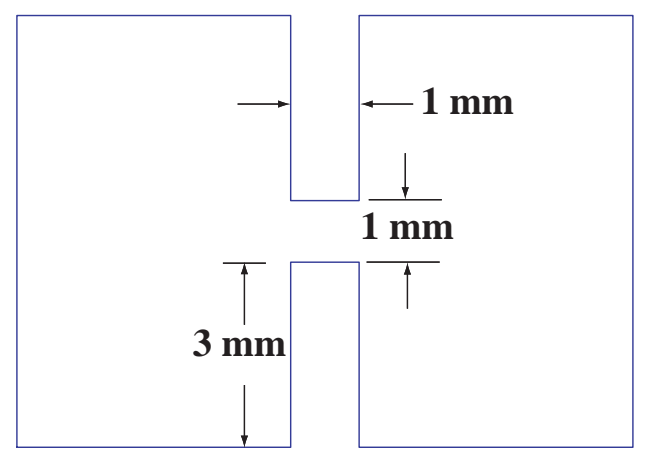

Figure B.6: Geometry used for wakefield calculation.

These calculations have shown that the geometric wakefield of the electrode slot place tight tolerances on the orbit of the beam in the slot. If the beam spends more than one turn within the slot, the wakefield effect multiplies and becomes even larger. Finally, note that since the geometric wakefield dominates, and since it depends on the aperture inversely to the first power, one cannot gain quickly by increasing the aperture of the slot. 


\section{References}

[1] First ILC Workshop Towards an International Design of a Linear Collider, Nov.13-15, KEK, Tsukuba Japan.

[2] K. Kubo et. al., Phys. Rev. Lett. 88194801 (2002).

[3] V. Balakin et. al., Phys. Rev. Lett. 742479 (1995).

[4] P. Tenenbaum, ATF2 workshop, SLAC, January 5, 2005, http://www-conf.slac.stanford.edu/mdi/ATF2.htm

[5] P. Raimondi and A. Seryi, Phys. Rev. Lett. 863779 (2001).

[6] Y. Honda et. al., PRST, 6092802 (2003).

[7] Goals of ATF2 project were first discussed at First Mini-Workshop on International ATF2 proposal Final Focus and Photon Facilities at KEK ATF for the International Linear Collider SLAC, Menlo Park CA-94025, US, January 5, 2005. In particular see contribution from A.Seryi, S. Kuroda, M. Pivi and D.Angal-Kalinin. http://www-conf.slac.stanford.edu/mdi/ATF2.htm

[8] T. Shintake, NIM A311 455 (1992).

[9] S. Kuroda, J Urakawa, H. Hayano, T. Okugi, S. Araki, N. Toge, T. Matsuda ad T. Tauchi A plan of KEK-ATF Final Focus Test Beam Line (ATF2) in the Proceedings of the 26th Advanced ICFA Beam Dynamics Workshop on Nanometre-Size Colliding Beams, September 2-6, 2002, Lausanne, Switzerland.

[10] M. Pivi, A. Seryi, D. Angal-Kalinin, et al., in preparation.

[11] Strategic Accelerator Design (SAD) code homepage: http://acc-physics.kek.jp/SAD/sad.html

[12] R. Assmann, T. Barklow, M. Breidenbach, F.J. Decker, C. Field, L. Hendrickson, D. McCormick, M. Minty, N. Phinney, P.Raimondi, M. Ross, J. Turner, T. Usher, M. Woodley, R. Traller, F. Zimmermann SLC - The End Game, SLAC-PUB-9724.

[13] O. Napoly, et al., FFADA program - Final Focus Automatic Design and Analysis, 1995.

[14] R. Alley et al., Nucl. Instrum. Meth. A379 363 (1996).

[15] H. Sakai et al., Phys. Rev. ST Accel. Beams 5122801 (2002)

[16] K. Balewski et al., Proc PAC 05, in preparation.

[17] P. Tenenbaum and T. Shintake, Ann. Rev. Nucl. Part. Sci. 49125 (1999).

[18] G. A. Blair et al., EUROTeV note, in preparation.

[19] G. A. Blair, CERN-OPEN-2002-057.

[20] P. Tenenbaum and T. Shintake SLAC Pub 8057 (1999) 
[21] T. Shintake; Nanometer Spot Size Monitor using Laser Interferometry. US-CERN-Japan Joint Accelerator School: Topical Course: Frontiers of Accelerator Technology, Maui, Hawaii, 3-9 Nov 1994. In *Maui 1994, Frontiers of accelerator Technology $437-459$.

[22] I. Will, G. Koss, I. Templin NIM A541 467 (2005).

[23] M. Woods, T. Kotseroglou, R. Alley, J. Frisch, A. Hayakawa and T. Shintake FFTB 98-02.

[24] M. Woods, T. Kotseroglou and T. Shintake, FFTB 98-03.

[25] Y. Honda et al., Phys. Rev. Lett. 92054802 (2004).

[26] K.Kubo, ATF Internal Report ATF-99-11.

[27] Marc Ross et al, ATF Report ATF-04-05.

[28] T. Imai et. al., KEK-PREPRINT-2002-16.

[29] Y .Honda, private communication.

[30] P. E. Emma, ATF Internal Report ATF-99-03.

[31] M. D. Woodley and P. E. Emma, "Measurement and correction of cross-plane coupling in transport lines," in Proc. of the 20th Intl. Linac Conference LINAC 2000, SLAC-PUB-8581.

[32] B.I. Grishanov, F.V. Podgorny, J. Rummler, V.D. Shiltsev, Test of Very Fast Kicker for TESLA Damping Ring, Vancouver 1997 Particle Accelerator Conference, pp 237-239.

[33] C. Brooksby et al., A Solid-State Modulator for High Speed Kickers, Chicago 2001 Particle Accelerator Conference, pp 3738-3740.

[34] P.Burrows at al, EUROTEV-REPORT-2005-003-1

[35] Precision Magnet Movers for the Final Focus Test Beam, G. Bowden, G. Bowden, P. Holik, S.R. Wagner (SLAC), G. Heimlinger, R. Settles (Munich, Max Planck Inst.),. SLAC-PUB-6132, SLAC-PUB-95-6132, Jun 1995. Published in Nucl.Instrum.Meth. A368: 579-592, 1996.

[36] R\&D Status of the ATF Damping Ring, Junji Urakawa, et.al, Proceedings of the 4th Workshop on Japan Linear Collider, March 1993.

[37] Vibration Measurements in the KEKB tunnel, M.Masuzawa, et al, Proceedings of8th International Workshop onAccelerator Alignment, November 2004.

[38] The SLC flight simulator was developed by M. Woodley (1993).

[39] P. Emma, "Beam based alignment of sector 1 of the SLC linac," Contributed to 3rd European Particle Accelerator Conf., Berlin, Germany, Mar 24-28, 1992.

[40] C. Hawkes and P. Bambade, "First Order Optical Matching In The Final Focus Section Of The Slac Linear Collider," Nucl. Instr. Meth. A274 27 (1989).

[41] V. Ziemann, "Corrector ironing," SLAC-CN-393. 
[42] R.W. Assmann, P. Raimondi, G. Roy, J. Wenninger, "Emittance Optimization with DispersionFree Steering in LEP," PRST-AB 3:121001 (2000).

[43] N.J. Walker, J. Irwin and M. Woodley, "Global tuning knobs for the SLC final focus," Presented at 1993 Particle Accelerator Conference (PAC 93), Washington, DC, 17-20 May 1993.

[44] P.G. Tenenbaum, "Expanded Studies of Linear Collider Final Focus Systems at the Final Focus Test Beam," SLAC-R-475 (1995).

[45] P. Emma, J. Irwin, N. Phinney, P. Raimondi, N. Toge, N.J. Walker and V. Ziemann, "Beam based alignment of the SLC final focus sextupoles," Presented at 1993 Particle Accelerator Conference (PAC 93), Washington, DC, 17-20 May 1993.

[46] P. Emma, L.J. Hendrickson, P. Raimondi and F. Zimmermann, "Limitations of interaction point spot size tuning at the SLC," Talk given at 17th IEEE Particle Accelerator Conference (PAC 97): Accelerator Science, Technology and Applications, Vancouver, Canada, 12-16 May $199 \%$.

[47] Y. Nosochkov, P. Raimondi, T.O. Raubenheimer, A. Seryi, M. Woodley, "Tuning Knobs for the NLC Final Focus," EPAC'02 Paris (2002).

[48] F. Zimmermann, "Magnet alignment tolerances in the SLC final focus system determined by Lie algebra techniques," Nucl. Instrum. Meth. A364, 231 (1995).

[49] L. Hendrickson, S. Bes, P. Grossberg, D. McCormick, N. Phinney, P. Raimondi and M. Ross, "Luminosity optimization feedback in the SLC," Invited talk at International Conference on Accelerator and Large Experimental Physics Control Systems (ICALEPCS 97), Beijing, China, 3-7 Nov 1997.

[50] T. Lohse and P. Emma, "Linear Fitting Of Bpm Orbits And Lattice Parameters," SLAC-CN-371.

[51] R.W. Assmann, F.J. Decker, L.J. Hendrickson, N. Phinney, R.H. Siemann, K.K. Underwood, M. Woodley, "Beam Based Monitoring of the SLC Linac Optics with a Diagnostic Pulse," PAC97 Vancouver (1997).

[52] F. Zimmermann, P. Emma, "Chromatic and Geometric Spot Size Dilution in the Present SLC Final Focus: Measurement and Simulations," SLAC-CN-405 (1996).

[53] F. Zimmermann et al., "Performance of the 1994/95 SLC Final Focus System," PAC95 Dallas (1995).

[54] F. Zimmermann, P. Emma, N. Phinney, P. Raimondi, T. Raubenheimer, M. Woodley "Overview of SLC Beam Delivery Machine Studies in June/July 1996, and Open Questions," SLAC-CN-406 (1996).

[55] ATF2 optics, http://www.slac.stanford.edu/ mdw/ATF2/

[56] The 1993 IEE Nuclear Science Symposium and Medical Imaging Confreence, volume 1, pages 184 to 187 and 397 to 399.

[57] FFTB Mover, SLAC-PUB-6132. 
[58] ATF Accelerator Test Facility Design and Study Report, KEK Internal 95-4 (1995)

[59] M. Kuriki et al., "Multi-bunch generation by Photo-cathode RF gun",Proceedings of the 14th Symposium on Accelerator Science and Technology at Tsukuba, Japan, 143-145 (2003).

[60] T. Naito et al. "Timing system for Multi-bunch/Multi-train operation at ATF"(in Japanese), Proceedings of Linear accelerator meeting (2000)

[61] S. Sakanaka, ATF internal Report ATF-04-07 (2005)

[62] M. Ross and R. Meller, ATF Report ATF-04-06 (2005)

[63] U. Amaldi Phys. Lett. 61B, 313 (1976).

[64] N. Dikansky and A. Mikhailichenko, EPAC'92, 848 (1992).

[65] G.Dugan, private discussions with a Damping Ring Working Group, also see " Linear Damping Systems for the International Linear Collider," Abstract for PAC'05.

[66] H. Braun, M. Korostelev, and F. Zimmermann, CLIC Note 594 (2004)/CERN- AB-2004-017-ABP and APAC'04.

[67] T. Shintake et al., Proc. Linac Conference, Tsukuba (1982), Japanese Journ. Appl. Phys. 21:L601 (1982), T. Shintake et al., Japanese Journ. Appl. Phys. 22:844 (1983).

[68] M. Seidel DESY-TESLA-FEL-2001-08 (2001).

[69] B. Badelek, et.al, Int. J. of Mod. Phys. A19 5097(2004).

[70] M. Fukuda, et.al, Phy. Rev. Letts. 91, 164801(2003)

[71] Y. Honda, talk given at LCWS2005, March 2005 